\title{
Discovery of New Dwarf Galaxies in the M81 Group
}

\author{
Kristin Chiboucas $^{1}$, Igor D. Karachentsev ${ }^{2}$, and R. Brent Tully ${ }^{1}$ \\ kchib@ifa.hawaii.edu, ikar@luna.sao.ru, tully@ifa.hawaii.edu
}

\begin{abstract}
An order of magnitude more dwarf galaxies are expected to inhabit the Local Group, based on currently accepted galaxy formation models, than have been observed. This discrepancy has been noted in environments ranging from the field to rich clusters. However, no complete census of dwarf galaxies exist in any environment. The discovery of the smallest and faintest dwarfs is hampered by the limitations in detecting such faint and low surface brightness galaxies. An even greater difficulty is establishing distances to or group/cluster membership for such faint galaxies. The M81 group provides an almost unique opportunity for establishing membership for galaxies in a low density region complete to magnitudes as faint as $M_{r^{\prime}}=-10$. With a distance modulus of 27.8 , the tip of the red giant branch just resolves in ground-based surveys. We have surveyed a 65 square degree region around M81 with the CFHT/MegaCam. From these images we have detected 22 new dwarf galaxy candidates. Photometric, morphological, and structural properties are presented for the candidates. The group luminosity function has a faint end slope characterized by the parameter $\alpha=-1.28 \pm 0.06$. We discuss implications of this dwarf galaxy population on cosmological models.
\end{abstract}

Subject headings: galaxy groups: individual M81 - methods: data analysis galaxies: fundamental parameters

\section{Introduction}

Standard $\Lambda$ CDM, hierarchical structure formation models predict an order of magnitude more low mass halos in group environments and as satellites around Milky Way-like galaxies than there are observed dwarf galaxies (Klypin et al. 1999; Moore et al. 1999). The steeper

\footnotetext{
${ }^{1}$ Institute for Astronomy, University of Hawaii, 2680 Woodlawn Dr., Honolulu, HI 96821

${ }^{2}$ Special Astrophysical Observatory (SAO)
} 
slope of the predicted mass function over the observed luminosity function (LF) is the source of the 'missing galaxy' problem. There is growing evidence that there are fewer dwarfs than predicted in both field and cluster environments.

Several explanations to resolve this discrepancy between theory and observations have been proposed. Theoretical solutions include modifying the power spectrum on small scales (Kamionkowski \& Liddle 2000), perhaps by invoking warm dark matter (Colín et al. 2000). Numerical simulations generally predict subhalo counts as a function of total mass or maximum circular velocity. For Local Group satellites, these values are not well determined, making direct comparison between these models and observations difficult unless other parameters, such as the mass within a specific radius, are used (Strigari et al. 2007). However, these more appropriate comparisons still find predictions inconsistent with observations. Number counts can be brought into agreement with models if it is assumed the observed dwarf galaxies reside in large dark matter halos while lower mass dwarfs remain invisible (Stoehr et al. 2002).

A large number of astrophysical solutions have also been put forth to explain the apparent underabundance of dwarf galaxies. One line of reasoning is that models that describe how mass is turned into light are to blame, since these depend on mechanisms such as star formation efficiency and feedback which are intrinsically complex and not well understood. If star formation efficiency is lower in lower density halos, or if large gas reservoirs are expelled from small potential wells after an initial burst of star formation and subsequent supernova activity, low mass galaxies will remain fairly dark and difficult to detect. For very small masses, cooling times may be greater than the age of the universe (Haiman et al. 1996). Destructive processes which act in rich environments such as clusters may decrease dwarf number counts or lower luminosities making them more difficult to detect. Such processes include harassment, strangulation, and ram pressure stripping (Moore et al. 1996; Shava \& Tully 1984; Bekki et al. 2001b; Mori \& Burkert 2000) and would be expected to play a larger role in shaping galaxy populations in denser, more massive, and hotter clusters. Instead, high density regions have been observed to contain a larger fraction of dwarf to giant galaxies than low density regions. Another theory argues that reionization suppresses the collapse of gas in low mass halos thereby producing a shallower faint-end slope in the luminosity function (Thoul \& Weinberg 1996). This will not effect halos that collapsed before the era of reionization - those that resided in denser regions, such as rich clusters, would preferentially collapse earlier when intergalactic gas was still cold and halos could accumulate the gas. Reionization will therefore predominantly suppress baryon accumulation in low mass halos in lower density regions such as the poorer groups and field environment, naturally explaining the trend of lower dwarf-to-giant ratios found in lower density regions (Tully et al. 2002). In support of this theory, Strigari et al. (2007) and Simon \& Geha (2007) find good 
agreement with theoretical expectations if the observed Local Group satellites correspond to the earliest collapsed halos, or the most massive at the time of accretion.

Distinguishing between all these possibilities has proven difficult, but before we can draw any conclusions as to the source of the discrepancy, we must first constrain the number counts of dwarf galaxies and the faint-end slope of the luminosity function. No complete census of dwarf galaxies has been obtained in any environment, although much recent progress has been made in the detection of faint Local Group satellites. With SDSS survey releases, the number of known Milky Way and M31 satellites has nearly doubled in just the past 3 years (Simon \& Geha 2007). This has mitigated the missing galaxy problem, but has not brought the number counts into alignment with $\Lambda$ CDM predictions. Due to the sky coverage required for a complete survey and restrictions caused by the zone of obscuration, a complete census of Local Group dwarf galaxies is not on the horizon.

The determination of the faint-end of the galaxy luminosity function is hampered by the limitations in detecting the very faintest and lowest surface brightness galaxies, and this is compounded by the the great difficulty in determining accurate distances to, or ascertaining group and cluster membership for, such faint objects. To measure the faint-end of the luminosity function for nearby clusters of galaxies, researchers have often resorted to using statistical means to estimate cluster memberships (see e.g. Bernstein et al. (1995); Trentham (1997); Chiboucas \& Mateo (2007)). Others use colors or morphology in an attempt to directly assess membership probability (Secker et al. 1997; Mahdavi et al. 2005). All these methods are fraught with large uncertainty. Spectroscopy can be used to establish membership, but to observe the large numbers of galaxies to the necessary depth is prohibitively time consuming.

The M81 group provides an alternative means to directly establish membership for galaxies to magnitudes as faint as $M_{R} \sim-7$. Residing at a distance of only $3.6 \mathrm{Mpc}$, the galaxies in this group resolve into stars. Supergiants and giants near the tip of the red giant branch (TRGB) resolve over the low surface brightness diffuse late type main sequence population light. Simply the degree of resolution is enough to establish with high confidence the group membership status. From color-magnitude diagrams, one can determine TRGB distances to unambiguously assign membership. Given this fortuitous circumstance, we can probe the faint-end of the LF in this group down to $M_{R} \sim-9$ to high accuracy.

The M81 group is embedded in a filament of galaxies that runs up from behind the Galactic plane, shown in Figure 1. The M81 Group has traditionally been considered to include the galaxies around NGC 2403 and those around NGC 4236, each about $800 \mathrm{kpc}$ removed in projection from M81. Maffei 1-2 and IC 342 are considered to be a separate group, $2 \mathrm{Mpc}$ away in projection. The nearest neighbors at higher supergalactic longitude are a 
group around M101 at $\mathrm{SGL}=64, \mathrm{SGB}=+23$ and the near Canes Venatici group at $\mathrm{SGL}=76$, $\mathrm{SGB}=+9$. It has been shown by Karachentsev et al. (2002a) that the subcomponents of the M81 Group around NGC 2403 and M81 itself are falling toward each other, consistent with a total mass within the zero velocity surface bounding the group of $1.6 \times 10^{12} M_{\odot}$. The interest of the present study is the more dynamically evolved subcomponent around M81 contained within the box in Fig. 1,

We surveyed a $8 \times 8$ degree area centered on the M81 group at $\alpha=10: 05: 00 \delta=$ 68:15:00 J2000 to search for new dwarf members of the group. Apart from the five brightest systems: NGC 2976, M81, M82, NGC 3077, and IC 2574, the inspected area contains 13 previously known dwarf galaxies which were discovered in the 1960-80s on POSS-I prints and the Tautenburg Schmidt telescope plates: Holm I, BK3N, KDG 61, Holm IX, Anon 0952 (Arp's loop), Garland, BK5N, KDG 63, KDG 64, HS 117, DDO 78, DDO 82, and BK6N (van den Bergh 1966; Karachentseva 1968; Boerngen \& Karachentseva 1982, 1985). Later, 4 low surface brightness dwarfs: F8D1, FM1, KK 77, and IKN were found on POSSII films by Caldwell et al. (1998). Froebrich \& Meusinger (2000), Karachentsev et al. (2000), and Karachentsev et al. (2004).

Here we report on the discovery of 22 new M81 group galaxy candidates. In Section 2 we present the observations and data reduction for our survey along with a discussion of the dwarf galaxy detection techniques, survey limits, and follow-up observations. We provide measurements of the properties of the newly discovered dwarf candidates in Section 3.1 and construct the total group LF in Section 3.2. We discuss the dwarf population characteristics

in Section 4.1, the distribution of M81 satellites in Section 4.2, and implications of our survey results for cosmological models in Section 4.3. Conclusions are presented in Section 5. Throughout this work we assume a distance modulus to M81 of 27.80 (3.6 Mpc).

\section{Observations and Data Reduction}

\subsection{Initial survey}

The M81 group was chosen in large part because it is near enough at 3.6 Mpc that stars near the tip of the red giant branch just resolve in ground-based images, enabling derivation of the group luminosity function complete down to very faint magnitudes. Stars in Local Group galaxies resolve down to fainter magnitudes but a complete survey would require allsky coverage. For a comparable survey of dwarf satellites around M31 alone, imaging of over 800 square degrees would be required, including observations within the zone of obscuration. The scale on which dwarfs cluster around parent galaxies is found to depend on the mass 
of the parent halo according to the relation $r_{2 t}=193\left(M_{12}\right)^{1 / 3} \mathrm{kpc}$ (Trentham et al. 2006) where $M_{12}$ is the mass of the halo in units of $10^{12} M_{\odot}$ and $r_{2 t}$ is the second turnaround radius of spherical collapse, similar to the overdensity radius $r_{200}$. At a distance of $3.6 \mathrm{Mpc}$ and halo mass of $1.6 \times 10^{12} M_{\odot}$ (Karachentsev et al. 2002a), M81 has a second turnaround radius of $230 \mathrm{kpc}$, or only 3.6 degrees. A survey of this group with complete areal coverage is therefore made possible with large format CCD detectors such as the square degree MegaCam detector mounted on the 3.6m Canada-France-Hawaii telescope. In addition, the M81 group is interesting in its own right as a rich group with a large late-type dwarf galaxy population, unlike our own well studied dSph-rich Local Group.

The MegaCam is a wide field camera composed of a mosaic of 36 CCDs that cover a total area of $0.96 \times 0.94 \mathrm{deg}$ with chip gaps 13 and 80 arcsec wide. Each CCD is composed of $2048 \times 4612$ pixels of $0.187^{\prime \prime}$. We imaged a 65 square degree region centered around M81 in a raster pattern that resulted in each location being observed twice for a total integration time of 1096s. The survey was conducted entirely in the $r^{\prime}$-band because we were primarily interested in the resolved giant population. Typical seeing was 0.75 arcsec, but ranged between 0.6 and 0.9 arcsec (Figure 2). Most of the data were observed on photometric nights, although a few fields were imaged through thin cirrus. Observations were carried out by CFHT staff in queue mode over 3 semesters in the period 2005B-2006.

\subsection{MegaCam data processing methods}

The raw images were initially processed by TERAPIX, an astronomical data reduction center located at the Institut d'Astrophysique in Paris, which provides pipeline data reduction for MegaCam. The TERAPIX pipeline first evaluates data for image quality, identifies chip defects and cosmic rays, and generates weight and flag-map images. Each image is warped onto a common astronomical projection (TAN) and coordinate system (J2000) using SWarp software and an astrometric reference catalog. SExtractor is run on all images to perform the object detection and photometry. The astrometric and photometric calibrations are determined using SCAMP. Astrometry is performed by downloading reference astrometic catalogs e.g. USNO-B1 (Monet et al. 2003). These are cross correlated with each exposure and a pattern matching algorithm is run. Final astrometric uncertainties are dominated by errors in the reference catalogs. On large scales, the astrometric accuracy is expected to be better than $\sim 0.3$ arcsec. Photometric calibration uses standard stars observed on photometric nights. The weighted, quadratic sum of SExtractor parameter MAG_AUTO differences from overlapping detections is used to put all fields onto a common zero-point scale. Residuals for bright objects are typically less than 0.05 magnitudes rms with even 
smaller zero-point uncertainties.

Our fields were mosaicked in such a way that every region was imaged twice with some additional overlap along the sides of each field. These pairs of $548 \mathrm{sec}$ images had still to be stacked. Because MegaCam images are huge, we first split each image and its associated mask image into quadrants. The mask image contained flagged chip defects such as bad columns and pixels as well as cosmic rays. Images were combined using IRAF tasks for image registration and image combination. Pixels that were flagged in each image were ignored during the average operation. Two to four individual images were used to produce final stacked quadrant images of size $30 \times 30$ arcmin.

SExtractor was subsequently re-run on these combined images. Weight images were created during the stacking step with the weight of each pixel set to the square of the number of image pixels averaged together for the final pixel value. This weight image was used during the SExtractor detection of objects to put a significantly lower weight on those regions such as chip gaps where data came from only a single image as opposed to at least two images as is the case over the majority of the field. This kept SExtractor from finding excess detections in the higher noise level regions. SExtractor parameters were tuned to go deep enough to recover most of the mottles in the known resolved M81 group galaxies by setting detection and deblending levels to quite low values. Object detection was set to $1 \sigma$ with a minimum area of 3 contiguous pixels while the object deblending parameter NTHRESH was set to $1 \sigma$ and DEBLEND_MINCONT to 5E-7. This therefore also had the side effect of inducing SExtractor to recover excess noise, primarily in the halos of bright stars and galaxies, and occasionally in the numerous galactic cirrus patches obscuring this part of the sky.

We checked the reliability of the photometry by comparison with stars of known magnitudes. Perelmuter \& Racine (1995) provide a catalog of $B, V, R$ magnitudes for all objects in the vicinity of M81. We transform the $R$ magnitudes to the sdss $r^{\prime}$ system using the rough transformation $r^{\prime}=\mathrm{V}-0.84(\mathrm{~V}-\mathrm{R})+0.13$ provided in Fukugita et al. (1996a). The average difference in $r^{\prime}$ (calculated - SExtractor measured) for stars with $17<r^{\prime}<19.5$ is 0.01 with $\sigma=0.11$ (Figure 3). Since this is only an approximate color transformation, we take this as strong support for the reliability of the photometry.

\subsection{Detection of 22 new candidate M81 companions}

The tip of the red giant branch lies at $M_{I}=-4.05$, with $(V-I)_{t i p} \sim 1.5$. For $(V-R)_{t i p} \sim 0.8$ and using color transformations for metal poor population II stars from 
Jordi et al. (2006), we find $M_{r^{\prime} t i p} \sim-3.1 \pm 0.1$. With our 18 minute combined exposures, we reach limiting magnitudes for resolved stars of $r^{\prime} \sim 25.0-25.5$. From Schlegel et al. (1998) maps, galactic reddening $A_{r^{\prime}}$ in this region is typically 0.1-0.2 but as high as 0.7 magnitudes. Because the M81 group is so nearby at a distance of only 3.6 Mpc $\left(\langle m-M\rangle_{\circ}=27.8\right)$, we can resolve stars down to limiting magnitudes of $M_{r^{\prime}} \sim-2.9 \pm 0.5$. The radial distance within the group will also affect the limiting absolute magnitude by \pm 0.15 . Thus, stars at the tip of the red giant branch are just at the edge of detection in our MegaCam images while young main sequence and red supergiant stars along with intermediate age AGB stars are well resolved.

Two different methods were used to search the MegaCam images for new M81 group dwarf galaxy candidates. The first method used visual inspection of the images. Final images were examined by eye by two of the authors (IK and KC) for new candidates by identifying objects that had resolved and semi-resolved faint stars. Good candidates also had an associated diffuse low surface brightness (LSB) component, although this was not observed in every case. 17 new candidate M81 dwarf members were detected in this way.

The second method used to discover new candidate dwarfs implemented a 2-point correlation auto-detection routine. The auto-detection code calculates the 2-point correlation using the natural estimator for objects having magnitudes $22<r^{\prime}<27$ on scales of 1.1-6.4 $\operatorname{arcsec}(0.02-0.11 \mathrm{kpc}$ at the distance of M81) within boxes of size 200pix. The code is set up to use any box or scale size but we were focusing on smaller galaxies with a concentration of resolved stars. Presumably large diffuse fields of stars could be missed, although the code did pick up a number of these as well. As the box marches across the image, the correlation values within the box are summed and any region with an excess value is flagged. This value was empirically chosen to be 80 based on recovery of previously known and newly visually detected galaxies, and based on tests of the recovery of artificial galaxies (see below). The value chosen maximized the ratio of the rate of recovery of artificially added galaxies to noise detections, but does then necessarily impose minimum magnitude and concentration limits on our survey. All regions that were flagged were checked by eye. The majority turned out to be noise detections along still remaining bad columns, or an excess of noise spikes around halos of bright stars and galaxies or within cirrus. Another large subset of the detections were distant galaxy clusters. The remainder include the M81 candidates.

The code recovered all previously known dwarfs and most of the ones found by eye in this paper, the 3 exceptions being d0957+70, d0944+69, and d1016+69. For these 3, too few resolved points were detected by SExtractor. It did pick out 2 dwarfs, d1009+70 and d0946+68, which have more of a diffuse LSB component than a resolved stellar population and are thus more likely to be more distant objects. 
The code also found a number of candidates not picked out by eye. A couple of these appear to have both stellar and diffuse LSB components: $\mathrm{d} 1006+67$ is the best of these candidates with a resolved stellar component contained within a diffuse low surface brightness region. d0934+70 is hidden behind cirrus but appears to have both a faint resolved stellar component and excess diffuse emission visible over the cirrus. d1048+70 appears to have a diffuse component but only a semi-resolved stellar component. If real, this may perhaps be a more distant dwarf. A couple more have an excess of faint stellar-like detections but in these cases the objects do not appear to be concentrated nor have an LSB component and may prove to be distant galaxy clusters or chance concentrations of stars. Coordinates and basic parameters for a total of 22 new candidate dwarf galaxy group members are listed in Table 1.

We display thumbnails of 3 candidate M81 group blue compact dwarfs (BCDs) in Figure 4 along with a mosaic of another 9 good dwarf member candidates in Figure 5, Each galaxy image is 1.5 arcmin on a side. In Figures 6- 7 we display candidates which may turn out to lie slightly in the background of the M81 group or simply be artifacts in the data, foreground clumps of stars, or distant galaxy clusters. Figure 8 displays a map of our 60 square degree survey region and the distribution of the new candidate sources along with previously known group member galaxies.

In Figure 9, we display histograms of the number counts, $\mathrm{N}\left(m_{R}\right)$, of the SExtractor detections within a radius of 30 arcsec centered on each of the new M81 candidates. For the 3 BCDs and d0944+71, another bright candidate, we construct histograms for detections out to a radius of 45 arcsec. A rough estimate of the fore/background object counts is obtained by measuring the number counts in a same sized region 2.5 arcmin west of each galaxy. These are displayed as shaded histograms overlaying the candidate population histograms.

\subsection{Limits of our Detection Methods}

We tested our automated method of detecting dwarf candidates through the 2-point correlation of resolved stellar populations using false stars. We wish to determine to what limiting stellar densities and magnitudes our galaxy detection routines are sensitive. We add false stars to images using the IRAF package ARTDATA and convolve them with the seeing of the field. To simulate false dwarf galaxies, large numbers of stars are added to real images distributed according to an exponential spatial density profile with an effective radius, $\mathrm{r}_{e}$, ranging from $4-90 \operatorname{arcsec}(0.07-1.6 \mathrm{kpc})$. We add 100-120,000 stars to each galaxy realization ranging in magnitude from $r^{\prime} \sim 30.0$ at the faint end to $24.7-25.3$ at the bright end. We take the tip of the RBG magnitude as our bright limit (24.7) but include up to 
0.6 magnitudes of extinction. We also include a number of simulations with a bright limit up to 22. This allows us to simulate galaxies with a range of stellar populations, from those having entirely old stellar populations to those also containing a young, bright component.

The stellar luminosity function has the form of a power law with power $\sim 0.3$ for the RGB, as measured from CMDs in our previous work. We allow this value to range randomly between $0.25-0.35$ in the simulations. We use the STARLIST task to generate a file suitable for input to MKOBJECTS. Forty false galaxies were added at a time to the MegaCam images. Simulated stars were first convolved with the seeing size and poisson noise was added before objects were added to the real images. A total of 1900 galaxies were generated in this manner. In Figure 10, we display a few examples of our simulated galaxies. SExtractor was re-run on these images and the 2-point correlation automated method was then used to search for these artificial dwarf galaxies.

Although we expect the detectability of a galaxy to depend only on whether the dwarf galaxy has a significant resolved stellar population, we find that the detection rate and limiting depth of our survey depends on a number of factors. The primary factors are the total number of resolved stars and the concentration of these stars. These correspond to the typical measured quantities of total integrated magnitude and surface brightness of the galaxy. Results of 1200 artificial galaxy realizations are displayed in Figure 11, We plot the integrated total magnitude and half-light radius for each simulated galaxy. Open points are those objects which landed on single exposure chip gap regions of the MegaCam field, or fell on bright stars or galaxies and are less likely to be recovered. Large black diamonds represent real candidate dwarf galaxies discovered in this survey. The dashed line displays the magnitude at which recovery drops to $90 \%$ within 2-magnitude bins, while the solid line represents the $50 \%$ completeness limit. We find that we are able to detect compact galaxies with $\mathrm{R}_{e}<11$ arcsec down to $r^{\prime}=20.5$, or about $M_{r^{\prime}}=-7.3$. For galaxies with $\mathrm{R}_{e}<30$ arcsec, we are able to reach $r^{\prime}=19.0(-8.8)$ with at least $50 \%$ completeness. For galaxies larger than $\mathrm{R}_{e}=30 \operatorname{arcsec}(\sim 0.5 \mathrm{kpc})$ we are at least $50 \%$ complete to $r^{\prime}=18(-9.8)$. Assuming real dwarf galaxy sizes of $\mathrm{R}_{e}<1 \mathrm{kpc}$, we expect our survey is $90 \%$ complete down to about $r^{\prime}=18.0$, or about -9.8 at the distance of M81.

In Figure 12, we display the survey detection limits in the mag $-\mu$ plane. Symbols are as before. Lines of constant effective radius are labelled. A curved line is drawn that best bounds the region within which simulated galaxies are recovered. This is the traditional curve that describes isophotal limits for exponential profile galaxies (Allen \& Shu 1979). The completeness of this survey depends on other factors, but the recovery boundary is adequately described by isophotal limits. At a given magnitude, as one goes to fainter surface brightesses, recovery fails because of increasingly low concentrations of stars and a 
failure of the eye or 2-point correlation methods to distinguish these from the background. At a given surface brightness, as one goes to fainter magnitudes, objects become smaller with decreasing stellar population sizes. Therefore, while not necessarily isophotal limits, the detection limits imposed on this survey can be translated to limiting angular sizes and surface brightness.

However, the detection efficiency will also vary by location in these fields. Because our survey just reaches the tip of the RGB, any galaxy with a pure old population is detected only at brighter magnitudes and surface brightnesses in regions of high extinction and fields with worse seeing. We test this using simulated galaxies by generating another 240 galaxies with 0.5 magnitudes of extinction. See Figure 13, On average, the $50 \%$ limit in the recovery is 0.3 magnitudes brighter than for the sample added with $0-0.2$ magnitudes of extinction. Although the number statistics in this sample is small, this suggests that the completeness limit in magnitude depends directly on the level of extinction and that our completeness limits may vary by as much as 0.6 magnitudes throughout our survey region. Similarly, the depth of the group may affect completeness limits. Variations in the distance with respect to $\mathrm{M} 81$ of $\pm 300 \mathrm{kpc}$ affect apparent magnitudes by \pm 0.18 mag. This is a very small effect but implies that the dwarfs on the near side of the group with pure old populations are more likely to exhibit detectable resolved stellar populations than those on the far side.

We test the effect of poorer seeing by simulating 400 galaxies with 0.9 arcsec seeing, degraded from the earlier tests with $0.64-0.75$ arcsec seeing. Results are presented in Figure 14. The solid line denotes the location where completeness drops to $50 \%$ with 0.9 arcsec seeing, while the dashed line represents the $50 \%$ completeness for seeing no worse than 0.75 arcsec. We find evidence that seeing has an effect on the recovery of resolved dwarfs, and this is most significant for compact galaxies $\left(\mathrm{R}_{e}<20\right.$ arcsec) where the completeness shifts 0.8 magnitudes brightward. This is to be expected. In these cases worse seeing blends the light of these small objects to produce a smooth, less resolved low surface brightness object. With the small number statistics, it is not clear that at larger $\mathrm{R}_{e}$ the 0.2 arcsec worse seeing will affect recovery by the measured 0.4 magnitudes, but the results do indicate that seeing has an affect on the detection rates of these objects. Fortunately, only $\sim 19$ combined images, or $11 \%$ of our survey area, will be affected by such poor seeing.

To a lesser extent, one might expect the bright cirrus patches present throughout much of this region to affect detection limits through increased sky noise. From artificial galaxy tests, however, we do not find this to significantly affect our recovery rates. Regions of high galactic stellar density can also limit detectability for the more diffuse dwarfs. At galactic latitudes ranging between $39-44 \mathrm{deg}$ this is not too much of a problem, but a few fields exhibited non-uniformly distributed excess numbers of stars throughout the images, 
increasing the confusion level. This will primarily affect fainter, lower surface brightness dwarfs with fewer total numbers of resolved stars spread over a larger area.

Finally, two areal coverage issues will affect recovery. Any small dwarf lying behind bright saturated stars or galaxies will likely be missed. From false galaxy tests, where the galaxies are randomly placed within the images, we find less than $4 \%$ of recoverable galaxies remain undetected because they landed on bright objects. This primarily affects only the smallest galaxies which can hide entirely behind brighter foreground obects, although a few more extended simulations were obscured by bright stars or galaxies. Also, due to the survey strategy of observing each field twice by offsetting between images by half a field, all chip gap regions in the MegaCam field are observed for a total of only 10 minutes. With half the exposure time as the rest of the field, we expect the magnitude completeness limits in these regions to be about 0.75 magnitudes brighter than the above described survey limits. Because the detected dwarf galaxies in M81 have angular sizes generally greater than 16 arcsec, the vertical chips gaps of 13 arcsec should not affect recovery of any but the tiniest galaxies if by misfortune they should fall in these regions. There are however two large horizontal chip gaps which have single exposure widths of 80 arcsec each across the length of each MegaCam field, and this gap size is essentially doubled in our mosaic of two images per field. Since all our newly discovered dwarf galaxies have angular extents smaller than 160 arcsec, it is therefore entirely possible for dwarf galaxies to be lost in these regions. The total area of these single exposure regions, including the area at the top and bottom of our full mosaic, is 9.7 square degrees, or $\sim 15 \%$ of the total survey area.

Based on these results, we find that we are nearly $100 \%$ complete to $r^{\prime}=17$ and $90 \%$ to $r^{\prime}=18\left(M_{r^{\prime}}=-9.8\right)$. At fainter magnitudes, a large number of factors act to diminish our ability to detect group galaxies, although the primary factors are simply the limiting magnitude and surface brightness to which we are sensitive. Factors such as worse seeing, higher extinction, areal coverage by brighter objects, and single exposure regions serve to push these limits to slightly brighter magnitudes.

\subsection{Follow-up Spectroscopy}

Follow-up spectroscopic observations were carried out with FOCAS on the 8m Subaru telescope and SCORPIO on the Special Astrophysical Observatory 6m BTA telescope. Spectra were obtained for our three BCD candidates along with several globular clusters in a couple of previously known M81 dwarf galaxies.

Observations with Subaru were made 22-24 Nov 2006 using the R300/mm grating in 
second order and VPH 600-450 grating in first order. A two arcsec slit was used with the VPH grating, and images were binned $4 \times 2$, providing a spectral resolution of $10 \AA$ at $4000 \AA$. For the R300 grating, a 1 arcsec slit and $4 \times 1$ binning was used to obtain a spectral resolution of $10 \AA$. Spectral coverage ranged from $3800-5250 \AA$ for the VPH and $3650-5950 \AA$ for the R300 grating. Exposure times varied from object to object.

Longslit spectra were obtained with FOCAS of globular clusters (GC) in the previously known faint dwarf galaxies F8D1 and IKN. The globular cluster in F8D1 was originally noted as a potential GC in Caldwell et al. (1998). For two bright candidate galaxies (both BCDs) d $1028+70$ and d0958+66, we obtained spectra by simply placing the slit across the long axis of the galaxy. These data were processed using standard IRAF routines. Images were first cosmic ray corrected using CRMEDIAN and then bias subtracted using 10 median combined bias frames. Arcs were observed before or after each observation and used to wavelength calibrate the spectra. Lines in the arcs were identified using IDENTIFY and REIDENTIFY, and FITCOORD was used to obtain a dispersion correction solution which was applied using TRANSFORM. The two-dimensional spectrum for each object in the slit was extracted using APALL. Finally, RVSAO/XCSAO (Kurtz \& Mink 1998) was used to obtain a cross correlation solution from template spectra for the redshift of each object and hence the galaxy itself. Spectra are displayed in Figure 15. Radial velocities are listed in Table 2 .

Further observations were performed for the 3 BCD candidates with SCORPIO in longslit mode installed in the BTA 6-meter telescope prime focus. The grism VPHG400 was used with a 1 arcsec slit yielding the spectral range $\sim 3500-7500 \AA$ chosen to include $\mathrm{H} \alpha$ with a resolution of $3 \AA /$ pixel and FWHM $\sim 20 \AA$ /pixel. The objects d1028+70 and d0958+66 were observed during the night 11 November 2006 with the exposure time of $600 \mathrm{sec}$ under $2^{\prime \prime}$ seeing. The galaxy UGC 5497 (d1012+64) was observed on 20 October 2007 with an exposure time of $900 \mathrm{sec}$ under $2.5^{\prime \prime}$ seeing. Spectrophotometric standard stars were also observed for calibration. The standard pipeline with the use of MIDAS, as described in Afanasiev \& Moiseev (2005), was applied for the reduction of these long-slit spectra. All three BCD candidates show knotty $\mathrm{H} \alpha$ emission. We display the spectra in Figures 16-18. Due to the short exposure times and low $\mathrm{S} / \mathrm{N}$ it is difficult to measure line ratios. However, we note that the [NII] $\lambda 6584$ line is quite weak in comparison to $\mathrm{H} \alpha$, especially for d0958+66. We estimate an upper limit for the line ratio of 0.05 for this galaxy. From the relations of Kewley \& Dopita (2002) and Pettini \& Pagel (2004), this leads to an upper limit for the metallicity of $0.3 \mathrm{Z}_{\odot}$. Mean heliocentric radial velocities of the objects are included in Table 2 . 


\subsection{Multi-band Imaging Follow-up}

Using the UH 2.2m with Tek 2K CCD on 19-24 Dec 2006, we imaged 9 of the dwarf galaxy candidates in $V$ and $I$-bands. Seeing ranged from $0.7-1.4$ arcsec and 5 nights were photometric. Images were $4 \times 900 \mathrm{~s}$ in the $V$-band and $5 \times 720 \mathrm{~s}$ in the $I$-band. Standard stars were observed on each photometric night. Images were processed using standard IRAF routines. Due to the slow speed of the mechanical shutter, a shutter mapping correction was also implemented. This was necessary for the short exposure images (5-20s) of the standard stars.

In most cases, the images did not go deep enough to produce useful color-magnitude diagrams (CMD). For 8 galaxies observed on photometric nights, we measure integrated $V-I$ colors within circular apertures out to the effective radius as measured in the $r^{\prime}$ band (Section 3.1). We correct for extinction using the maps of Schlegel et al. (1998). $V-I$ colors are listed in Table 3. We also generate color images of the 9 candidates. To create $B$-band images we take $2 * V_{\text {image }}-I_{\text {image }}$. The $V$ and $I$-band images are taken to be $\mathrm{G}$ and $\mathrm{R}$ respectively. Color images are displayed in Figure 19.

Large young, blue stellar populations are evident in d0926+70, d0958+66, d0959+68, and d1028+70. These are objects classified by us as BCDs, dIrs, and tidal dwarfs. These multi-band data may not be going deep enough to reach the tip of the red giant branch. The lack of visible resolved stars in d0955+70 suggests it may consist predominantly of an older stellar population. Similarly, if members of the M81 group, d0934+70 and d1006+67 also appear to contain predominantly older stellar populations.

We have recently obtained $\mathrm{HST} / \mathrm{WFPC} 2$ multiband imaging for 14 of the candidates. These data will be deep enough to measure distances from the CMD tip of the red giant branch. Results will be presented in a subsequent paper.

\section{Results}

\subsection{Properties of the M81 companion candidates}

To determine centroids of the new objects, we used the intensity weighted means of pixel values, $\frac{\sum \vec{x} I}{\sum I}$, around each galaxy. Ellipticity and position angle around these centroids were then derived from the intensity weighted second moments (Secker \& Harris 1997). Measuring

total magnitudes for these very faint and diffuse extended resolved galaxies is challenging because of expected contamination from foreground stars, and in several cases, from excess foreground cirrus reflected flux, not to mention extinction. We therefore estimated total 
magnitudes using several methods.

We first mask all obvious bright foreground stars and background galaxies around each of our candidates. Simple aperture magnitudes were obtained for each galaxy with radii 50, 100,150 , or 200 pixels $(9.35,18.7,28.05$, and 37.4 arcsec) depending on the visible extent of the galaxy. The sky was determined in an annulus 4 pixels wide with a radius of 400 pixels. Alternatively, we measure total flux in an identical sized aperture in 4-5 regions around each galaxy. Taking the average of these as an estimate for the contribution from both sky and foreground/background object flux we subtract this from our object flux for a second estimate of total magnitude.

To obtain structural parameter measurements for each galaxy, we extracted fluxes in successive elliptical apertures around each galaxy out to a radius of 40 arcsec and truncated the data if it exhibited a $>30 \%$ increase in slope attributed to contamination by an unmasked neighboring object, or, more often, to features like bright cirrus bands. The central 4 arcsec were also discarded. We then fit both the surface brightness profile with a Sersic function and the curve of growth with a cumulative Sersic function. We extracted parameters from the best of these two fits. This provides a third estimate of the total magnitude along with central surface brightness, effective surface brightness, and half-light radius. The generalized Sersic function is defined as

$$
I(r)=I_{\circ} e^{-\left(r / r_{\circ}\right)^{1 / n}}
$$

where $n$ is the Sersic parameter. For $n=1$, this reduces to an exponential profile which usually provides a good fit for disk and dwarf galaxies, and for $n=4.0$ becomes a de Vaucouleurs profile. The cumulative Sersic function is then

$$
I(r)=2 \pi \sigma_{\circ} r_{\circ}^{2} n \cdot \gamma\left[2 n,\left(r / r_{\circ}\right)^{1 / n}\right]
$$

where $\gamma[\mathrm{a}, \mathrm{x}]$ is the Incomplete Gamma function,

$$
\int_{0}^{x} \exp (-t) t^{a-1} d t
$$

(Jerjen 1995). This can be integrated to obtain the total object flux,

$$
I_{t}=2 \pi \sigma_{o} r_{o}^{2} n \Gamma[2 n]
$$

with total magnitude, 


$$
m_{t}=\mu_{o}-5 \log \left(r_{o}\right)-2.5 \log (n \Gamma[2 n])-2.0
$$

where $\Gamma[a]$ is just the Gamma function.

The fitting of this nonlinear function to the data was done using a Levenberg-Marquard algorithm (Press et al. 1992) which performs a $\chi^{2}$ minimization that implements an inverseHessian method far from the minimum and switches to a steepest decent method as the minimum is approached. In Figures 20 - 25, we display both curve of growth and surface brightness profile fits for our candidate galaxies. From these fits we directly obtained the central surface brightness, scale length, and profile type, where scale length in the generalized Sersic function is directly related to the half-light or effective radius through

$$
R_{\circ}^{1 / n}=R_{e}^{1 / n} /\left(2.3026 b_{n}\right)
$$

with $\mathrm{b}_{n}=0.868 \mathrm{n}-0.142$ (Caon et al. 1993).

The majority of the galaxies are best fit with Sersic index $n<1.0$. From inspection of the plots it is clear that these low $n$ fits are primarily driven by large cores, typical of dwarf galaxies. In the outer radii, the surface brightness profile tends to drop exponentially. We overlay best fit exponential profiles to the outer radii in the surface brightness profiles of Figures 20- 25. A number of cases exhibit a sharper drop-off, but are still consistent with an exponential fall-off within the large uncertainties of the low surface brightnesses at these radii.

We estimate typical magnitude measurement errors of 0.5 . Sources of uncertainty come from sky determination (0.4, estimated from curve of growth fitting when using 3 different estimates of sky values), foreground/background contamination (typically 0.2 , from magnitudes measured with and without masking of likely foreground sources), aperture magnitude measurement error from qphot (0.04), and the zeropoint uncertainty $(0.015$, estimated from comparison with Perelmuter \& Racine (1995) catalog stars). Cirrus may also effect measurements of some of the galaxies. Sky estimates in these regions have an even larger uncertainty, and faint old stellar populations may be lost in the excess sky noise. In particular, d0934+70, if real, appears largely obscured by cirrus. d0944+71, d0959+68, and d1048+70 are strongly effected by fore or background objects. We estimate total magnitude uncertainties of 0.8 for d0958+68 and 0.6 for $\mathrm{d} 1048+70$. The magnitude for $\mathrm{d} 0944+71$ is corrected by assuming a symmetric shape. Flux from the star superposed quadrant is replaced by the flux in the symmetrically opposite quadrant. We assume a magnitude uncertainty of 0.8. Candidate d1019+69 is affected by missing data in a chip gap; the measured magnitude is corrected in 
a similar way as d0944+71.

We estimate measurement errors in $\mathrm{R}_{e}$ by comparing results from the surface brightness profile and curve of growth fits and comparing differences from fits using slightly different estimates for the mean sky value. We find fractional uncertainties in $\mathrm{R}_{e}$ range from $1 \%$ for the bright BCD d1028+70 to $40 \%$ for low surface brightness object d0955+70, with a median uncertainty of $10 \%$. Similarly, we find an uncertainty in the central surface brightness ranging from 0.05 to 0.5 magnitudes $\operatorname{arcsec}^{-2}$ with a median uncertainty of 0.2 .

Because of the shallow nature of our survey, we can assume that our magnitude and size measurements are lower limits. To obtain estimates of total magnitudes, all measured magnitudes had to be corrected for light lost in the sky noise. Since many of the profile fits were influenced by the bright O/B stars, it is possible that measured profiles do not accurately reflect that of the underlying old stellar populations. We therefore calculate the missing fraction of light assuming both the fitted profile shape and an exponential surface brightness profile. Dwarf galaxies may be better fit with King profiles than exponential due to the presence of large cores and possible truncation in the wings of the profile. This is supported by our generalized Sersic function profile fits which find $n<1.0$ for most of our galaxies, implying a steeper fall off of the galaxy light in the wings of the profile. Magnitude corrections calculated by assuming exponential profiles may therefore be overestimates for these galaxies, and thus provide upper limits on the total galaxy luminosities.

From an isophotal limiting surface brightness (ranging from 27.0 to 28.5 depending on the sky level and presence of cirrus), along with the measured central surface brightness and total magnitudes, we extrapolate to obtain the intrinsic total magnitude. Over $x$ scale lengths, $x=r / r_{\circ}$, the surface brightness of an exponential profile drops by

$$
-2.5 \log e^{-x^{1 / n}}=1.086 x
$$

for $n=1$ or

$$
=1.086 x^{1 / n}
$$

for a generalized Sersic profile. The number of scale lengths observed between $\mu_{o}$ and $\mu_{\text {lim }}$ is

$$
\Delta x=\left(\mu_{\text {lim }}-\mu_{o}\right) / 1.086
$$

or

$$
\Delta x=\left(\left(\mu_{l i m}-\mu_{o}\right) / 1.086\right)^{n} .
$$

For $m_{T}-m_{\text {lim }}=m_{\text {extrap }}$ with

$$
m_{i} \propto 2.5 \log \left[(1+x) e^{-x}\right] \quad \text { (exponential) }
$$


and

$$
m_{i} \propto 2.5 \log \left[\int_{0}^{r_{i}} e^{-x^{1 / n}} r d r\right] \quad(\text { Ser sic }),
$$

we find the extrapolation beyond the measured magnitudes is

$$
\Delta m=2.5 \log \left[1-(1+\Delta x) e^{-\Delta x}\right]
$$

and

$$
\Delta m=2.5 \log \left[\gamma\left[2 n, \Delta x^{1 / n}\right] / \Gamma[2 n]\right]
$$

for exponential and generalized Sersic profiles respectively. See also Tully et al. (1996). Exponential profile corrections from the isophotal magnitude are less than -0.1 mag for our brightest new candidates, but as high as -1.2 mag for the faintest objects with a mean value of -0.4 . Corrections for the best fit Sersic profiles range from 0 . to -0.8 magnitudes with a mean of -0.2 . Besides assuming a profile shape, these corrections further assume an old stellar population exists which may be too faint to detect, even as a diffuse low surface brightness component, in some cases. For potential tidal dwarfs like d0959+68, it is not clear that this assumption is valid. There may be evidence that d0959+68 is dominated by a very young population formed less than $70 \mathrm{Myr}$ ago (Durrell et al. 2004). We therefore do not apply a correction for this object.

We provide the detailed properties of these new objects in Table 3, If we assume all candidates are group members at the distance of $\mathrm{M} 81$, we find $\mathrm{M}_{r^{\prime}}$ magnitudes range from -6.7 for d0944+69 to -13.3 for d1012+64. Half-light radii range from 90 to $490 \mathrm{pc}$ for objects well fit with Sersic functions. Because we do not yet have distances to these galaxies, we estimate that intrinsic magnitudes and radii can vary by as much as 0.14 mag and $6 \%$ in size (assuming a distance range of $\pm \mathrm{r}_{2 t}$ ). We provide $r^{\prime}$ total magnitudes of previously known M81 group members that lie within our survey region in Table 4. For the largest galaxies, we expect larger magnitude measurement errors, up to $1 \mathrm{mag}$, due to large errors in the local sky determination and subsequent negative features in the images. For measuring the local sky backgrounds, the TERAPIX reduction pipeline implements box sizes much smaller than the size of our largest galaxies.

\subsection{M81 Group Luminosity Function}

To construct the M81 group differential luminosity function (LF), we bin number counts as a function of magnitude in 2-magnitude bins. Such large bin sizes are necessary because of the small number counts we are working with and because of large magnitude measurement errors $(>0.5 \mathrm{mag})$. Counts are normalized by the size of our survey region. We construct 
luminosity functions for all previously known members, all previously known plus all new candidates from this work, and for previously known plus best candidates from this work. In the case of 'best', we include objects in Figures 445. We have excluded counts for objects we believe may be background or which could potentially prove to be artifacts. Of the previously known objects, we also exclude from this 'best' sample Arp's loop and Garland since we do not have reliable magnitude estimates for these likely tidal objects. The luminosity functions for these different samples are displayed in Figure 26. We have constructed the LFs using measured magnitudes (top), exponential profile corrected total magnitudes (middle), and Sersic profile corrected total magnitudes (bottom).

Based on our artificial galaxy tests, we shade the region where incompleteness sets in. Brighter than $r^{\prime}=17$, we expect we are $100 \%$ complete, and to $r^{\prime}=18$, to be at least $90 \%$ complete. We may still be missing a small number of dwarfs which are lurking behind bright stars or other galaxies, but find this should be less than a $4 \%$ effect, primarily affecting only the smallest objects. We use our simulations to determine estimates for our completeness at each magnitude bin in the LF. The lower limits in the detection efficiency imply that, at least in regions with good seeing and low extinction, we should recover all artificial galaxies down to $r^{\prime}=17$. At magnitudes brighter than this, we find no candidate dwarf galaxies with effective radii greater than 30 arcsec. Fainter galaxies will presumably be smaller. As a conservative estimate, we assume a maximum $\mathrm{R}_{e}$ of 35 arcsec and uniform distribution in $\mathrm{R}_{e}$ and calculate the fraction of galaxies we would expect to recover in each 2-magnitude bin of our luminosity function. In reality, we expect galaxies to be weighted towards smaller sizes at fainter magnitudes. The assumption of a uniform distribution should therefore provide us with an upper limit for the fraction of the real population we would expect to have missed, and an upper limit for corrected counts. Results are plotted in Figure 27. The solid line is a fit to the recovery results of simulations with good seeing, low extinction, and falling on full integration regions. The dashed line incorporates the $15 \%$ of the survey with single exposures and takes into account any lower completeness due to worse seeing and extinction in each field. We apply these fractions as completeness corrections to the counts in our luminosity function (open circles in Figure 26). Applied corrections also include a $3 \%$ correction for areal coverage by brighter objects for $r^{\prime} \geq 17.0$. Although these are only crude corrections since we do not know the true distribution of M81 group galaxy sizes faintward of our $100 \%$ completeness limits, we do not find any evidence suggesting the faint-end slope steepens beyond what we measure from our observed galaxy counts.

The luminosity distribution at the faint end is traditionally modeled by a power law. To measure the faint-end slope of the magnitude distribution, we perform a power law fit to the complete set of galaxy counts between $8<r^{\prime}<18$ and find a faint-end slope, $\alpha$, ranging from $-1.26_{-0.08}^{+0.09}$ for the case of measured magnitudes to a maximum of $-1.39_{-0.05}^{+0.05}$ 
for exponential profile corrected total magnitudes. The slope for the full, completeness corrected sample ranges from $-1.28_{-0.05}^{+0.04}$ to $-1.32_{-0.05}^{+0.04}$. We measure a slightly shallower faintend slope for only previously known group members of $-1.19_{-0.07}^{+0.07}$ to $-1.26_{-0.08}^{+0.08}$. However, it is clear from the figures that the slope does not increase much with the addition of these new candidate dwarf group members; only the completeness has shifted to fainter limiting magnitudes. Completeness corrected counts as a function of both measured and Sersic corrected magnitudes are well described by $\alpha=-1.3$ slopes. Best fit values for the faint-end slope, $\alpha$, for all samples are listed in Table 5 .

Due to the overall small number counts of group galaxies, uncertainties in each bin of our differential luminosity function are large. This has the effect of producing a number count distribution that does not monotonically rise as a function of magnitude and, with so few datapoints, is not well fit with a power law. We therefore also produce a plot of the unbinned cumulative distribution of galaxy counts as a function of measured and corrected total magnitude for all subsamples in Figure 28, We fit a cumulative Schechter function to the cumulative counts,

$$
\int \Phi(M) d M=0.4 \ln 10 \phi_{*} \int 10^{0.4(\alpha+1)\left(M_{*}-M\right)} e^{-10^{0.4\left(M_{*}-M\right)}} d M
$$

such that

$$
N(<M)=\phi_{*} \gamma\left[\alpha+1,10^{0.4\left(M_{*}-M\right)}\right]
$$

using maximum likelihood techniques with a Poisson estimator. $M_{*}$ and $\phi_{*}$ are not well constrained due in part to large errors in magnitude at the bright end. For M81, we know our measured total magnitude is strongly affected by improper sky subtraction. For the luminosity function we therefore assume a total magnitude for this galaxy, $M_{r^{\prime}}=-21.8$, from previously obtained $R$ band data (Tully \& Pierce 2000). For fitting the cumulative Schechter function to our data, we hold $M_{*}$ and $\phi_{*}$ constant while solving for the best fit slope, $\alpha$. Results for the different samples are presented in Table 6. To test the effect of $M_{*}$ on the fit, we refit the data allowing all parameters to vary. We find the slope changes typically by about 0.015 , and no more than 0.04 , smaller than the random errors in our fitted slope values. Results are similar when we use our original total magnitude measurement for M81. Therefore, the value of $M_{*}$, although coupled to $\alpha$, does not significantly affect the determination of the faint-end slope. In Figure 29, we display the $1 \sigma \alpha-M_{*}$ error ellipses for the luminosity functions of 'best' and 'all' candidate samples with Sersic corrected magnitudes. It can be seen that $M_{*}$ is unconstrained at the bright end, but the choice of $M_{*}$ will not strongly effect the value of $\alpha$.

Overall, we obtain very similar values for the faint-end slope as compared with the power law fit to the faint end of the differential LF. For all samples, we find a faint-end slope, 
$\alpha$, of $\sim-1.28$. Slopes for only originally known and 'best' candidates are insignificantly shallower than for all candidates. We furthermore find the faint-end slope to be robust against corrections from measured to total magnitude.

Fits to completeness corrected counts do not produce steeper faint-end slopes, but do extend our fits to all counts down to $r^{\prime} \sim-7$. Since we expect these completeness corrections are already an upper limit given the expected true size distribution of real galaxies, we do not believe the slope steepens appreciably at magnitudes fainter than our completeness limit. The steepest measured slopes are for the full sample of objects which assume all candidate objects we have detected are real galaxies within the M81 group. Certainly some subset of these candidates are not true M81 group galaxies, so we can take these slopes as an upper limit. We find the best fit slope for the subsample of most likely M81 members using Sersic profile corrected magnitudes to be $-1.30 \pm 0.10$ and $-1.26 \pm 0.05$ for the differential and integrated LFs respectively.

\section{Discussion}

\subsection{Dwarf Population Characteristics}

Of the new candidate galaxies, roughly $45 \%$ are believed to belong to the dwarf spheroidal class with dominant old stellar populations, while the rest exhibit dwarf irregular or BCD morphology with younger stellar populations and ongoing/recent star formation. At least one new object may be a tidal dwarf, formed in the tidal streams connecting the three closely interacting galaxies of M81, M82, and NGC 3077. This area contains a number of knots of star formation and tidal debris. Previously known or suspected tidal objects include Arp's loop knots, Garland knots, Ho IX, and BK3N (Makarova et al. 2002). These objects are found to be dominated by young stellar populations and have high gas contents (de Mello et al. 2008; Sabbi et al. 2008). Davidge (2008) recently found an arc of stars in the M82 halo which may have formed around the same time as these other tidal features. One of our candidate objects, d0959+68, was first noted by Durrell et al. (2004) in a search for intragroup stars in the field of the M81-NGC $3077 \mathrm{HI}$ tidal tail. It has recently undergone a burst of star formation as evidenced by the large population of bright blue stars seen in Figure 19. Durrell et al. (2004) match isochrones to the CMD of this object and find evidence that star formation occurred 30-70 Myr ago, after the formation of the HI tidal arm. In a search for HI clouds in the M81 group, Brinks et al. (2007) find a number of free floating clouds without optical counterparts which they suggest may be tidal debris from the 3-body interaction involving M81, M82, and NGC 3007. One of these clouds is just offset from d0959+68, about $1^{\prime} .5$ arcmin to the SW, several times the measured optical 
half-light radius. This HI cloudlet has a radial velocity distinct from both the Milky Way and M81 tidal arm. If it turns out the optical component has a similar radial velocity as the HI cloud, it would be likely that the two were associated. It may be possible that, like the Local Group Phoenix dwarf (Young et al. 2007), blow out from supernovae energetics from this young stellar population has displaced the neutral hydrogen gas. We display this new object along with previously suspected tidal dwarf galaxies superimposed on the HI map of Yun et al. (1994) in Figure 30.

Three new M81 candidate member galaxies (d1028+70, d0958+66, d1012+64) are of the poorly understood blue compact dwarf class. Spectra of these galaxies were obtained with Subaru/FOCAS and BTA/SCORPIO. Radial velocities of $\sim+60,-100$, and +150 $\mathrm{km} \mathrm{s}^{-1}$ support group membership for d0958+66, d1028+70 and d1012+64 respectively. All spectra exhibit strong Balmer absorption lines indicative of recent star formation. The BTA $6 \mathrm{~m}$ spectra also find $\mathrm{H} \alpha$ emission in all three cases indicating active star formation, in agreement with the blue $(V-I) \sim 0.8$ colors found for these galaxies. These three candidates bring the total number of M81 group BCDs to at least four, including UGC 6456, long considered an isolated member of the M81 group (Lynds et al. 1998; Tully et al. 1981). DDO 82 may have a BCD component as well. It has an optical structure and color that corresponds to that of BCDs but has an HI flux contaminated by local HI. UGC 6456 lies far outside our survey region, at $\mathrm{SGL}=37^{\circ}, \mathrm{SGB}=+11^{\circ}$ in Figure 1, while DDO 82 lies at the projected distance of the second turnaround radius for the M81 group. BCDs tend to be found more frequently in field environments than clusters and are one of the least clustered galaxy types known (van Zee et al. 2001). The three BCDs discovered here all lie in low density regions at a minimum projected distance of $140 \mathrm{kpc}$ from M81, with d1012+64 lying outside of the second turnaround radius at a projected distance of $315 \mathrm{kpc}$. The excess of this type of galaxy observed in this group may indicate that the termination of star formation is due to processes such as ram pressure stripping which occur preferentially in more massive and more dynamically evolved groups/clusters. However, given the projected distances of the M81 BCD population, it is possible that these galaxies are on first infall orbits and processes such as strangulation that serve to shut down star formation even in poor groups (Kawata \& Mulchaev 2008) have yet to act on these galaxies.

We find no ultra-compact dwarfs (UCDs) such as those found in the richer Fornax and Virgo clusters (Drinkwater et al. 2000, 2003). Neither have UCDs been discovered in the Local Group. This may support the hypothesis that UCD formation is driven by a mechanism that occurs preferentially in denser environments, such as tidal stripping of nucleated dwarf ellipticals (Bekki et al. 2001a) or late-type spirals (Moore et al. 1998). Alternatively, Fellhauer \& Kroupa (2002) suggest the possible formation of UCDs as objects evolved from young massive star clusters which are formed within tidals tails of massive galaxy-galaxy 
mergers. In this scenario, one might expect the formation of such objects to be ongoing in the tidal tails of the galaxy interactions occuring in the M81 group although we find no evidence for this. It is possible that we would be unable to detect the more compact UCDs in this survey using our current search techniques. We show in Figure 12 the location in $r^{\prime}-\mu_{e}$ space the UCDs would occupy. Our detection limit would enclose only the brighter and larger UCDs. Due to the extremely compact nature of UCDs, it is probable that the smaller ones would not appear in our images as resolved concentrations of stars. Rather, the light on these small scales would be blended making detection based on a resolved stellar population difficult and in some cases impossible. However, we would expect to be able to detect the larger and brighter subpopulation. As we do not detect a single UCD, this suggests that UCDs are absent in this poor, unevolved environment.

No new bright $\left(r^{\prime}<-12\right)$ ultra-diffuse dwarfs like F8D1 and IKN have been found in this survey. This could partially be due to selection effects since neither our eye nor the twopoint correlation code would be particularly sensitive to extremely diffuse objects, although these two objects were easily picked out both by eye and recovered with the two-point correlation technique. We would therefore expect to detect similarly low density objects if they existed. We do find a number of very diffuse, faint, and small candidates including $\mathrm{d} 1013+68, \mathrm{~d} 1014+68$, and $\mathrm{d} 1016+69$ which may or may not prove to be real objects.

In comparison with the Local Group, we find a very different morphological mix of dwarf satellites. In the M81 group, we have found 3 new BCD candidates while no such objects have been discovered around M31 or the Milky Way. The fraction of late type, dwarf irregulars is also much higher in the M81 group. Including the recent detections of ultrafaint dwarfs in the LG, the fraction of late types is $\sim 25 \%$ while in the M81 group, including our new best candidates, the fraction of late types could be as high as $55 \%$. This different morphological mix had been noted earlier for giant galaxies and brighter dwarfs, but recently discovered faint dwarfs in both groups maintain the distinctly different population fractions. It is inferred that the M81 group is dynamically less evolved than the Local Group.

We do not find any objects as faint as the recently discovered ultra-faint Local Group galaxies. This is due to incompleteness at these low magnitudes. We do find one candidate, $\mathrm{d} 0944+69$, with an absolute $\mathrm{r}^{\prime}$ magnitude of $-6.7 \pm 0.5$. This would make it the faintest known M81 group dwarf. It also would have one of the smallest sizes for known dwarfs of $\mathrm{R}_{e}=90 \mathrm{pc}$, similar in size to some of the recent Local Group satellite discoveries with $\mathrm{R}_{e}$ ranging from $23-125$ pc (Willman et al. 2005; Zucker et al. 2006; Martin et al. 2006; Belokurov et al. 2007a; Walsh et al. 2007). We do not yet know if this object is either a real galaxy or a member of the M81 group. If this object does turn out to be a group member, its lower limit half-light radius would impinge on the $40<R_{e}<100$ pc 'size gap' between 
dwarfs and globular clusters (Belokurov et al. 2007a). However, as most of the galaxy light likely falls below the level of the sky, we are certainly underestimating its physical size and perhaps, even with the magnitude correction, total luminosity.

The other candidates all have properties consistent with normal faint dwarfs. We plot $M_{r^{\prime}}$ vs $\log$ (half-light radius) in Figure 31. New candidates are shown as filled circles (assuming a distance modulus of 27.8) while previously known group members are displayed as filled squares. The three candidate BCDs are distinguished by their high surface brightnesses. For comparison, we include Local Group dwarfs in this plot. Recent discoveries are denoted by open circles while previously known galaxies are shown as open squares. To transform from $V$ to $r^{\prime}$ magnitudes, we use $r^{\prime}=V-0.84(V-R)+0.13$ (Fukugita et al. 1996b) assuming an approximate $(V-R)$ color of 0.6 for dwarf galaxies. Lines of constant surface brightness are shown. New Local Group detections only became possible with the SDSS survey reaching effective surface brightnesses below $27.0 \mathrm{mag}_{V} \operatorname{arcsec}^{-2}$ (Belokurov et al. 2007b), corresponding to a stellar surface density limit. Incompleteness in our survey may be starting to set in by 26.0 mag $_{r^{\prime}} \operatorname{arcsec}^{-2}$ and most of our detections are brighter than $27.0 \mathrm{mag}_{r^{\prime}}$ $\operatorname{arcsec}^{-2}$, so we may still be missing a large population of ultra-faint dwarfs. According to Figures 12 and 31, we would expect these missing galaxies to have total magnitudes fainter than $M_{r^{\prime}} \sim-8$.

\subsection{Distribution of M81 galaxies}

The asymmetric distribution of dSphs noted by Karachentsev et al. (2000) has become less pronouced with the addition of the new objects. As discussed in that paper, a possible cause for the asymetry is contamination of the M81 group area with many reflecting nebulae (galactic cirrus) which made it difficult to find extremely LSB dwarfs unresolved into stars. In Figure 32 we show the M81 region of the sky in the Schlegel et al. (1998) galactic dust map. Superimposed are the locations of galaxies in the group. A number of new candidates (red crosses) can be seen to lie in regions of higher extinction and, given the dearth of detections in regions with the highest extinction, it is possible we are still missing faint M81 dwarfs concealed by these clouds. However, it seems unlikely we would be missing dwarfs of comparable brightness to the ones displaying the noted asymetry, given that the new candidates from this survey which are detected in regions of high extinction are all 1-2 mags fainter.

We compare the projected distribution of morphological types in Figure 33. Late types, early types, and tidal candidates are distinguished by color while point sizes indicate surface brightness with brighter surface brightnesses denoted by larger points. All the bright giant 
galaxies are late types (blue) and all candidate tidal dwarfs (green) lie near the center of the cluster right around M81, M82, and NGC 3077. Early type dwarf spheroidals (red) lie primarily towards the group core while all late type dwarfs lie towards the periphery of the group. A clear morphology-density relation is seen. We find the median distance from M81 for late type dwarfs is 1.7 times that for dwarf spheroidals.

\subsection{Implication for Cosmological Models}

$\Lambda$ CDM models predict a faint-end slope for the mass function of -1.8 (Trentham \& Tully 2002). We measure a luminosity function faint-end slope, $\alpha \sim-1.28 \pm 0.06$ for M81 group galaxies. The steepest faint-end slope consistent with our data is found to be $-1.39 \pm 0.05$ for our binned differential LF, constructed with all candidates and having presumably overestimated magnitude corrections under the assumption of exponential profiles. Even when we apply completeness corrections at faint magnitudes, we find faint-end slopes flatter than -1.30. When we include only most likely member candidates in our LF, we find a slightly shallower slope of $-1.26 \pm 0.05$. We therefore expect -1.30 to be an upper limit.

Assuming the LF follows a constant slope of -1.3 , rather than turning over at $r^{\prime}>17.0$ where we start to become incomplete, we would expect to be missing at least $\sim 70$ M81 group members in our survey area in the range $17<r^{\prime}<22\left(-11<M_{r^{\prime}}<-6\right)$. If the slope increased to -1.8 at faint magnitudes, beginning at $M_{r}^{\prime}=-12$, we would expect to be missing over 1700 galaxies in our survey area. This is over an order of magnitude greater than we would expect from our data. For the intrinsic faint-end slope to be this steep, we would have to be missing a very large population of very faint and low surface brightness, low stellar concentration galaxies, and based on our simulations, we do not find any evidence that we could be missing such large numbers of galaxies brighter than $M_{r^{\prime}}=-7$.

We compare the M81 group LF with that of other nearby groups. Detections of dwarf galaxies in the LG go much fainter than we recover in M81. However, because of the full sky coverage necessary to obtain a complete census, completeness is still quite low. We therefore look only at the LF for Andromeda and its satellites. Large areal coverage is still required, including probing into the zone of obscuration, but progress is being made. We construct a cumulative LF for Andromeda satellites including all previously known galaxies (van den Bergh 2006) and recent family additions (Ibata et al. 2007; Majewski et al. 2007; Martin et al. 2006; Irwin et al. 2008) (Figure 34). A fit to the cumulative $V$ band counts finds a faint-end slope of $-1.12_{-0.06}^{+0.06}$. This is very similar to the best Schechter function fit for LG galaxies found by Pritchet \& van den Bergh (1999) with $\alpha=-1.1 \pm 0.1$. 
The nearby Cen A group at 3.6 Mpc, in contrast to the spiral dominant LG and M81 groups, contains a dominant giant elliptical galaxy with radio loud AGN and a late type fraction of only $\sim 38 \%$. Using $B$ band magnitudes for the Cen A group from Karachentsev et al. $(2002 \mathrm{~b})$, we find a faint-end slope $\alpha=-1.22_{-0.10}^{+0.04}$, nearly as steep as that of M81 (Figure 34).

Boyce et al. (2001) find that steep LFs are generated by the combined number counts of $\mathrm{dI}$ and $\mathrm{dE}$ galaxies, and when dIs are excluded from the construction of the LF, the faint-end slope flattens. This might imply that more evolved regions with fewer late types should have shallower slopes. Alternatively, from a comparison of poor groups, clusters, and field environments, Zabludoff \& Mulchaev (2000) found that the dwarf-to-giant ratio (and therefore the faint end of the LF) increased in regions of higher density, and that this increase was specifically due to an increase in the fraction of quiescent dwarfs. In our work on M81 we find a steeper slope in the dynamically unevolved, dI-rich M81 group than these other two groups and in particular the dSph-rich LG. However, recent work on the luminosity function for two nearby dynamically evolved groups with large early type galaxy populations finds steep faint-end slopes with values of $-1.3 \pm 0.1$ for the NGC 5846 group (Mahdavi et al. 2005) and -1.35 for the NGC 1407 group (Trentham et al. 2006), slightly steeper than what we find for M81. These works determine group membership on the basis of morphology and surface brightness and both reach down to $M_{R}=-12$. It is possible that the shallower LG $\mathrm{LF}$ is due in part to incompleteness in the surveyed region. In recent work, Koposov et al. (2007) construct the LF for the Local Group including the recent discoveries of ultra-faint dwarfs with SDSS. They also include a volume correction factor, assuming different number density laws for the distribution of halo satellites, and find faint-end slopes ranging between $-1.29<\alpha<-1.25$ within the magitude range $-19<M_{V}<-3$, very similar to the slope we find for M81. In this case, with all groups discussed here having faint end slopes of $\alpha \sim-1.3$, we find no clear trend in faint end slope as a function of environment within groups of galaxies.

Using a statistical subtraction of the background for nearby groups and poor clusters identified in SDSS data, González et al. (2006) find a sharp upturn in composite LFs at $M_{r} \sim-17$ with slopes of $-1.9<\alpha<-1.6$. They find this upturn regardless of group mass, number of members, or environment. If the slope were this steep in our survey region, we would expect to detect the large numbers of bright dwarf galaxies implied by this slope. Down to at least $M_{r^{\prime}}<-10$ we find no such upturn in the poor M81 group when constructing the LF from our nearly complete survey of galaxies whose membership is established through more direct means.

Koposov et al. (2007) compare the Local Group LF to semi-analytical predictions and 
find a reasonable match with Benson et al. (2003) who include effects of tidal disruption and photoionization to CDM theory predictions. Counts to $M_{V}<-3$ agree well within the uncertainties. This provides some support for the hypothesis that at least part of the cause of the disparity between $\Lambda \mathrm{CDM}$ predictions and observations is due to the suppression of gas accumulation in small galaxies collapsing post reionization although other predictions from their model, such as central surface brightness values, do not agree well with observations. In further support of this idea, Simon \& Geha (2007) compare the observed LG circular velocity distribution function (a proxy for the mass function) to that predicted from the Via Lactea N-body simulation. They find good agreement when assuming that the LG halos observed at $\mathrm{z}=0$ were the ones which collapsed prior to reionization and which correspond to the model objects with the largest values of $v_{\text {circ }}$ at various high redshifts. This result constrains the reionization era to $\mathrm{z}>8.0$, in agreement with WMAP results.

We find a faint-end slope of the M81 group LF to a limiting magnitude of $M_{r^{\prime}}=-10$ that is much shallower than CDM cosmology would predict. Part of the problem may be in the physics of how mass is converted to light. Incorporating the effects of feedback and star formation efficiency may help reconcile this difference. Accounting for the suppression of gas infall into the low mass halos of the forming galaxies by reionization in the early universe may explain the bulk of the discrepancy and naturally explains the noted environmental dependence of the LF, with clusters and more dynamically evolved regions exhibiting slopes with $\alpha$ steeper than -1.4 (see e.g. Trentham et al. (2005); Conselice (2002); Chiboucas \& Mateo (2006); de Propris \& Pritchet (1998); Bernstein et al. (1995)), in contrast to what we find for the poorer groups.

\section{Summary and Conclusions}

We have discovered 22 new candidate dwarf galaxies in the M81 group from our 65 square degree CFHT/MegaCam survey, designed to extend beyond the second turnaround radius for the group. Of these candidates, we believe 12 are likely members. The remainder may prove to be background galaxies, foreground associations of stars, or artifacts in our data. The 12 likely members consist of 3 candidate BCDs lying near the periphery of the group, 1 tidal dwarf candidate lying within the HI tidal bridge between M81 and NGC 3077, and a number of $\mathrm{dI}$ and $\mathrm{dSph}$ candidates. No new large diffuse or any ultra compact dwarfs are detected. The large fraction of late to early types previously noted in the M81 group is reconfirmed with our new objects. Assuming all objects lie at the distance of M81, we find $r^{\prime}$ absolute magnitudes for these new objects range from -6.7 to -13.3. The faintest object, if real, has a measured size of $90 \mathrm{pc}$ that would cause it to encroach on the $40-100$ pc size 
gap region between globular clusters and dwarf galaxies. From false star/galaxy tests we expect to detect nearly $100 \%$ of the group member dwarfs down to $M_{r^{\prime}}=-10$ and over $50 \%$ to $M_{r^{\prime}}=-9$, not quite into the regime of the recently discovered ultra faint LG galaxies. Including all 22 previously known M81 group members in our survey region, we construct the group differential and cumulative luminosity functions and find modestly steep faint-end slopes of $-1.30 \pm 0.06$. Including only the most likely members, we find a similar slope of $-1.27 \pm 0.06$. This is steeper than what has been found for the Andromeda satellites, but may be consistent with the Local group after taking into account all new discoveries and including corrections for completeness. Our dwarf number counts are still an order of magnitude below cosmological predictions, but may agree with theory when reionization effects on collapsing primordial galaxies are taken into account.

This research was funded in part by NSF award AST03-07706 and RFBR grant 0702-00005. Thanks to Canada-France-Hawaii Telescope queue observers, TERAPIX at the Institut d'Astrophysique de Paris, and Subaru Telescope support staff, Hélène Courtois and Luca Rizzi for UH88 observations, Viktor Afanasiev and Alex Moiseev for work with the BTA $6 \mathrm{~m}$ data, and Elias Brinks for providing information on an HI cloudlet near one of our candidates.

\section{REFERENCES}

Afanasiev, V. L. \& Moiseev, A. V. 2005, Astronomy Letters, 31, 194

Allen, R. J. \& Shu, F. H. 1979, ApJ, 227, 67

Bekki, K., Couch, W. J., \& Drinkwater, M. J. 2001a, ApJ, 552, L105

Bekki, K., Couch, W. J., \& Shioya, Y. 2001b, PASJ, 53, 395

Belokurov, V., Zucker, D. B., Evans, N. W., Kleyna, J. T., Koposov, S., Hodgkin, S. T., Irwin, M. J., Gilmore, G., Wilkinson, M. I., Fellhauer, M., Bramich, D. M., Hewett, P. C., Vidrih, S., De Jong, J. T. A., Smith, J. A., Rix, H.-W., Bell, E. F., Wyse, R. F. G., Newberg, H. J., Mayeur, P. A., Yanny, B., Rockosi, C. M., Gnedin, O. Y., Schneider, D. P., Beers, T. C., Barentine, J. C., Brewington, H., Brinkmann, J., Harvanek, M., Kleinman, S. J., Krzesinski, J., Long, D., Nitta, A., \& Snedden, S. A. 2007a, ApJ, 654, 897

-. 2007b, ApJ, 654, 897 
Benson, A. J., Frenk, C. S., Baugh, C. M., Cole, S., \& Lacey, C. G. 2003, MNRAS, 343, 679

Bernstein, G. M., Nichol, R. C., Tyson, J. A., Ulmer, M. P., \& Wittman, D. 1995, AJ, 110, $1507+$

Boerngen, F. \& Karachentseva, V. E. 1982, Astronomische Nachrichten, 303, 189

—. 1985, Astronomische Nachrichten, 306, 301

Boyce, P. J., Phillipps, S., Jones, J. B., Driver, S. P., Smith, R. M., \& Couch, W. J. 2001, MNRAS, 328, 277

Brinks, E., Walter, F., \& Skillman, E. D. 2007, ArXiv e-prints, 708

Caldwell, N., Armandroff, T. E., Da Costa, G. S., \& Seitzer, P. 1998, AJ, 115, 535

Caon, N., Capaccioli, M., \& D’Onofrio, M. 1993, MNRAS, 265, 1013

Chiboucas, K. \& Mateo, M. 2006, AJ, 132, 347

—. 2007, ApJS, 170, 95

Colín, P., Avila-Reese, V., \& Valenzuela, O. 2000, ApJ, 542, 622

Conselice, C. J. 2002, ApJ, 573, L5

Davidge, T. J. 2008, ArXiv e-prints, 803

de Mello, D. F., Smith, L. J., Sabbi, E., Gallagher, J. S., Mountain, M., \& Harbeck, D. R. 2008, AJ, 135, 548

de Propris, R. \& Pritchet, C. J. 1998, AJ, 116, 1118

Drinkwater, M. J., Gregg, M. D., Hilker, M., Couch, W. J., Ferguson, H. C., Jones, B., \& Phillipps, S. 2003, The Cosmic Cauldron, 25th meeting of the IAU, Joint Discussion 10, 18 July 2003, Sydney, Australia, 10

Drinkwater, M. J., Jones, J. B., Gregg, M. D., \& Phillipps, S. 2000, Publications of the Astronomical Society of Australia, 17, 227

Durrell, P. R., Decesar, M. E., Ciardullo, R., Hurley-Keller, D., \& Feldmeier, J. J. 2004, in IAU Symposium, Vol. 217, Recycling Intergalactic and Interstellar Matter, ed. P.-A. Duc, J. Braine, \& E. Brinks, 90-+

Fellhauer, M. \& Kroupa, P. 2002, MNRAS, 330, 642 
Froebrich, D. \& Meusinger, H. 2000, A\&AS, 145, 229

Fukugita, M., Ichikawa, T., Gunn, J. E., Doi, M., Shimasaku, K., \& Schneider, D. P. 1996a, AJ, 111, 1748

-. 1996b, AJ, 111, 1748

González, R. E., Lares, M., Lambas, D. G., \& Valotto, C. 2006, A\&A, 445, 51

Haiman, Z., Thoul, A. A., \& Loeb, A. 1996, ApJ, 464, 523

Ibata, R., Martin, N. F., Irwin, M., Chapman, S., Ferguson, A. M. N., Lewis, G. F., \& McConnachie, A. W. 2007, ApJ, 671, 1591

Irwin, M., Ferguson, A., Huxor, A., Tanvir, N., Ibata, R., \& Lewis, G. 2008, ArXiv e-prints, 802

Irwin, M. \& Hatzidimitriou, D. 1995, MNRAS, 277, 1354

Jerjen, H. 1995, Ph.D. Thesis

Jordi, K., Grebel, E. K., \& Ammon, K. 2006, A\&A, 460, 339

Kamionkowski, M. \& Liddle, A. R. 2000, Physical Review Letters, 84, 4525

Karachentsev, I. D., Dolphin, A. E., Geisler, D., Grebel, E. K., Guhathakurta, P., Hodge, P. W., Karachentseva, V. E., Sarajedini, A., Seitzer, P., \& Sharina, M. E. 2002a, A\&A, 383, 125

Karachentsev, I. D., Karachentseva, V. E., Dolphin, A. E., Geisler, D., Grebel, E. K., Guhathakurta, P., Hodge, P. W., Sarajedini, A., Seitzer, P., \& Sharina, M. E. 2000, A\&A, 363, 117

Karachentsev, I. D., Karachentseva, V. E., Huchtmeier, W. K., \& Makarov, D. I. 2004, AJ, 127,2031

Karachentsev, I. D., Sharina, M. E., Dolphin, A. E., Grebel, E. K., Geisler, D., Guhathakurta, P., Hodge, P. W., Karachentseva, V. E., Sarajedini, A., \& Seitzer, P. 2002b, A\&A, 385, 21

Karachentseva, V. E. 1968, Soobshcheniya Byurakanskoj Observatorii Akademiya Nauk Armyanskoj SSR Erevan, 39, 62

Kawata, D. \& Mulchaey, J. S. 2008, ApJ, 672, L103 
Kewley, L. J. \& Dopita, M. A. 2002, ApJS, 142, 35

Klypin, A., Kravtsov, A. V., Valenzuela, O., \& Prada, F. 1999, ApJ, 522, 82

Koposov, S., Belokurov, V., Evans, N. W., Hewett, P. C., Irwin, M. J., Gilmore, G., Zucker, D. B., Rix, H. ., Fellhauer, M., Bell, E. F., \& Glushkova, E. V. 2007, ArXiv e-prints, 706

Kurtz, M. J. \& Mink, D. J. 1998, PASP, 110, 934

Lynds, R., Tolstoy, E., O’Neil, Jr., E. J., \& Hunter, D. A. 1998, AJ, 116, 146

Mahdavi, A., Trentham, N., \& Tully, R. B. 2005, AJ, 130, 1502

Majewski, S. R., Beaton, R. L., Patterson, R. J., Kalirai, J. S., Geha, M. C., Muñoz, R. R., Seigar, M. S., Guhathakurta, P., Gilbert, K. M., Rich, R. M., Bullock, J. S., \& Reitzel, D. B. 2007, ApJ, 670, L9

Makarova, L. N., Grebel, E. K., Karachentsev, I. D., Dolphin, A. E., Karachentseva, V. E., Sharina, M. E., Geisler, D., Guhathakurta, P., Hodge, P. W., Sarajedini, A., \& Seitzer, P. 2002, A\&A, 396, 473

Martin, N. F., Ibata, R. A., Irwin, M. J., Chapman, S., Lewis, G. F., Ferguson, A. M. N., Tanvir, N., \& McConnachie, A. W. 2006, MNRAS, 371, 1983

Mateo, M. L. 1998, ARA\&A, 36, 435

McConnachie, A. W. \& Irwin, M. J. 2006, MNRAS, 365, 1263

Monet, D. G., Levine, S. E., Canzian, B., Ables, H. D., Bird, A. R., Dahn, C. C., Guetter, H. H., Harris, H. C., Henden, A. A., Leggett, S. K., Levison, H. F., Luginbuhl, C. B., Martini, J., Monet, A. K. B., Munn, J. A., Pier, J. R., Rhodes, A. R., Riepe, B., Sell, S., Stone, R. C., Vrba, F. J., Walker, R. L., Westerhout, G., Brucato, R. J., Reid, I. N., Schoening, W., Hartley, M., Read, M. A., \& Tritton, S. B. 2003, AJ, 125, 984

Moore, B., Ghigna, S., Governato, F., Lake, G., Quinn, T., Stadel, J., \& Tozzi, P. 1999, ApJ, 524, L19

Moore, B., Katz, N., Lake, G., Dressler, A., \& Oemler, A. 1996, Nature, 379, 613

Moore, B., Lake, G., \& Katz, N. 1998, ApJ, 495, 139

Mori, M. \& Burkert, A. 2000, ApJ, 538, 559 
Perelmuter, J.-M. \& Racine, R. 1995, AJ, 109, 1055

Pettini, M. \& Pagel, B. E. J. 2004, MNRAS, 348, L59

Press, W. H., Teukolsky, S. A., Vetterling, W. T., \& Flannery, B. P. 1992, Numerical recipes in FORTRAN. The art of scientific computing (Cambridge: University Press, —c1992, 2nd ed.)

Pritchet, C. J. \& van den Bergh, S. 1999, AJ, 118, 883

Sabbi, E., Gallagher, J. S., Smith, L. J., de Mello, D. F., \& Mountain, M. 2008, ArXiv e-prints, 802

Schlegel, D. J., Finkbeiner, D. P., \& Davis, M. 1998, ApJ, 500, 525+

Secker, J. \& Harris, W. E. 1997, PASP, 109, 1364

Secker, J., Harris, W. E., \& Plummer, J. D. 1997, PASP, 109, 1377

Shaya, E. J. \& Tully, R. B. 1984, ApJ, 281, 56

Simon, J. D. \& Geha, M. 2007, ArXiv e-prints, 706

Stoehr, F., White, S. D. M., Tormen, G., \& Springel, V. 2002, MNRAS, 335, L84

Strigari, L. E., Bullock, J. S., Kaplinghat, M., Diemand, J., Kuhlen, M., \& Madau, P. 2007, ApJ, 669, 676

Thoul, A. A. \& Weinberg, D. H. 1996, ApJ, 465, 608

Trentham, N. 1997, MNRAS, 286, 133

Trentham, N., Sampson, L., \& Banerji, M. 2005, MNRAS, 357, 783

Trentham, N. \& Tully, R. B. 2002, MNRAS, 335, 712

Trentham, N., Tully, R. B., \& Mahdavi, A. 2006, MNRAS, 369, 1375

Tully, R. B., Boesgaard, A. M., Dyck, H. M., \& Schempp, W. V. 1981, ApJ, 246, 38

Tully, R. B. \& Pierce, M. J. 2000, ApJ, 533, 744

Tully, R. B., Somerville, R. S., Trentham, N., \& Verheijen, M. A. W. 2002, ApJ, 569, 573

Tully, R. B., Verheijen, M. A. W., Pierce, M. J., Huang, J.-S., \& Wainscoat, R. J. 1996, AJ, 112, 2471 
van den Bergh, S. 1966, AJ, 71, 922

—. 2006, AJ, 132, 1571

van Zee, L., Salzer, J. J., \& Skillman, E. D. 2001, AJ, 122, 121

Walsh, S. M., Jerjen, H., \& Willman, B. 2007, ApJ, 662, L83

Willman, B., Blanton, M. R., West, A. A., Dalcanton, J. J., Hogg, D. W., Schneider, D. P., Wherry, N., Yanny, B., \& Brinkmann, J. 2005, AJ, 129, 2692

Young, L. M., Skillman, E. D., Weisz, D. R., \& Dolphin, A. E. 2007, ApJ, 659, 331

Yun, M. S., Ho, P. T. P., \& Lo, K. Y. 1994, Nature, 372, 530

Zabludoff, A. I. \& Mulchaey, J. S. 2000, ApJ, 539, 136

Zucker, D. B., Belokurov, V., Evans, N. W., Kleyna, J. T., Irwin, M. J., Wilkinson, M. I., Fellhauer, M., Bramich, D. M., Gilmore, G., Newberg, H. J., Yanny, B., Smith, J. A., Hewett, P. C., Bell, E. F., Rix, H.-W., Gnedin, O. Y., Vidrih, S., Wyse, R. F. G., Willman, B., Grebel, E. K., Schneider, D. P., Beers, T. C., Kniazev, A. Y., Barentine, J. C., Brewington, H., Brinkmann, J., Harvanek, M., Kleinman, S. J., Krzesinski, J., Long, D., Nitta, A., \& Snedden, S. A. 2006, ApJ, 650, L41 
Table 1. New M81 Dwarf Candidates

\begin{tabular}{|c|c|c|c|c|c|c|}
\hline Name & $\alpha$ & $\delta \mathrm{J} 2000.0$ & $B_{t}^{\mathrm{a}}$ & $a^{\prime} \times b^{\prime b}$ & Type & Comments \\
\hline d0926+70 & 092627.9 & +703024 & 19.0 & $0.8 \times 0.6$ & $\mathrm{dIr}$ & \\
\hline $\mathrm{d} 0934+70$ & 093403.7 & +701257 & 19.3 & $0.7 \times 0.6$ & $\mathrm{dSph}$ & behind cirrus \\
\hline $\mathrm{d} 0939+71$ & 093915.9 & +711842 & 20.0 & $0.7 \times 0.6$ & dSph? & 9.4 arcmin from HoI \\
\hline d0944+69 & 094422.5 & +691240 & 21.0 & $0.2 \times 0.2$ & dSph & \\
\hline d0944+71 & 094434.4 & +712857 & 17.5 & $1.3 \times 0.8$ & $\mathrm{dIr}$ & behind brt foreground star \\
\hline d0946+68 & 094613.0 & +684255 & 20.5 & $0.2 \times 0.2$ & $\mathrm{dIr}$ & background? \\
\hline $\mathrm{d} 0955+70$ & 095513.6 & +702429 & 20.5 & $0.8 \times 0.7$ & dSph & \\
\hline $\mathrm{d} 0957+70$ & 095712.4 & +701235 & 21.0 & $0.3 \times 0.3$ & dSph? & \\
\hline d0958+66 & 095848.5 & +665059 & 16.0 & $1.6 \times 0.8$ & $\mathrm{BCD}$ & KUG $0954+670$ \\
\hline d0959+68 & 095933.1 & +683925 & 18.0 & $0.8 \times 0.6$ & $\mathrm{dIr} / \mathrm{tdl}$ & tidal? Durrell et al. (2004) \\
\hline $\mathrm{d} 1006+67$ & 100646.2 & +671204 & 21.0 & $0.7 \times 0.7$ & $\mathrm{dSph}$ & \\
\hline $\mathrm{d} 1009+70$ & 100934.9 & +703255 & 18.5 & $0.6 \times 0.5$ & dIr & semi-resolved, background? \\
\hline $\mathrm{d} 1012+64$ & 101248.4 & +640627 & 15.6 & $1.4 \times 0.9$ & $\mathrm{BCD}$ & UGC5497 \\
\hline $\mathrm{d} 1013+68$ & 101311.7 & +684345 & 20.5 & $1.5 \times 0.9$ & dIr? & artifact? \\
\hline $\mathrm{d} 1014+68$ & 101455.8 & +684527 & 21.0 & $0.4 \times 0.4$ & $\mathrm{dSph}$ & \\
\hline $\mathrm{d} 1015+69$ & 101506.9 & +690215 & 20.5 & $0.4 \times 0.3$ & dIr & backgrnd? \\
\hline $\mathrm{d} 1016+69$ & 101618.3 & +692945 & 21.0 & $0.4 \times 0.4$ & $\mathrm{dSph}$ & \\
\hline $\mathrm{d} 1019+69$ & 101952.9 & +691119 & 18.5 & $0.6 \times 0.5$ & dIr & backgrnd? \\
\hline $\mathrm{d} 1020+69$ & 102025.0 & +691150 & 20.5 & $1.5 \times 0.7$ & dSph? & artifact? \\
\hline $\mathrm{d} 1028+70$ & 102839.7 & +701401 & 16.2 & $1.4 \times 0.9$ & $\mathrm{BCD}$ & \\
\hline $\mathrm{d} 1041+70$ & 104116.8 & +700903 & 20.5 & $0.8 \times 0.4$ & $\mathrm{dIr}$ & pear-shaped \\
\hline $\mathrm{d} 1048+70$ & 104857.0 & +702538 & 21.0 & $1.0 \times 0.9$ & dSph & background? \\
\hline
\end{tabular}

${ }^{\mathrm{a}} B_{t}$ are estimated by eye on POSS-II

$\mathrm{b}$ angular dimensions correspond to an isophote $28 \mathrm{~m} \mathrm{arcsec}-2$ 
Table 2. Spectroscopy of 5 dwarfs in M81

\begin{tabular}{|c|c|c|c|c|c|c|c|c|c|}
\hline Name & $\alpha$ & $\begin{array}{c}\delta \\
(\mathrm{J} 2000.0)\end{array}$ & telescope & date & $\begin{array}{c}\text { exposure } \\
\text { (s) }\end{array}$ & grating & objects & $\begin{array}{c}\mathrm{v}_{\operatorname{rad}(\operatorname{Sub})} \\
(\mathrm{km} / \mathrm{s})\end{array}$ & $\begin{array}{c}\mathrm{v}_{\operatorname{rad}(B T A)} \\
(\mathrm{km} / \mathrm{s})\end{array}$ \\
\hline \multicolumn{10}{|c|}{ Previously known: } \\
\hline F8D1 & 94439.3 & +672606 & Subaru & 24 Nov 06 & 1800 & VPH & GC & $-125 \pm 130$ & \\
\hline IKN & 100805.2 & +682433 & Subaru & 24 Nov 06 & 1800 & VPH & GC & $-140 \pm 64$ & \\
\hline \multicolumn{10}{|c|}{ New Candidates: } \\
\hline \multirow[t]{2}{*}{ d0958+66 } & 095848.5 & +665059 & Subaru & 22 Nov 06 & 600 & R300 & galaxy & $+33 \pm 94$ & \\
\hline & & & BTA & 11 Nov 06 & 600 & VPHG400 & galaxy & & $+90 \pm 50$ \\
\hline \multirow[t]{2}{*}{$\mathrm{d} 1028+70$} & 102839.7 & +701401 & Subaru & 23 Nov 06 & 600 & VPH & galaxy & $-90 \pm 79$ & \\
\hline & & & BTA & 11 Nov 06 & 600 & VPHG400 & galaxy & & $-114 \pm 50$ \\
\hline $\mathrm{d} 1012+64$ & 101248.4 & +640627 & BTA & 20 Oct 07 & 900 & VPHG400 & galaxy & & $+150 \pm 50$ \\
\hline
\end{tabular}


Table 3. Structural and Photometric Properties of M81 Group Candidates

\begin{tabular}{|c|c|c|c|c|c|c|c|c|c|c|c|c|c|c|c|}
\hline galaxy & $r_{a p}^{\prime a}$ & $\begin{array}{l}r_{a p_{2}}^{\prime \quad \mathrm{b}} \\
\mathrm{mag}\end{array}$ & $r_{f i t}^{\prime c}$ & $(V-I)^{\mathrm{d}}$ & $\epsilon$ & PA & $\begin{array}{c}\mu_{\circ} \\
r^{\prime} \operatorname{arcsec}^{-2}\end{array}$ & $\begin{array}{c}\left\langle\mu_{e}\right\rangle \\
r^{\prime} \operatorname{arcsec}^{-2}\end{array}$ & $\begin{array}{c}\mathrm{R}_{e} \\
\operatorname{arcsec}\end{array}$ & $\begin{array}{l}\mathrm{R}_{e} \\
\mathrm{kpc}\end{array}$ & $\mathrm{n}$ & $\begin{array}{l}\mathrm{A}_{r^{\prime}} \\
\mathrm{mag}\end{array}$ & $r_{\text {cor }_{e}}^{\prime}{ }^{\mathrm{e}}$ & $\begin{array}{l}r_{\text {cors }_{s}}^{\prime} \\
\mathrm{mag}\end{array}$ & $\mathrm{M}_{r_{\text {cors }}^{\prime}} \mathrm{g}$ \\
\hline $\mathrm{d} 0926+70^{\dagger}$ & 18.3 & 18.9 & 18.4 & 0.87 & 0.18 & 67.0 & 26.0 & 26.2 & 14.3 & 0.25 & 0.42 & 0.48 & 17.4 & 17.9 & -9.9 \\
\hline $\mathrm{d} 0934+70^{\dagger}$ & 18.9 & 18.9 & 18.5 & 1.07 & 0.07 & -25.6 & 26.2 & 26.4 & 15.3 & 0.27 & 0.42 & 0.66 & 17.5 & 18.0 & -9.8 \\
\hline $\mathrm{d} 0939+71$ & 18.9 & 19.1 & 18.2 & & 0.05 & 10.0 & 25.5 & 26.1 & 15.3 & 0.27 & 0.66 & 0.09 & 18.3 & 18.5 & -9.3 \\
\hline d0944+69 & 21.6 & 21.9 & 21.2 & & 0.09 & 81.2 & 25.9 & 26.7 & 4.9 & 0.09 & 0.78 & 0.22 & 20.7 & 21.1 & -6.7 \\
\hline d0944+71 & 15.6 & 15.9 & 15.6 & & 0.20 & 63.5 & 23.4 & 23.9 & 18.3 & 0.32 & 0.61 & 0.09 & 15.0 & 15.2 & -12.6 \\
\hline d0946+68 & 19.4 & 19.5 & 19.6 & & 0.04 & -40.5 & 24.8 & 25.6 & 7.5 & 0.13 & 0.76 & 0.21 & 19.0 & 19.2 & -8.6 \\
\hline $\mathrm{d} 0955+70$ & 18.5 & 18.6 & 18.8 & 1.03 & 0.04 & -2.3 & 26.6 & 27.1 & 18.6 & 0.32 & 0.61 & 0.43 & 17.5 & 17.9 & -9.9 \\
\hline $\mathrm{d} 0957+70$ & 19.5 & 19.3 & 19.9 & & 0.04 & 63.3 & 27.1 & 27.3 & 11.3 & 0.20 & 0.36 & 0.24 & 18.2 & 19.1 & -8.7 \\
\hline d0958+66 & 14.8 & 14.8 & 15.0 & 0.84 & 0.36 & 84.0 & 21.6 & 22.3 & 11.6 & 0.20 & 0.71 & 0.17 & 14.7 & 14.7 & -13. \\
\hline $\mathrm{d} 0959+68^{\dagger}$ & 16.4 & 16.5 & 16.2 & 0.74 & 0.10 & -54.0 & 26.0 & 25.5 & 26.6 & 0.46 & 0.21 & 0.20 & 16.2 & 16.2 & -11.6 \\
\hline $\mathrm{d} 1006+67$ & 18.3 & 18.5 & 18.1 & & 0.05 & -3.0 & 26.2 & 26.5 & 18.1 & 0.32 & 0.44 & 0.17 & 17.6 & 18.0 & -9.8 \\
\hline $\mathrm{d} 1009+70$ & 17.9 & 17.8 & 17.8 & 0.68 & 0.19 & 73.3 & 24.9 & 25.2 & 11.6 & 0.20 & 0.50 & 0.38 & 17.4 & 17.5 & -10.3 \\
\hline $\mathrm{d} 1012+64$ & 14.6 & 14.6 & 14.6 & & 0.15 & -72.1 & 21.5 & 22.3 & 13.9 & 0.24 & 0.88 & 0.05 & 14.5 & 14.5 & -13.3 \\
\hline $\mathrm{d} 1013+68^{\dagger}$ & 19.0 & 19.6 & 18.6 & & 0.10 & 56.4 & 26.9 & 27.9 & 29.5 & 0.51 & 1.00 & 0.11 & 18.5 & 18.5 & -9.3 \\
\hline $\mathrm{d} 1014+68$ & 18.7 & 18.8 & 18.8 & & 0.01 & 82.0 & 27.2 & 27.3 & 19.7 & 0.34 & 0.29 & 0.13 & 17.5 & 18.5 & -9.3 \\
\hline $\mathrm{d} 1015+69^{\dagger}$ & 19.4 & 19.5 & 19.4 & & 0.07 & 41.2 & 25.5 & 26.0 & 8.5 & 0.15 & 0.68 & 0.13 & 18.9 & 19.1 & -8.7 \\
\hline $\mathrm{d} 1016+69$ & 18.9 & 19.0 & 19.1 & & 0.01 & -1.5 & 27.1 & 27.6 & 19.3 & 0.34 & 0.60 & 0.13 & 17.8 & 18.4 & -9.4 \\
\hline $\mathrm{d} 1019+69$ & 18.1 & 17.5 & 18.1 & & 0.09 & -51.0 & 24.5 & 25.0 & 9.8 & 0.17 & 0.63 & 0.11 & 17.7 & 17.8 & -10.0 \\
\hline $\mathrm{d} 1020+69$ & 18.6 & 18.3 & 18.1 & & 0.13 & 59.4 & 26.7 & 26.9 & 22.3 & 0.39 & 0.41 & 0.10 & 17.5 & 18.1 & -9.7 \\
\hline $\mathrm{d} 1028+70$ & 15.7 & 15.7 & 15.7 & 0.77 & 0.20 & 36.1 & 22.4 & 23.3 & 13.4 & 0.23 & 0.88 & 0.11 & 15.5 & 15.5 & -12.3 \\
\hline $\mathrm{d} 1041+70$ & 18.5 & 18.6 & 19.0 & 1.00 & 0.18 & -63.1 & 26.1 & 26.2 & 10.9 & 0.19 & 0.30 & 0.15 & 18.1 & 18.5 & -9.3 \\
\hline $\mathrm{d} 1048+70^{\dagger}$ & 18.3 & 19.5 & 19.4 & & 0.02 & 80.3 & 27.3 & 27.6 & 17.5 & 0.30 & 0.42 & 0.10 & 18.2 & 18.7 & -9.1 \\
\hline
\end{tabular}

Note. - Measured apparent magnitudes and surface brightnesses are uncorrected for extinction.

${ }^{a}$ Magnitude measured in circular aperture, mean sky measured in circular annulus $>2 r_{\text {galaxy }}$

${ }^{\mathrm{b}}$ Aperture magnitude with sky+foreground contamination estimated from counts in nearby circular apertures

${ }^{\mathrm{c}}$ Magnitude from curve of growth fitting with cumulative Sersic function

${ }^{\mathrm{d}}$ Integrated color within $\mathrm{R}_{e}$

${ }^{\text {e} A v e r a g e ~ m e a s u r e d ~} r^{\prime}$ corrected to total magnitudes assuming an exponential profile, extinction corrected. See text. 
Table 4. Photometry of previously known M81 group members

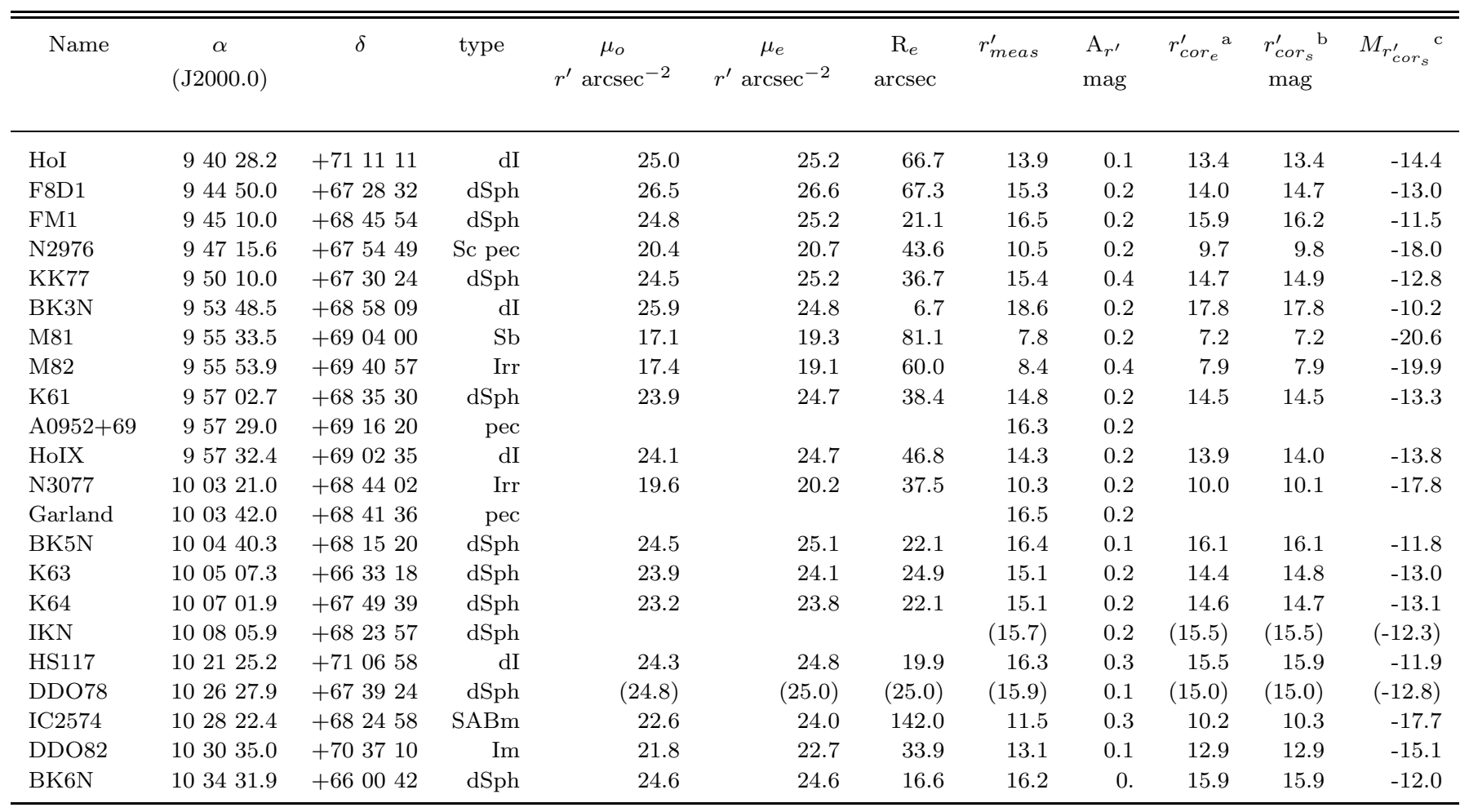

Note. - Values in parentheses highly uncertain due to presence of bright foreground stars. Values for brightest galaxies may be underestimates due to sky subtraction errors in the TERAPIX reduction pipeline.

${ }^{a}$ Corrected for extinction and flux lost in sky assuming an exponential profile.

${ }^{\mathrm{b}}$ Corrected for extinction and flux lost in sky assuming a Sersic profile.

${ }^{\mathrm{c}}$ Absolute Sersic profile corrected magnitude, using distance moduli from Table 2 of Karachentsev et al. (2002a) or assuming 27.8 where not provided.

Table 5. Faint-end slope from differential luminosity functions

\begin{tabular}{llrr}
\hline \hline \multicolumn{1}{c}{ Sample } & $\alpha\left(r_{\text {meas }}^{\prime}\right)$ & $\alpha\left(r_{\text {cor }(\text { exp })}^{\prime}\right)$ & $\alpha\left(r_{\text {cor }(\text { sersic })}^{\prime}\right)$ \\
& & & \\
\hline All (completeness corr. $)$ & $-1.29_{-0.04}^{+0.04}$ & $-1.28_{-0.05}^{+0.04}$ & $-1.32_{-0.05}^{+0.04}$ \\
All & $-1.26_{-0.08}^{+0.09}$ & $-1.39_{-0.05}^{+0.05}$ & $-1.36_{-0.08}^{+0.09}$ \\
Best & $-1.20_{-0.10}^{+0.10}$ & $-1.34_{-0.07}^{+0.07}$ & $-1.30_{-0.10}^{+0.10}$ \\
Original & $-1.19_{-0.07}^{+0.07}$ & $-1.24_{-0.08}^{+0.09}$ & $-1.26_{-0.08}^{+0.08}$ \\
\hline
\end{tabular}


Table 6. Faint-end slope from integrated luminosity functions

\begin{tabular}{llrr}
\hline \hline \multicolumn{1}{c}{ Sample } & $\alpha\left(r_{\text {meas }}^{\prime}\right)$ & $\alpha\left(r_{\text {cor }(\text { exp })}^{\prime}\right)$ & $\alpha\left(r_{\text {cor }(\text { sersic })}^{\prime}\right)$ \\
\hline All (completeness corr.) & $-1.29_{-0.05}^{+0.02}$ & $-1.28_{-0.05}^{+0.03}$ & $-1.28_{-0.05}^{+0.02}$ \\
All & $-1.29_{-0.06}^{+0.04}$ & $-1.29_{-0.08}^{+0.05}$ & $-1.28_{-0.05}^{+0.04}$ \\
Best & $-1.27_{-0.06}^{+0.05}$ & $-1.27_{-0.07}^{+0.04}$ & $-1.26_{-0.05}^{+0.05}$ \\
Original & $-1.26_{-0.10}^{+0.07}$ & $-1.24_{-0.07}^{+0.06}$ & $-1.25_{-0.06}^{+0.06}$ \\
\hline
\end{tabular}




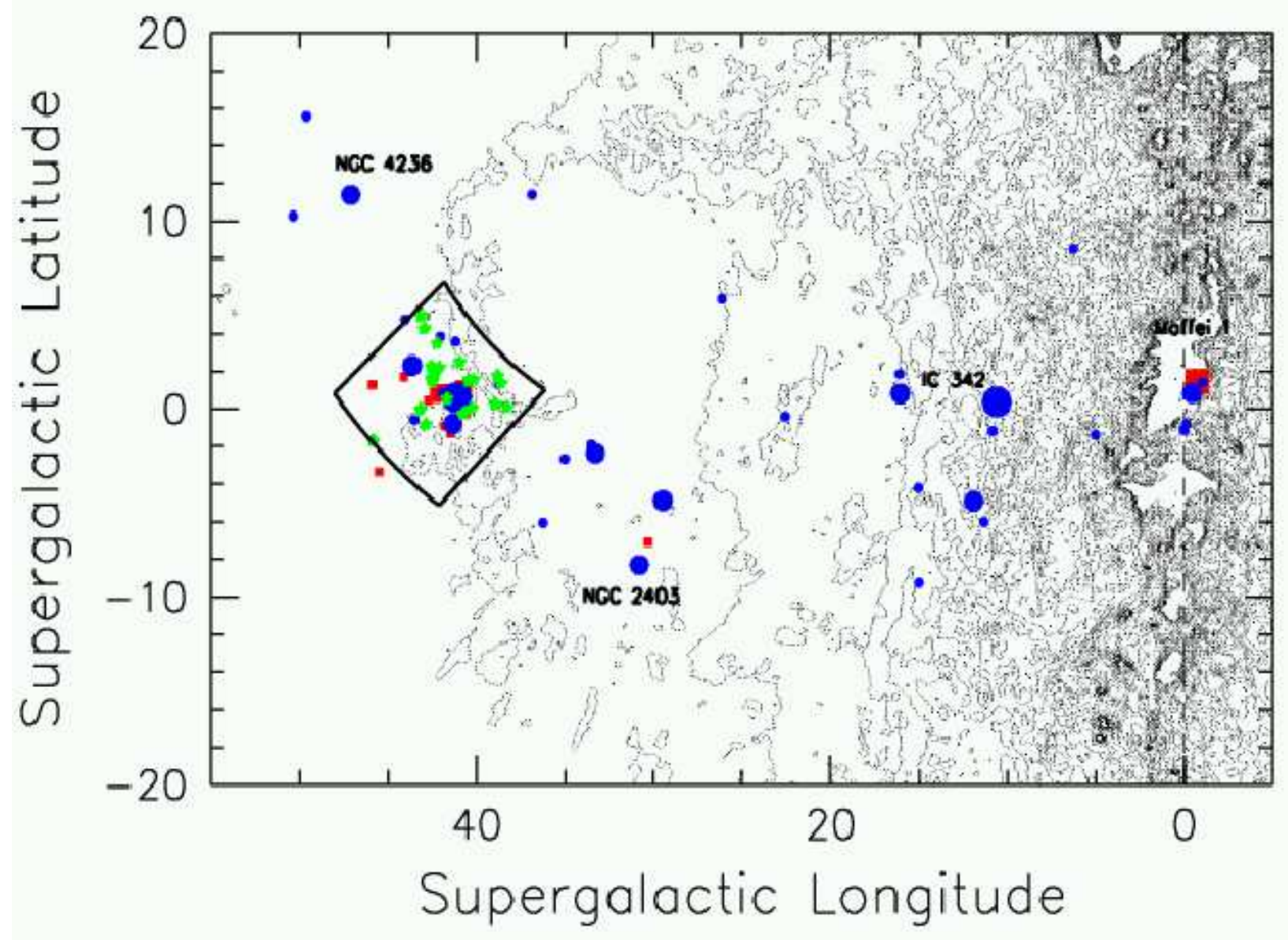

Fig. 1.- The extended region around M81. All galaxies with velocities in the Galactic standard of rest less than $400 \mathrm{~km} \mathrm{~s}^{-1}$ in an area of $60 \times 40$ degrees in supergalactic coordinates. Circles: spirals and irregulars. Squares: early types. Large, mid, and small symbols identify galaxies with $M_{B}<-20,-20<M_{B}<-16$, and $M_{B}>-16$, respectively. The box outlines the area of the CFHT MegaCam survey and the small stars identify the newly discovered dwarfs. Contours come from the dust maps of Schlegel et al. (1998), for every 0.2 $r^{\prime}$ magnitudes of extinction. 


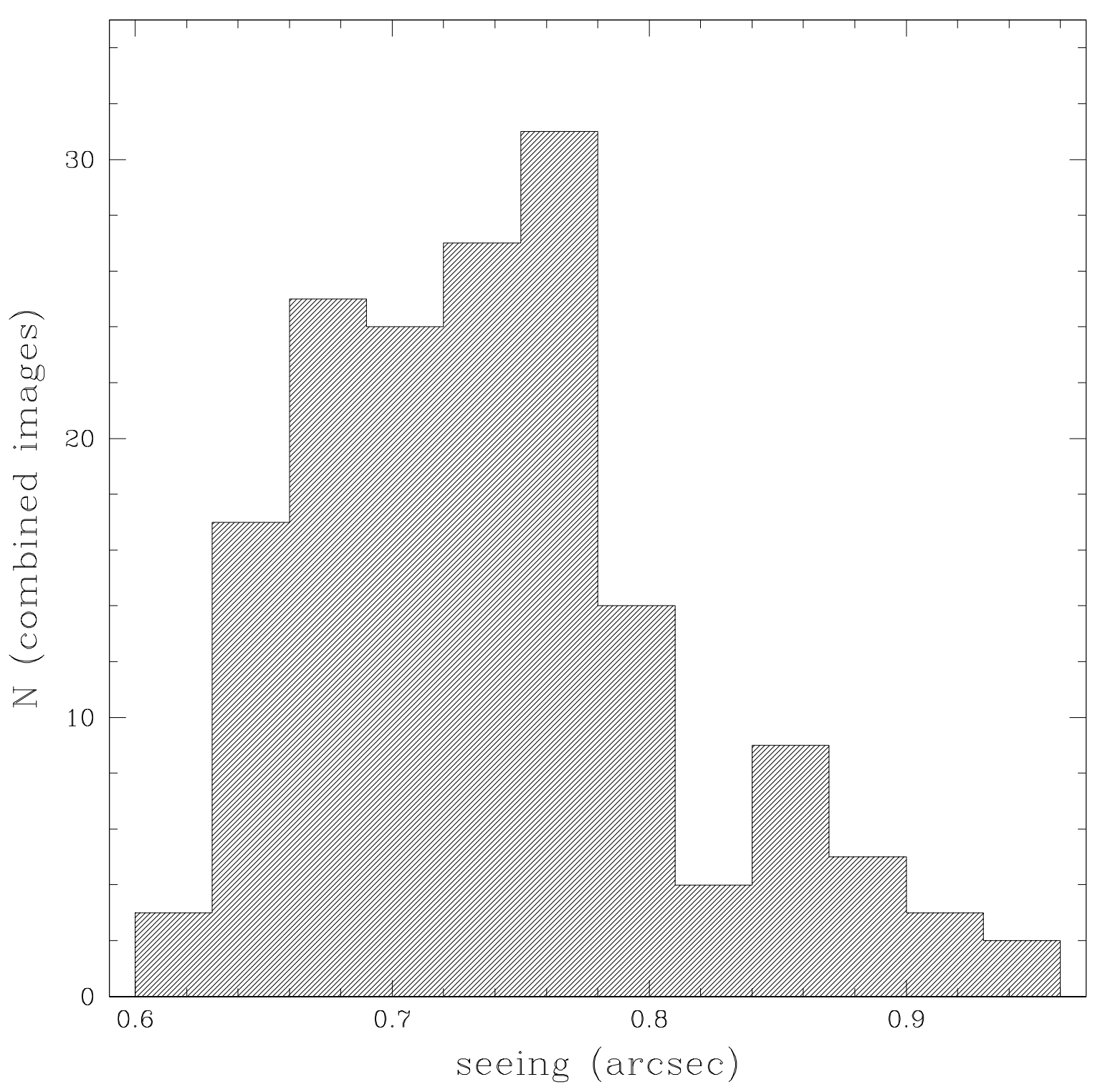

Fig. 2.- Histogram displaying the seeing range for all combined images in our survey. 


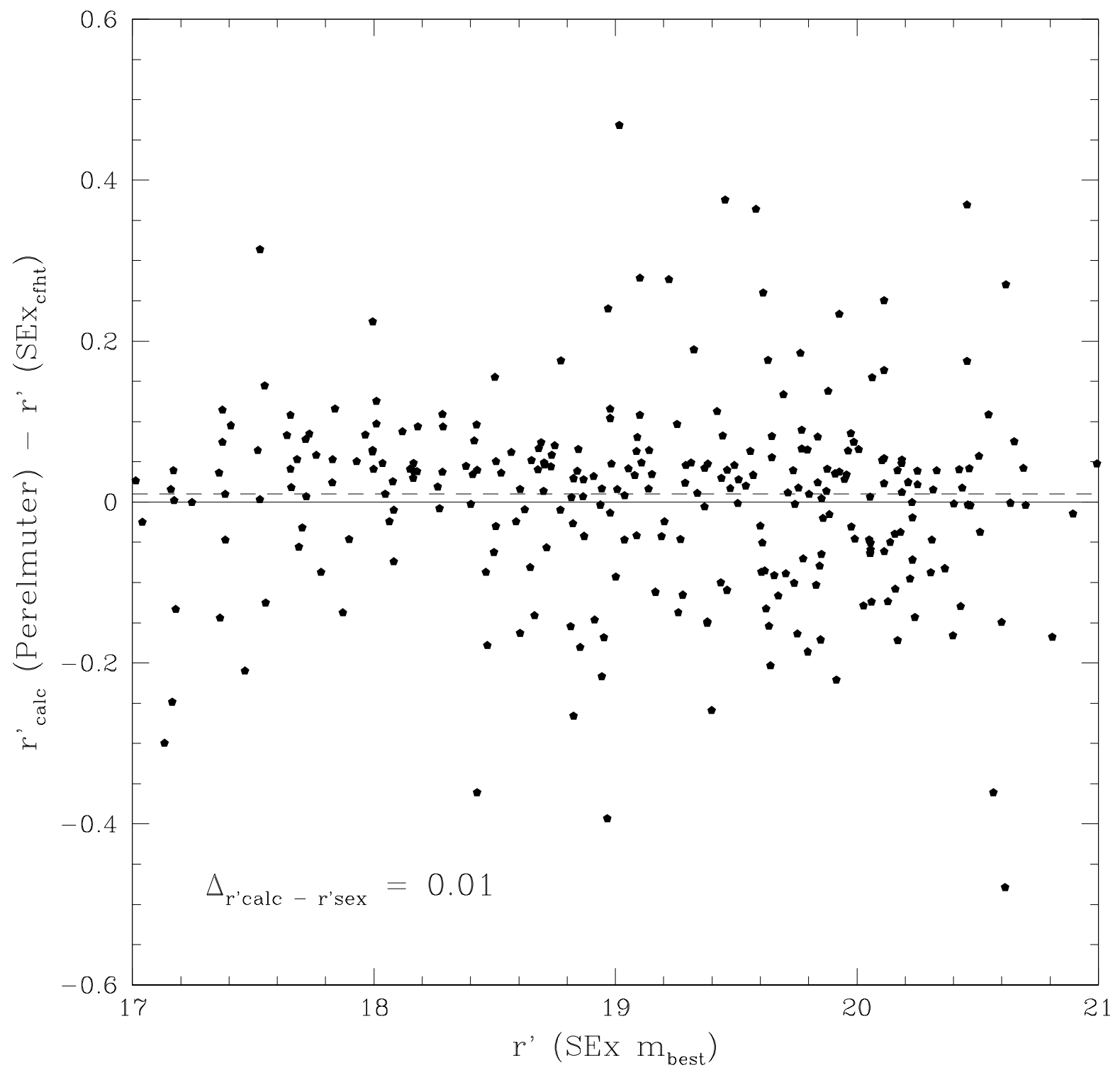

Fig. 3.- Tests of photometric accuracy. We compare our r' total magnitudes to transformed R magnitudes of Perelemuter \& Racine (1995). 

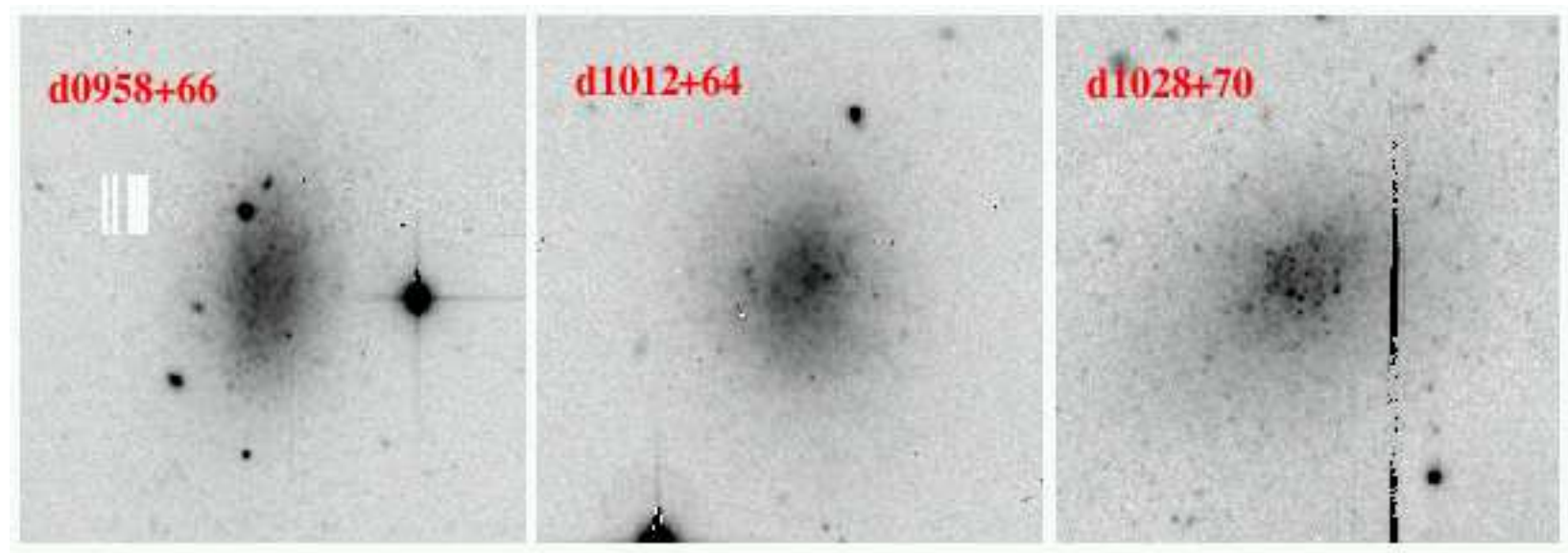

Fig. 4. - Thumbnails of the 3 new M81 candidate BCDs. Images are 1.5 arcmin on a side. 


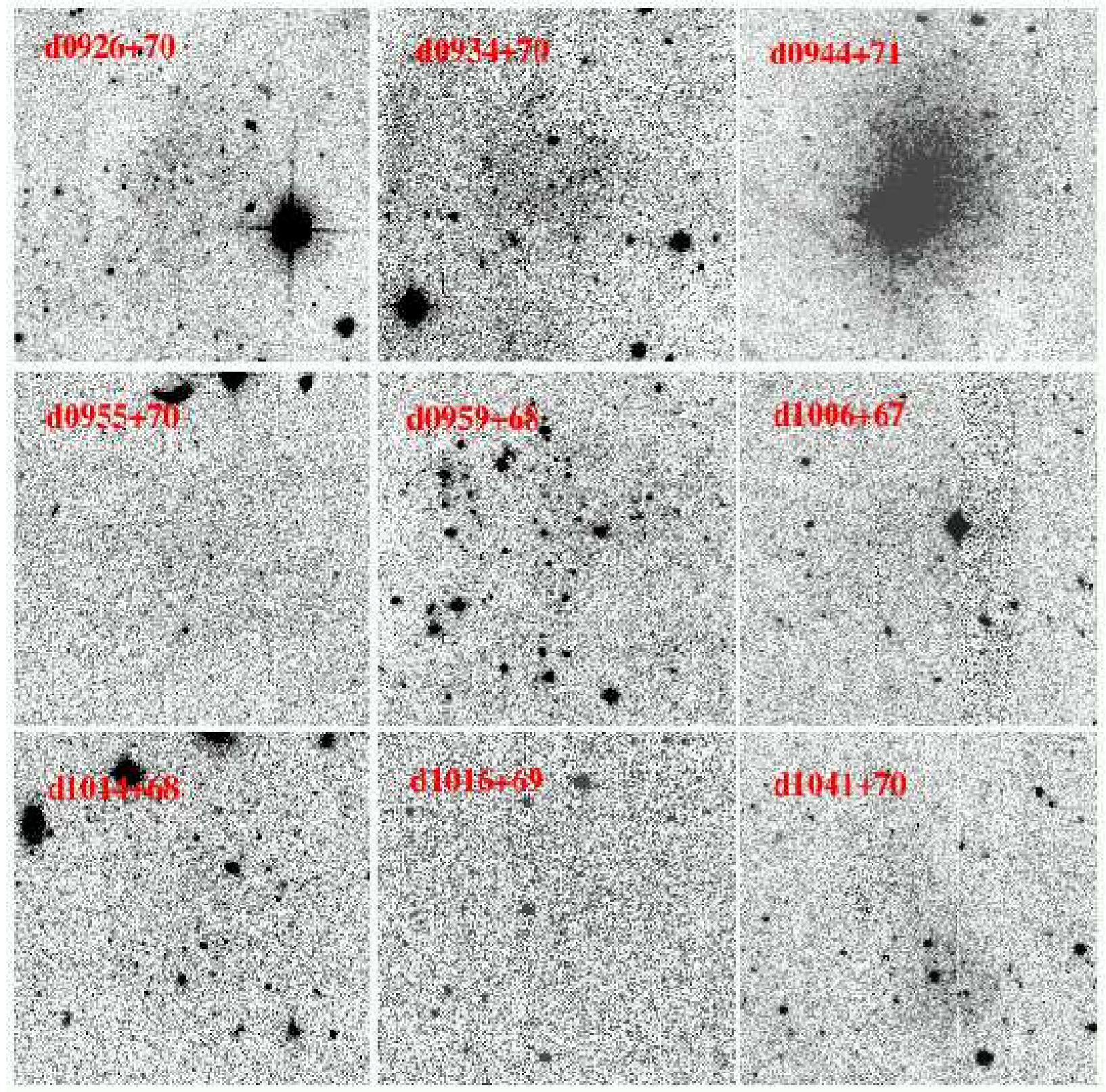

Fig. 5.- We display a mosaic of images of 9 new candidates that were detected by eye and/or 2-point correlation of resolved points. Images are 1.5 arcmin on a side. 


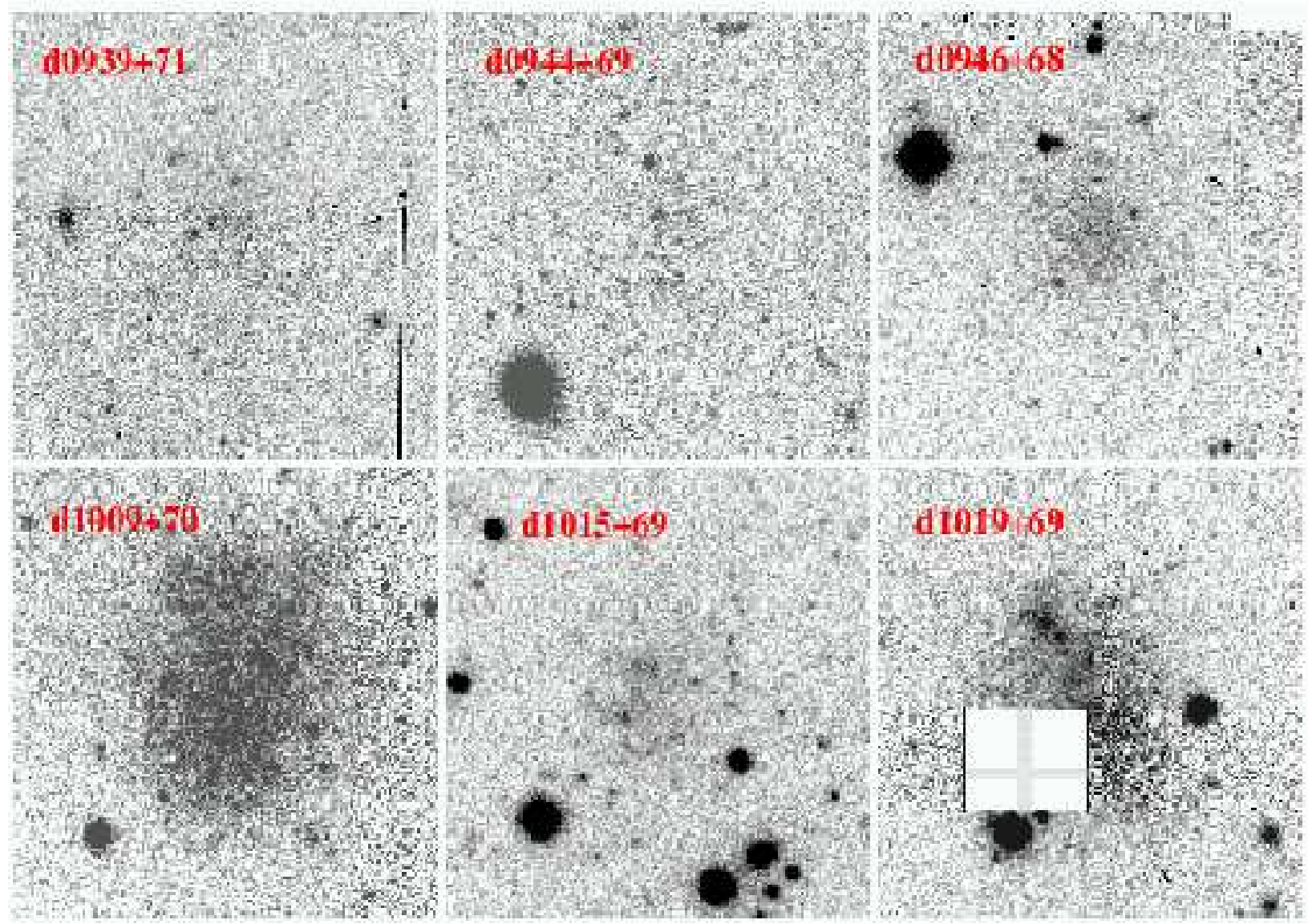

Fig. 6.- While these six candidates have a slight degree of resolution, many of these may prove to lie in the background of the M81 group.

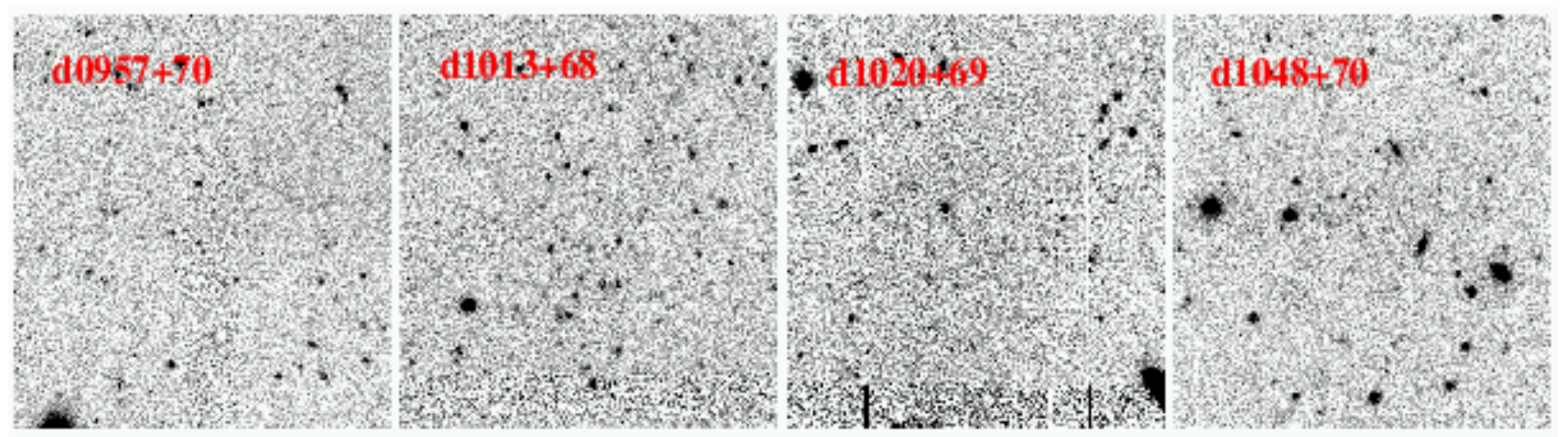

Fig. 7.- Four candidates which may turn out to be artifacts (excess noise or foreground concentrations of stars) or distant galaxy clusters. 


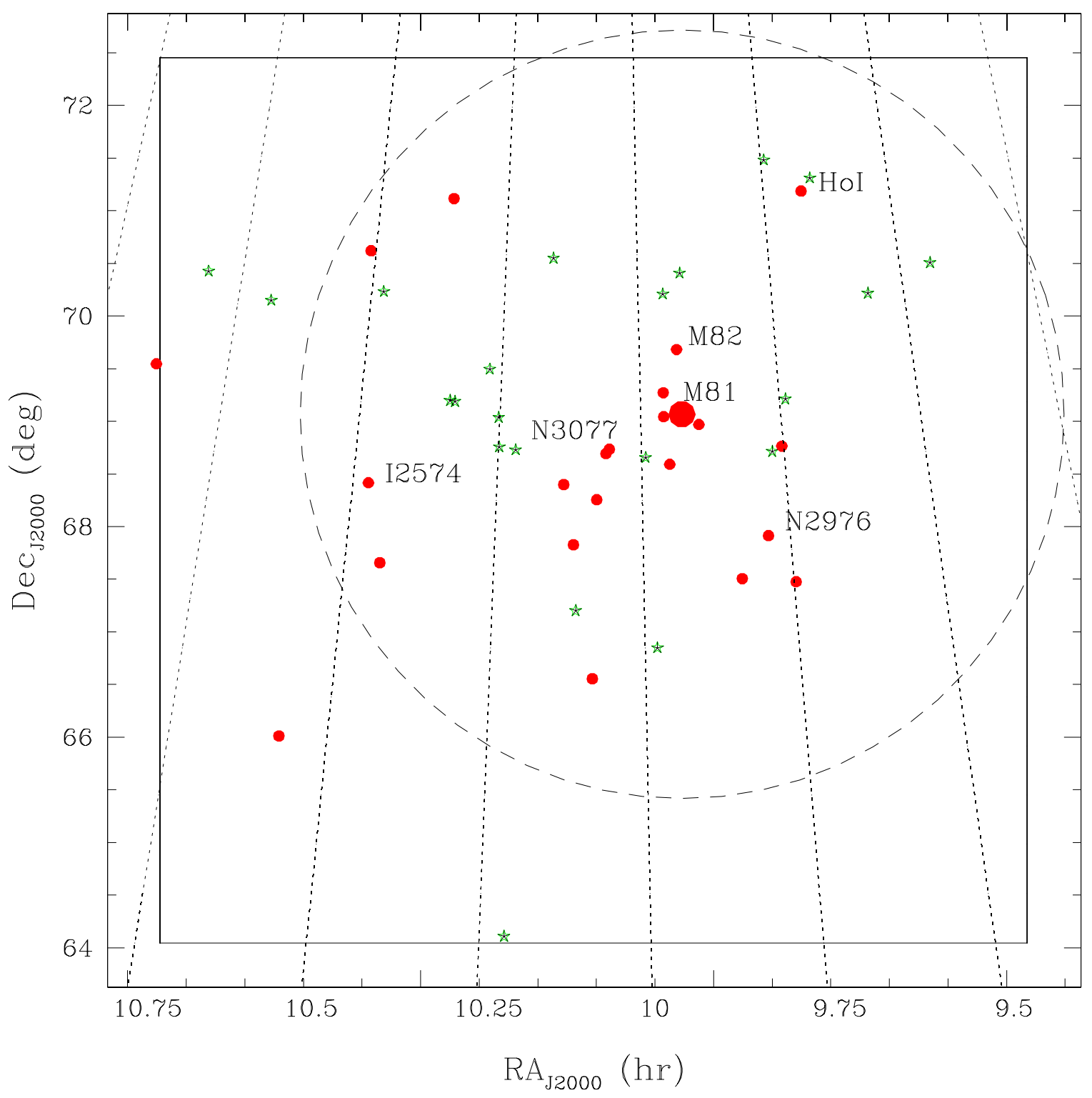

Fig. 8. - Map of our M81 survey region (large box almost filling the figure). Circles indicate previously known group members while stars denote the location of the new group candidates. The large dashed circle is the projection of the putative surface of second turnaround at 230 kpc from M81. 


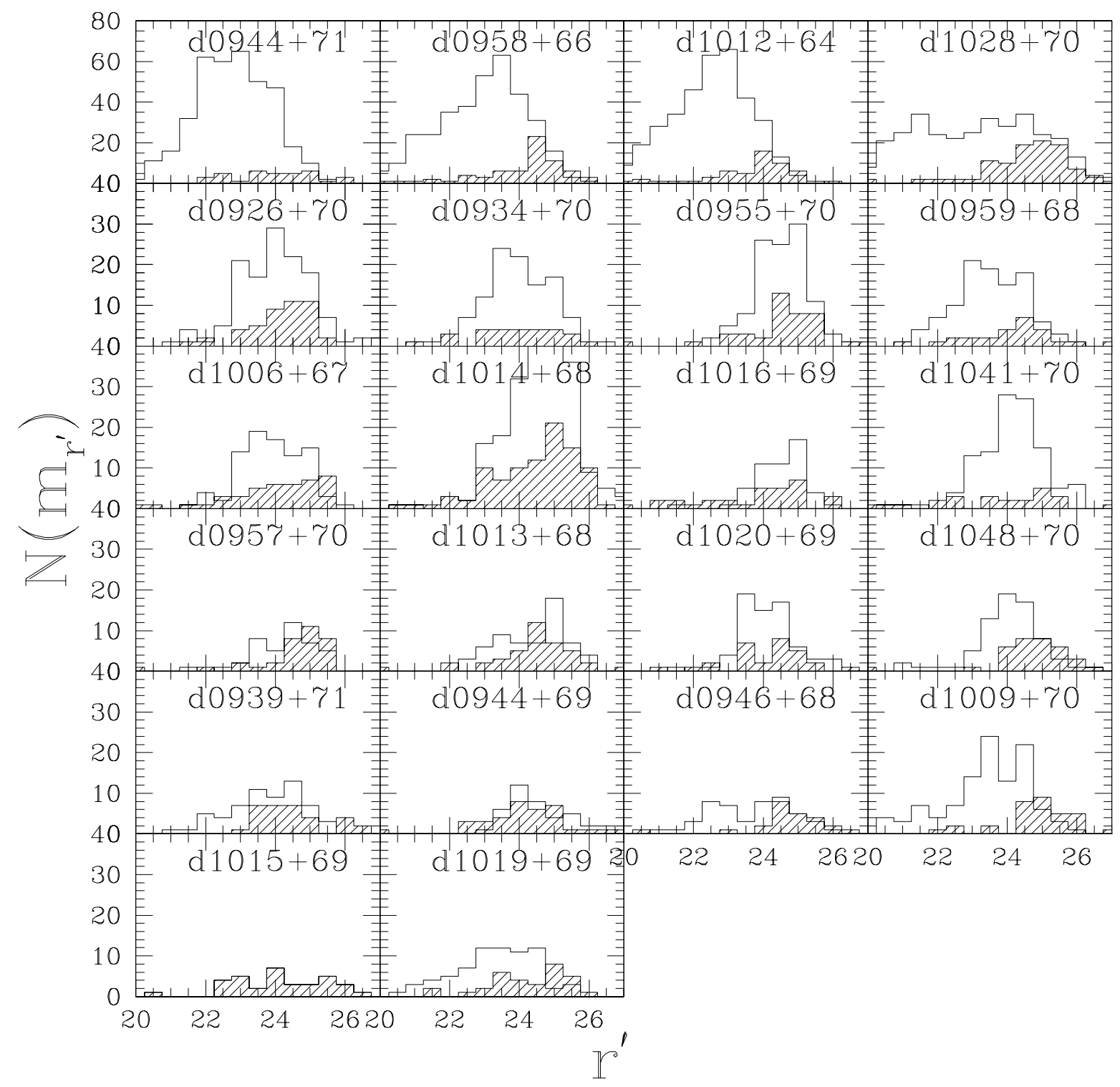

Fig. 9. - We display histograms of the resolved objects in the new M81 candidates. Number counts of objects recovered by SExtractor within a 30-45 arcsec radius (see text) centered on the new candidates are binned as a function of magnitude. The top row includes 4 objects, including $3 \mathrm{BCD}$ candidates, with the largest resolved stellar populations. The next 2 rows include a further 8 good candidates. The 4 th row contains 4 objects that may prove to be artifacts, while the bottom 2 rows include possible more distant galaxies. The shaded histograms represent number counts in an identical sized region 2.5 arcmin west of each galaxy. 

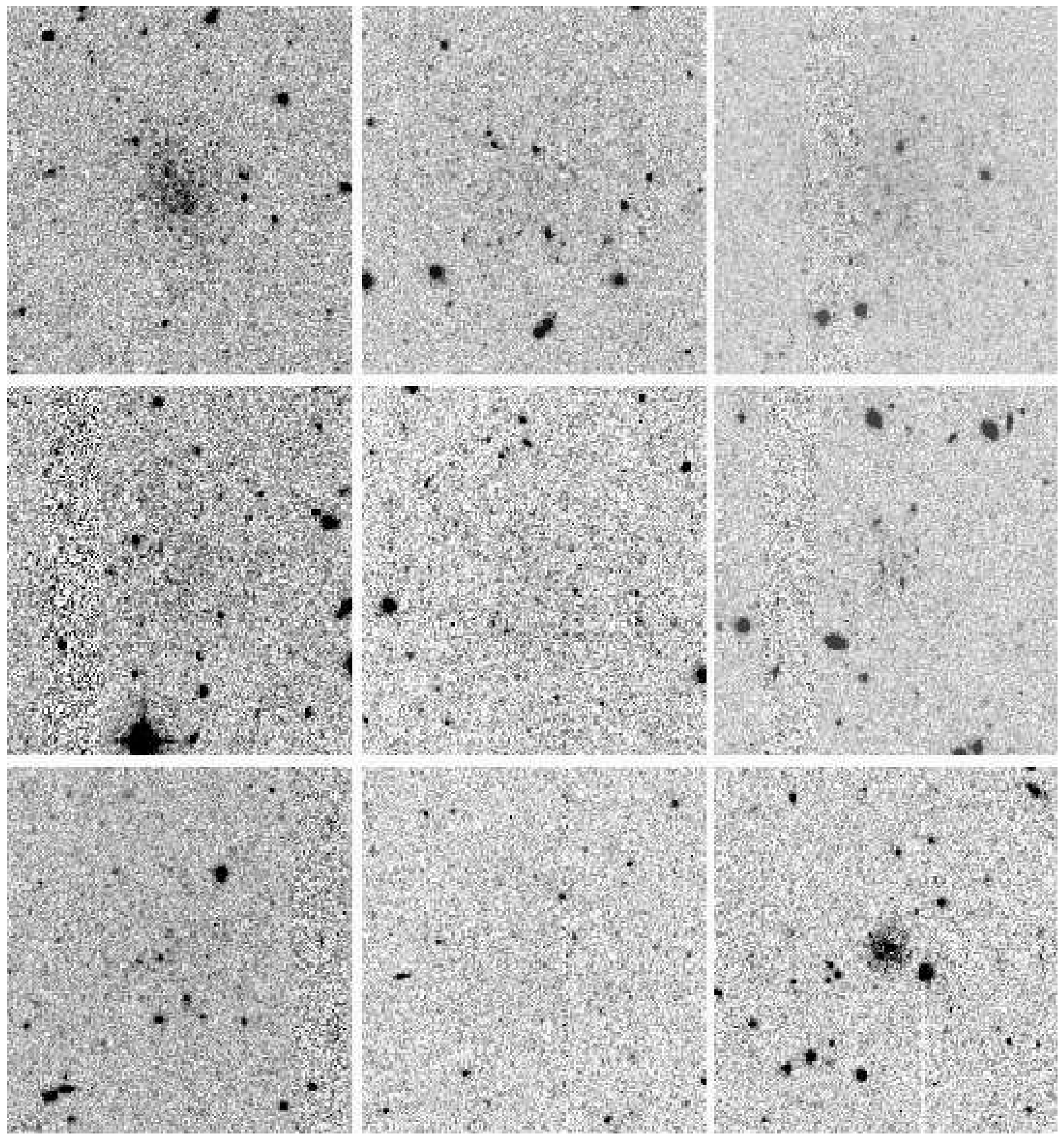

Fig. 10.- Examples of artificial galaxies that were added to real images with which we test the recovery efficiency of our detection techniques. Images are 1.5 arcmin on a side. 


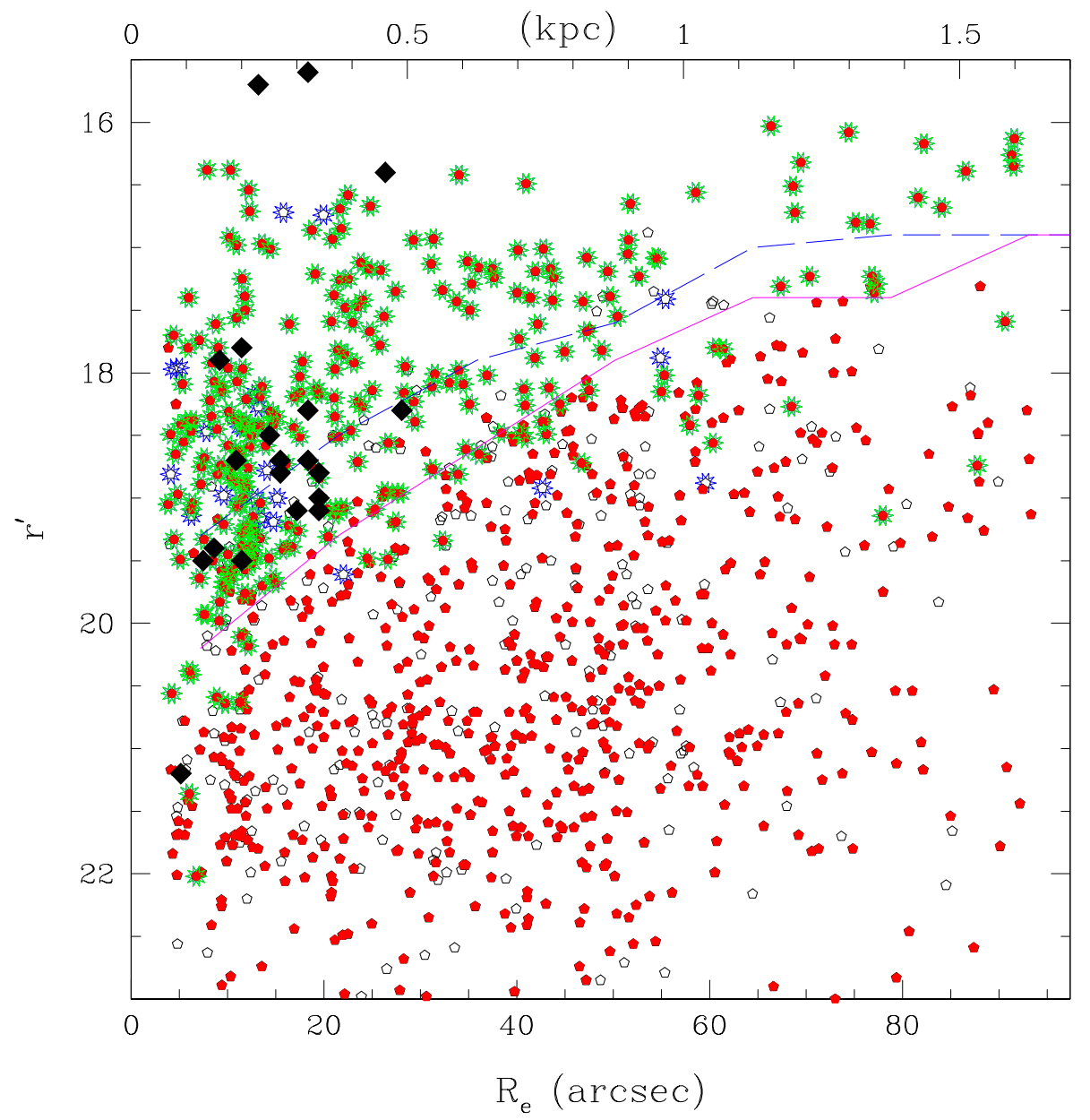

Fig. 11.- Results of 1200 artificial galaxy recovery tests. Small pentagons represent all artificial galaxies. Open pentagons are those galaxies that landed at least partially in chip gap regions of the MegaCam field or are largely obscured by bright saturated stars or other objects and are less likely to be recovered. Stars around these points denote that the simulated galaxy was recovered. Overlaid as black diamonds are the candidate real galaxies in this magnitude - effective radius plane. The solid line denotes the magnitude at which the recovery drops to $50 \%$ (in 2-magnitude bins), while the dashed line marks the boundary of $90 \%$ completeness. 


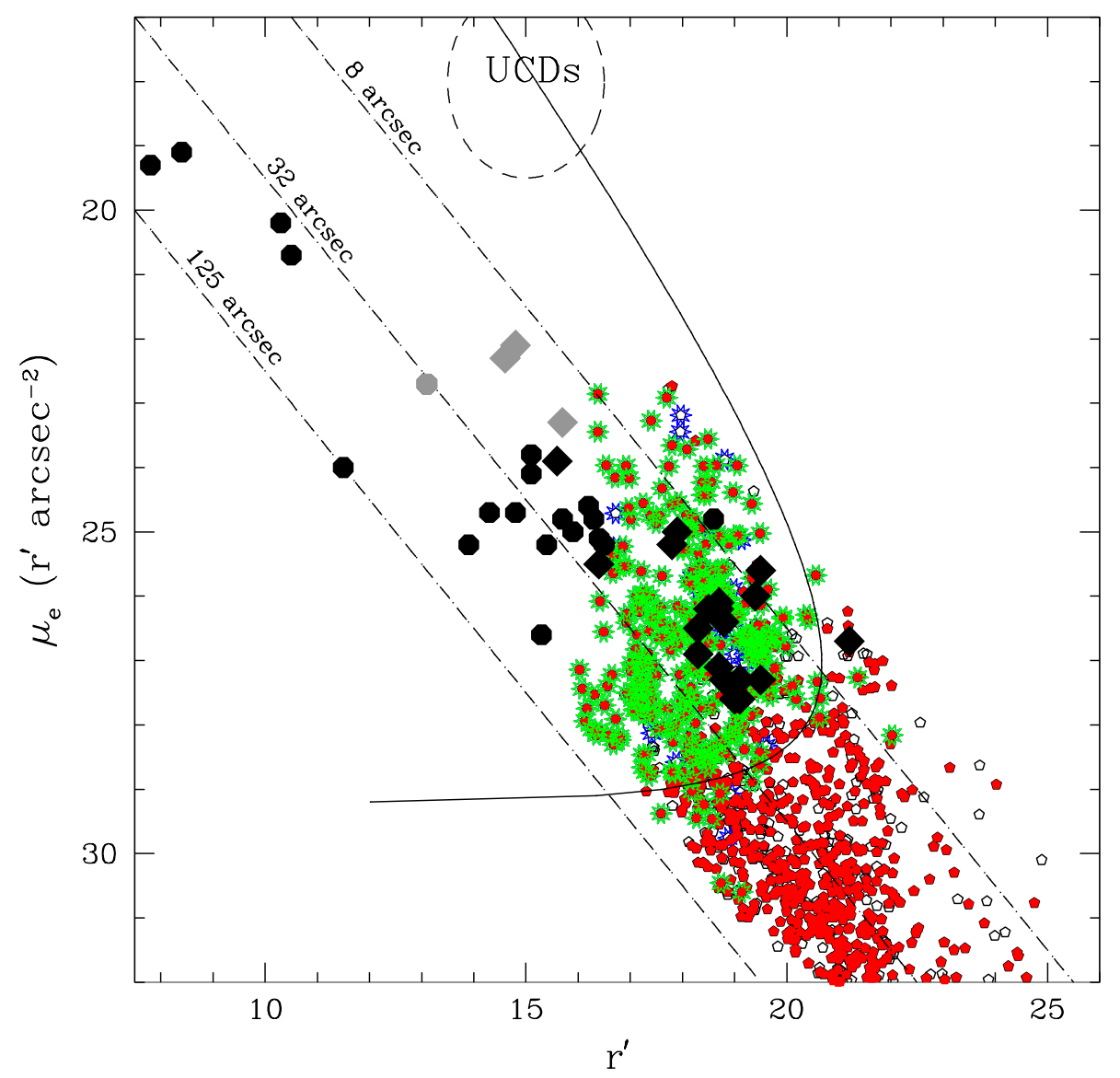

Fig. 12.- We plot the recovery of 1200 artificial galaxies in the magnitude-surface brightness plane. As before, pentagons represent all artificial galaxies. Green and blue stars around these points indicate that the simulated galaxy was recovered. Overlaid as black (gray) diamonds are the candidate real galaxies (BCDs) in this magnitude - effective surface brightness plane. Circles represent previously known M81 group galaxies. DDO 82, shaded in gray, is a potential BCD. Lines of constant effective radius for exponential profiles are labelled. We draw a solid curved line which best bounds the region above which the artificial galaxies are recovered. 


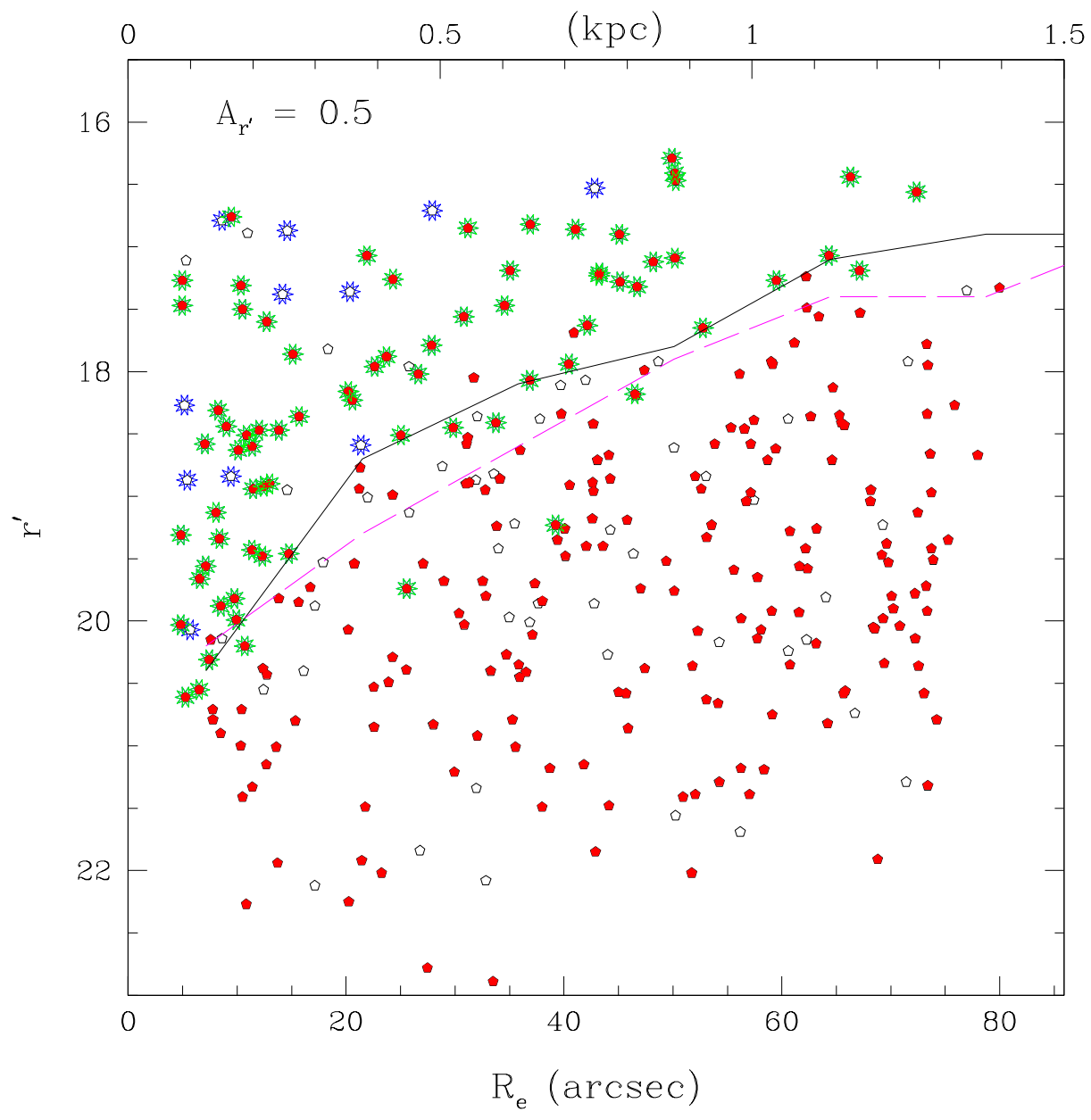

Fig. 13.- Recovery results for objects simulated with 0.5 magnitudes of extinction. Symbols as in previous figures. The solid line represents the magnitude at which the recovery drops to $50 \%$ for these galaxies, while the dashed line denotes the magnitude at which the recovery for artificial galaxies with 0.-0.2 magnitudes of extinction drops to $50 \%$. 


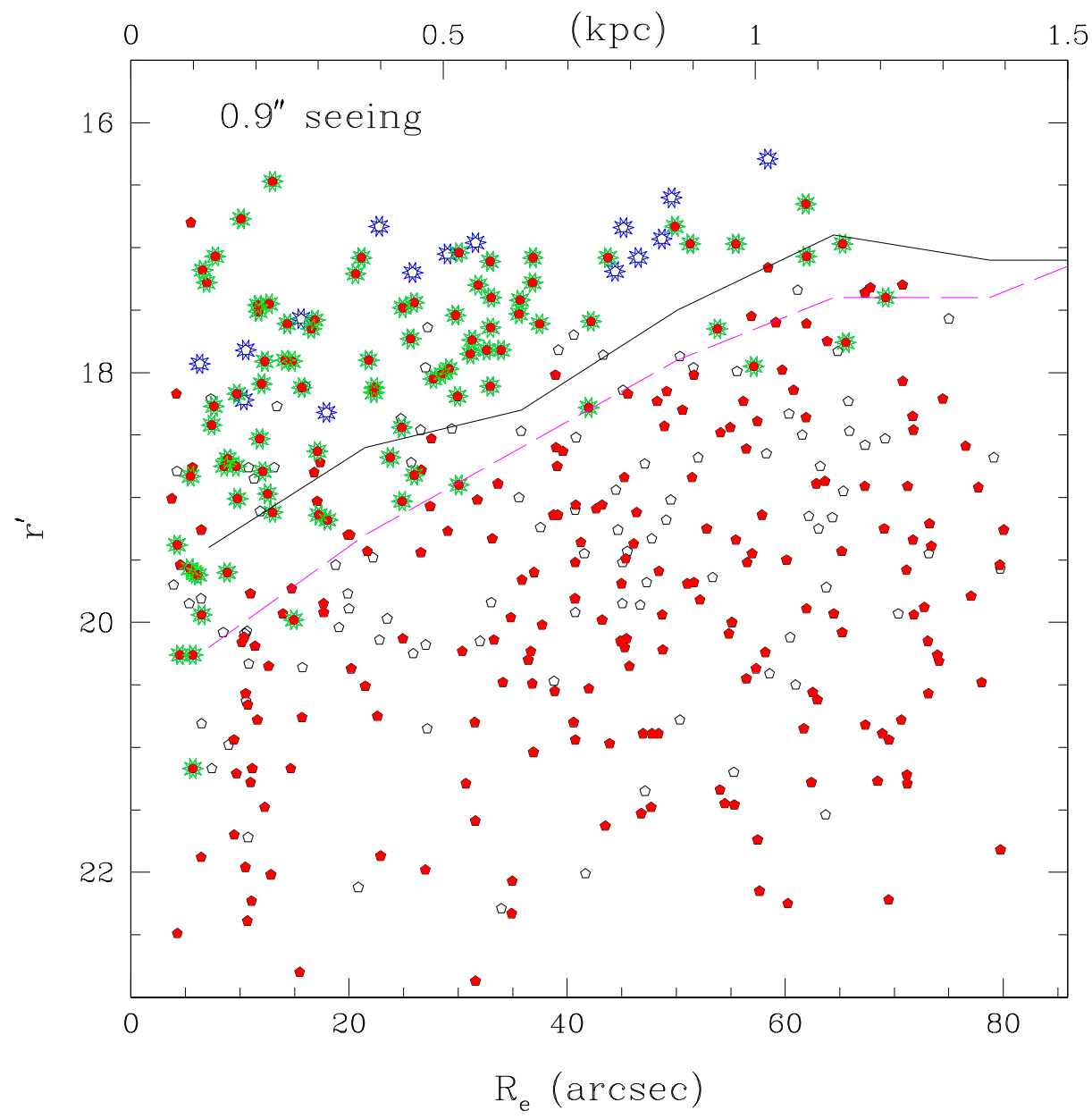

Fig. 14.- Recovery of 400 galaxies added with 0.9 arcsec seeing. Symbols as in previous figures. We compare results to that of the original 1200 simulations. The dashed line represents the magnitude at which the completeness drops to $50 \%$ for artificial galaxies added with seeing less that 0.75 arcsec. The solid line displays the $50 \%$ completeness for artificial galaxies added with 0.9 arcsec seeing. 


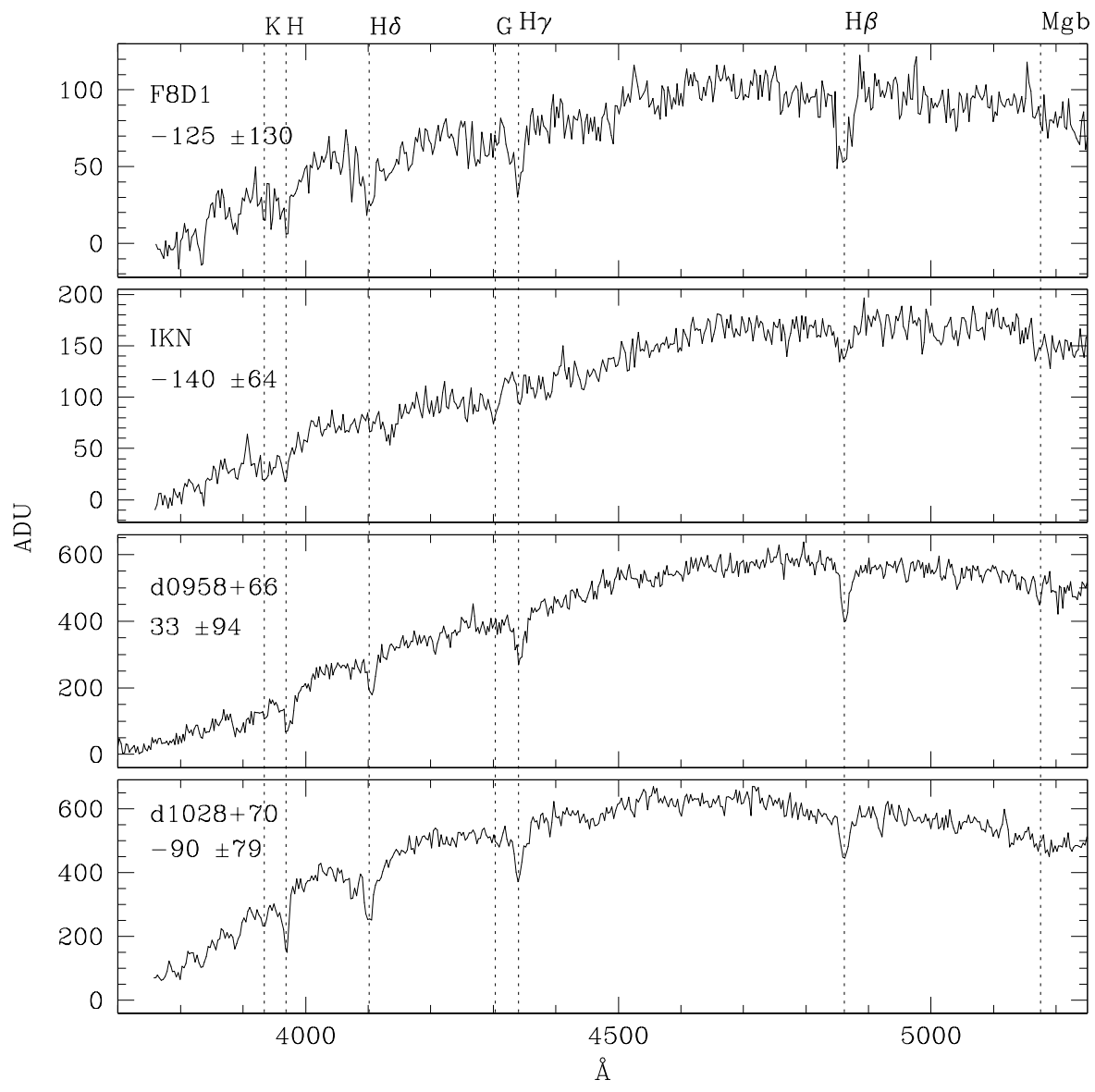

Fig. 15. - Spectra of 4 M81 group members obtained with Subaru/FOCAS. All spectra are shifted to the rest frame given the cross-correlation measured velocities as labelled. 


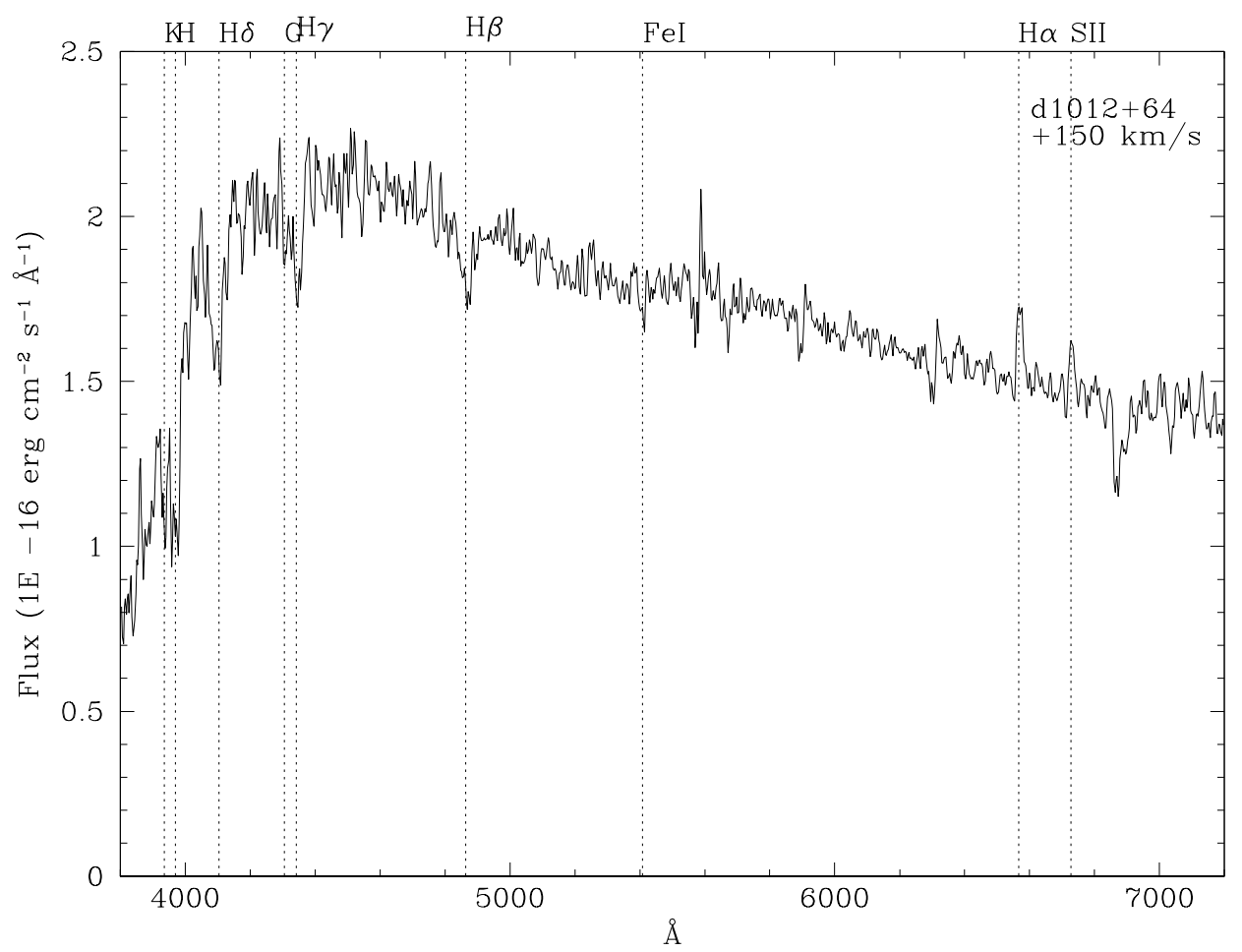

Fig. 16. - Spectrum of $\mathrm{d} 1012+64$ from the 6m BTA telescope. H $\alpha$ emission is detected and used to measure a heliocentric radial velocity. 


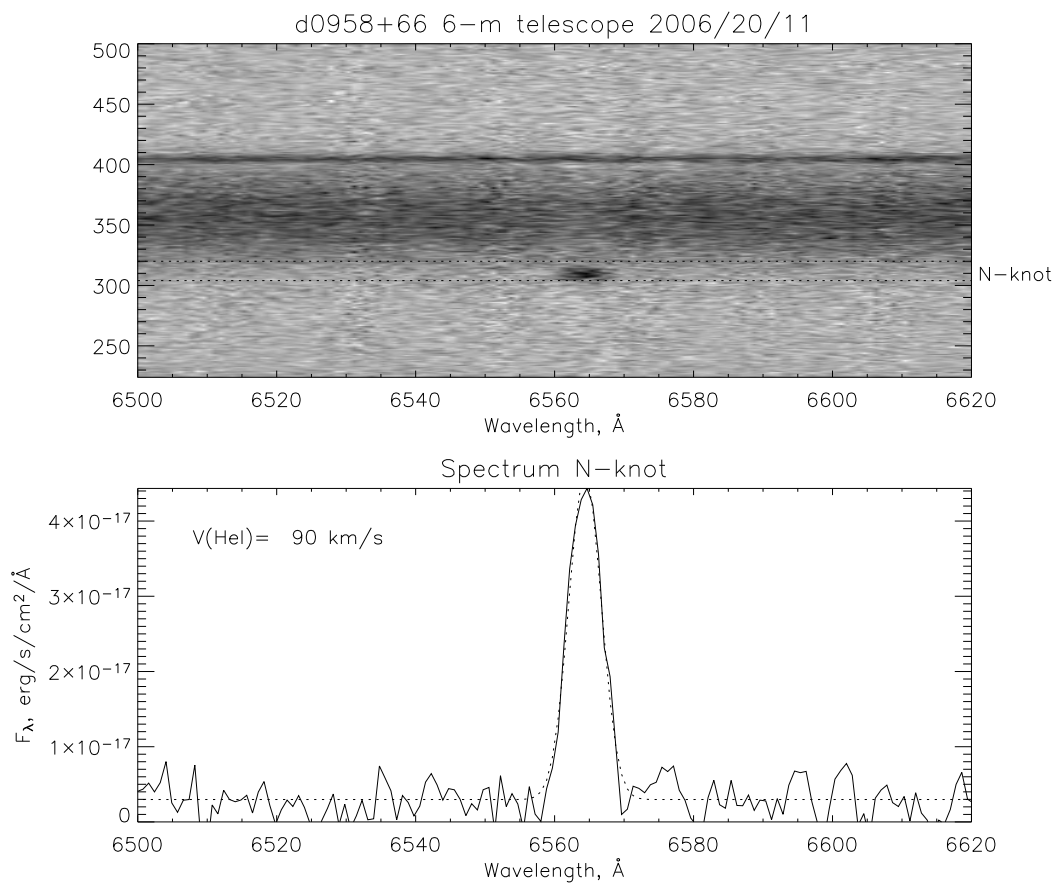

Fig. 17.- Spectrum of d0958+66 from the 6m BTA telescope. Strong $\mathrm{H} \alpha$ emission is detected and used to measure a heliocentric radial velocity. 

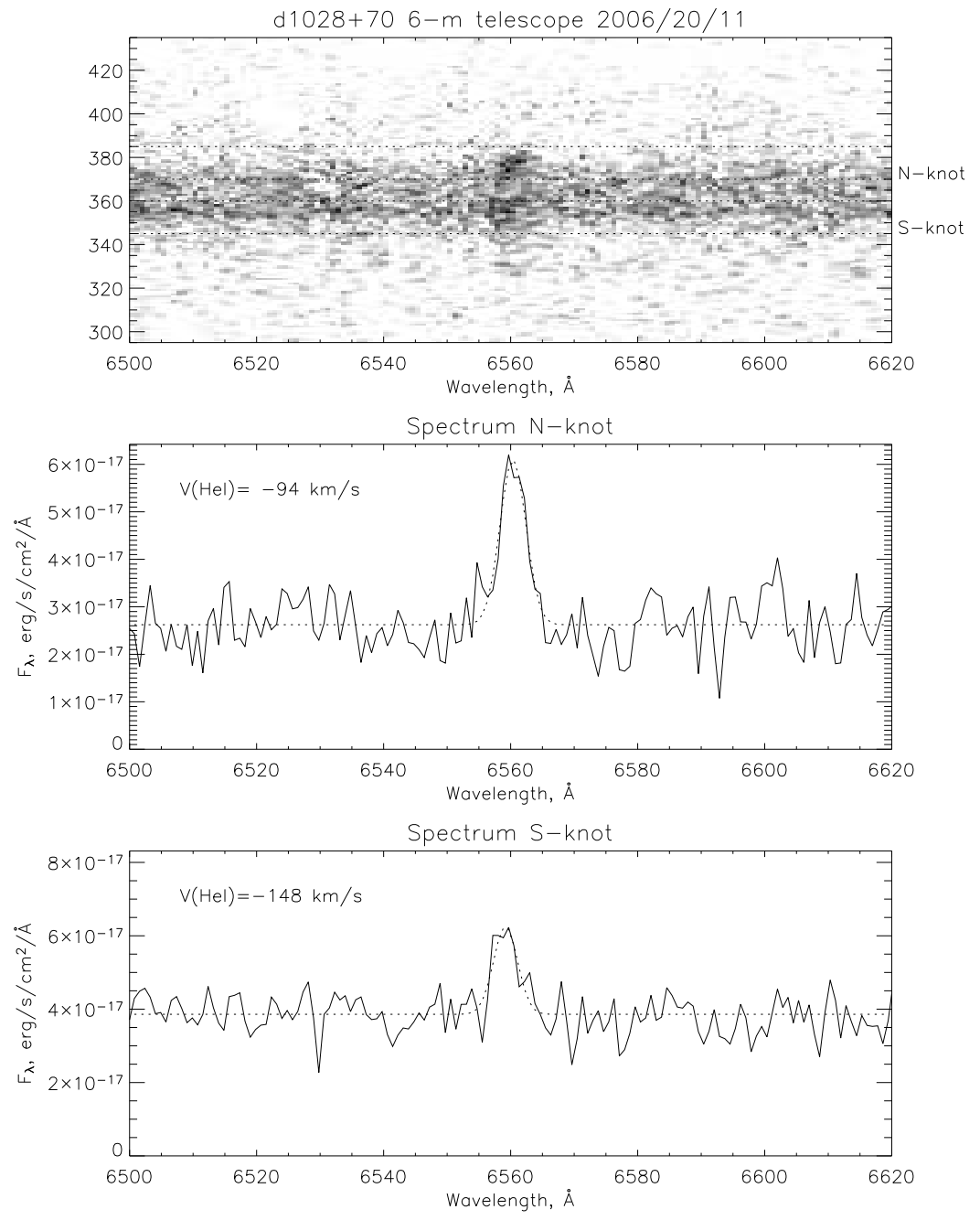

Fig. 18. - Spectrum of $\mathrm{d} 1028+70$ from the $6 \mathrm{~m}$ BTA telescope. $\mathrm{H} \alpha$ emission is detected in two separate knots which we label as $\mathrm{N}$ and S-knots. 


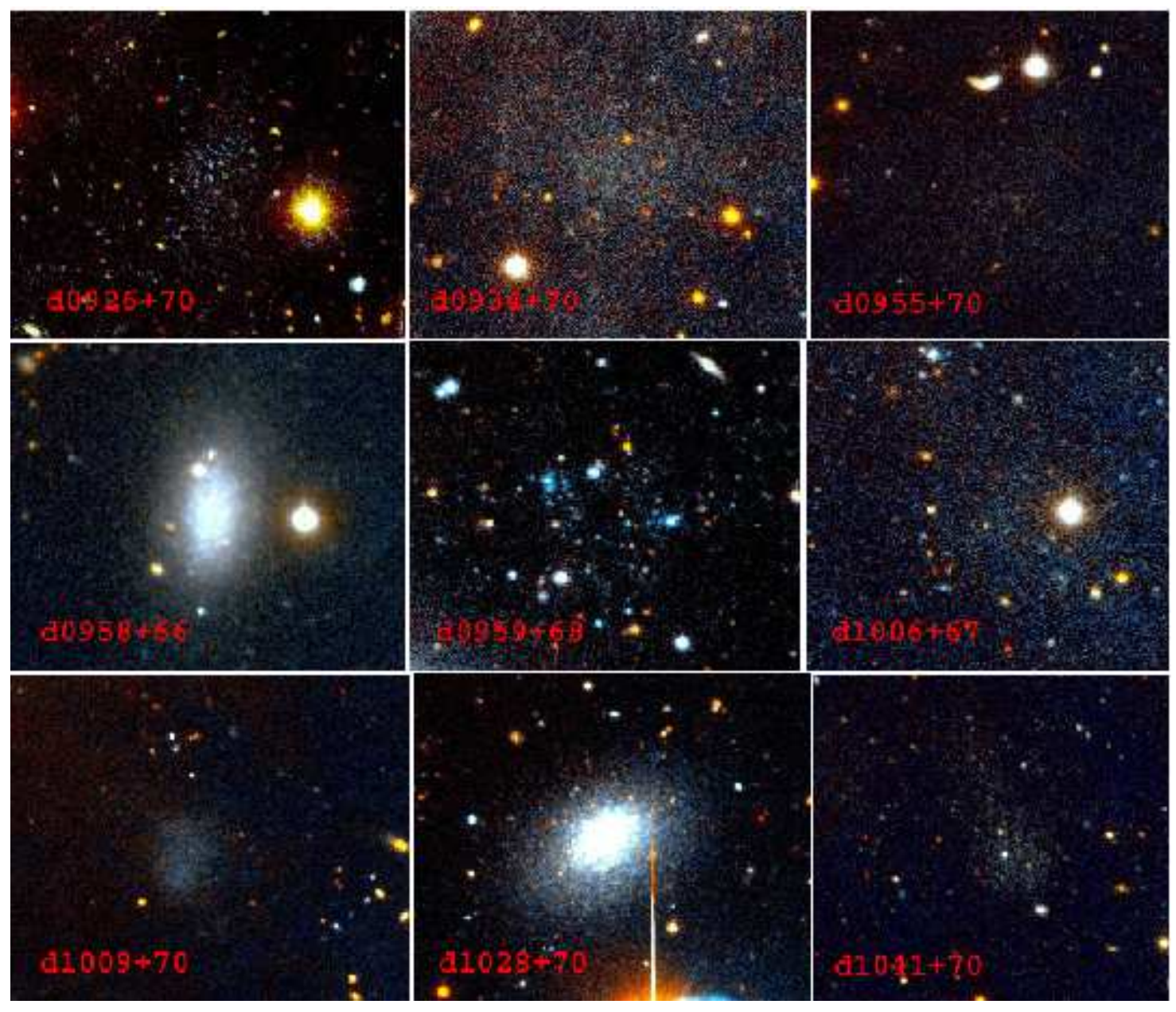

Fig. 19. - Color images based on 2 color photometry of 9 dwarf candidates. 


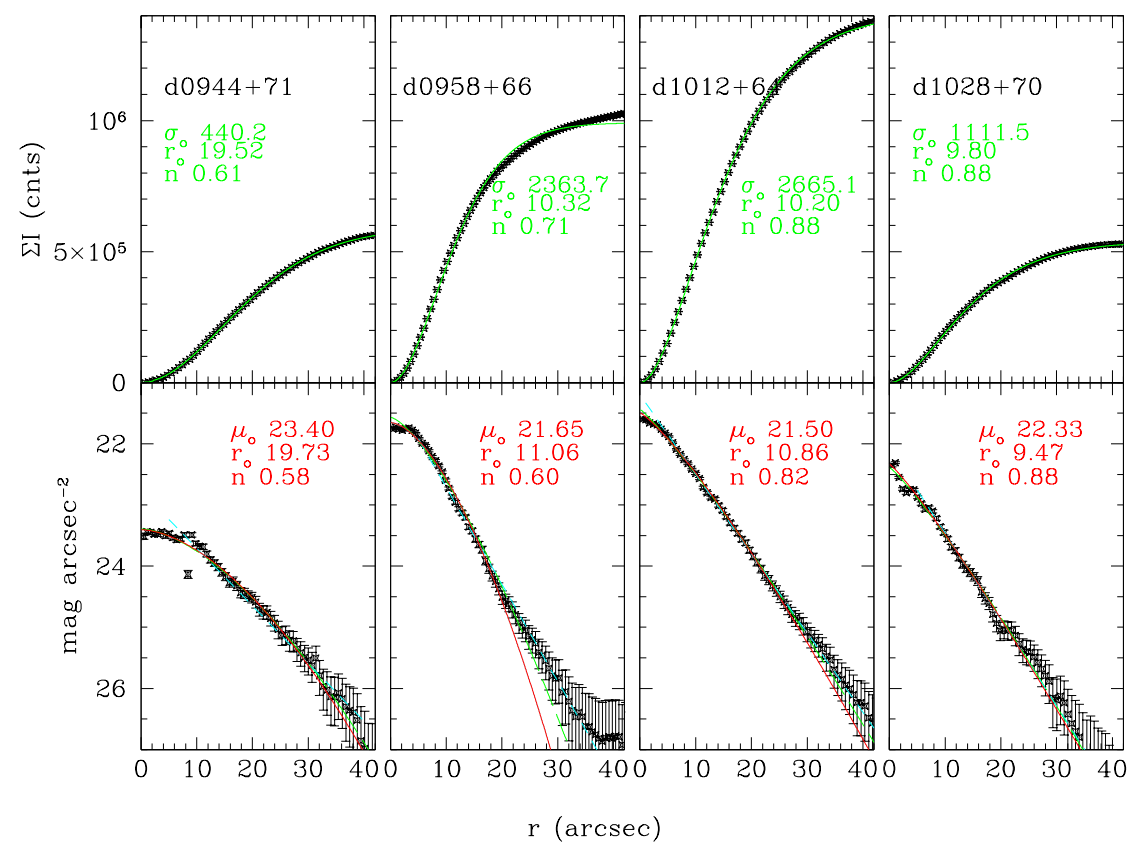

Fig. 20.- Curve of growth (top) and surface brightness profiles (bottom) for our 4 brightest candidates with best cumulative (top) and surface brightness profile (bottom) Sersic function fits overlaid. In the bottom plots, we also overlay the best cumulative Sersic function fit (green long dash) and best exponential surface brightness profile fit to outer radii only (cyan short dash). 


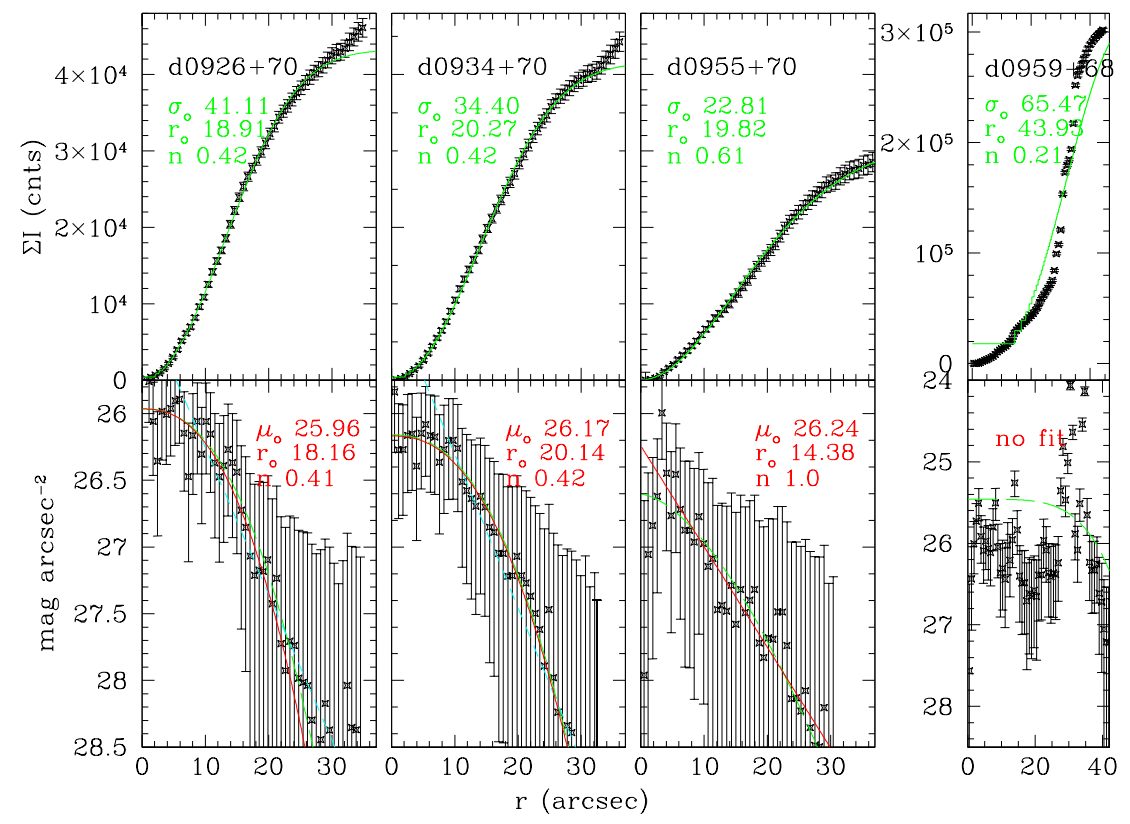

Fig. 21. - Fits for 4 of our candidate galaxies, as in Figure 20, Curve of growth profile data points which exhibit a sharp increase in slope beyond 10 arcsec were truncated during profile fitting. For d0926 +70 and d0934 +70 this rise was determined to be due to nearby bright cirrus. For d0959+68 we were unable to obtain a surface brightness profile fit due to the fact that this object consists almost entirely of bright stars without a significant low surface brightness component. 


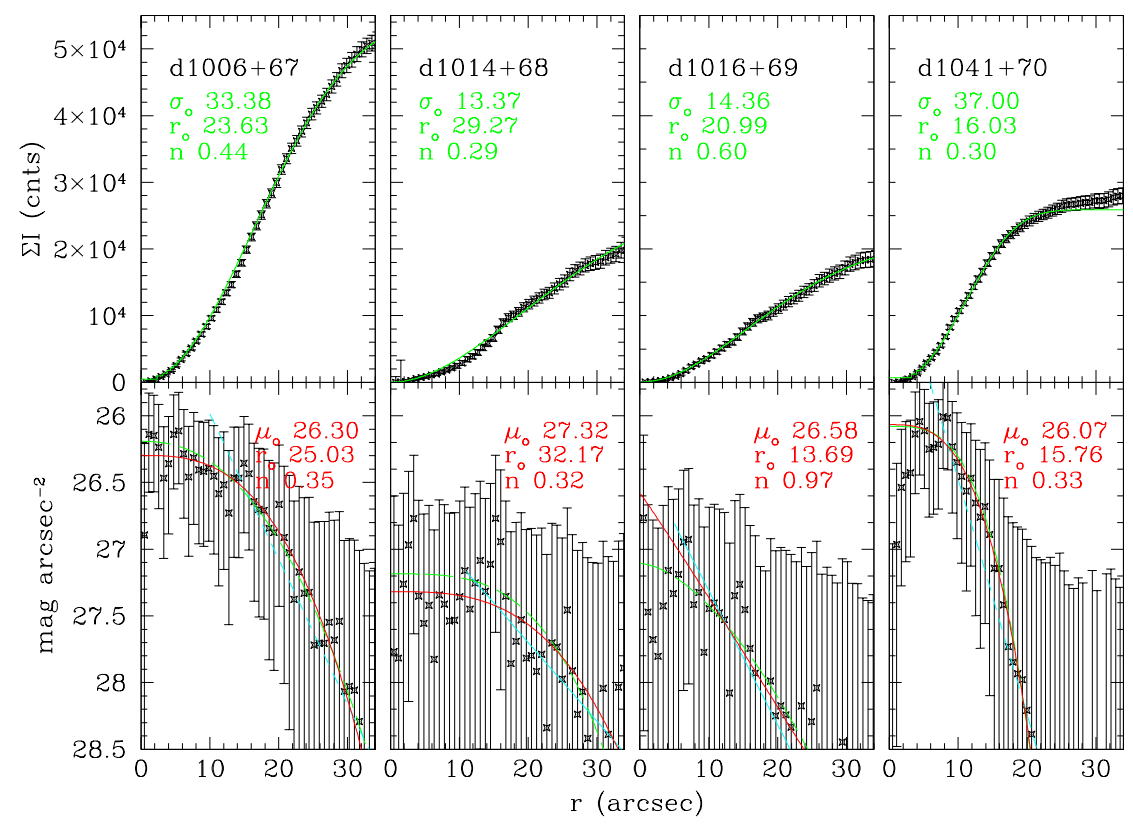

Fig. 22.- Fits for 4 of our candidate galaxies, as in Figure 20.

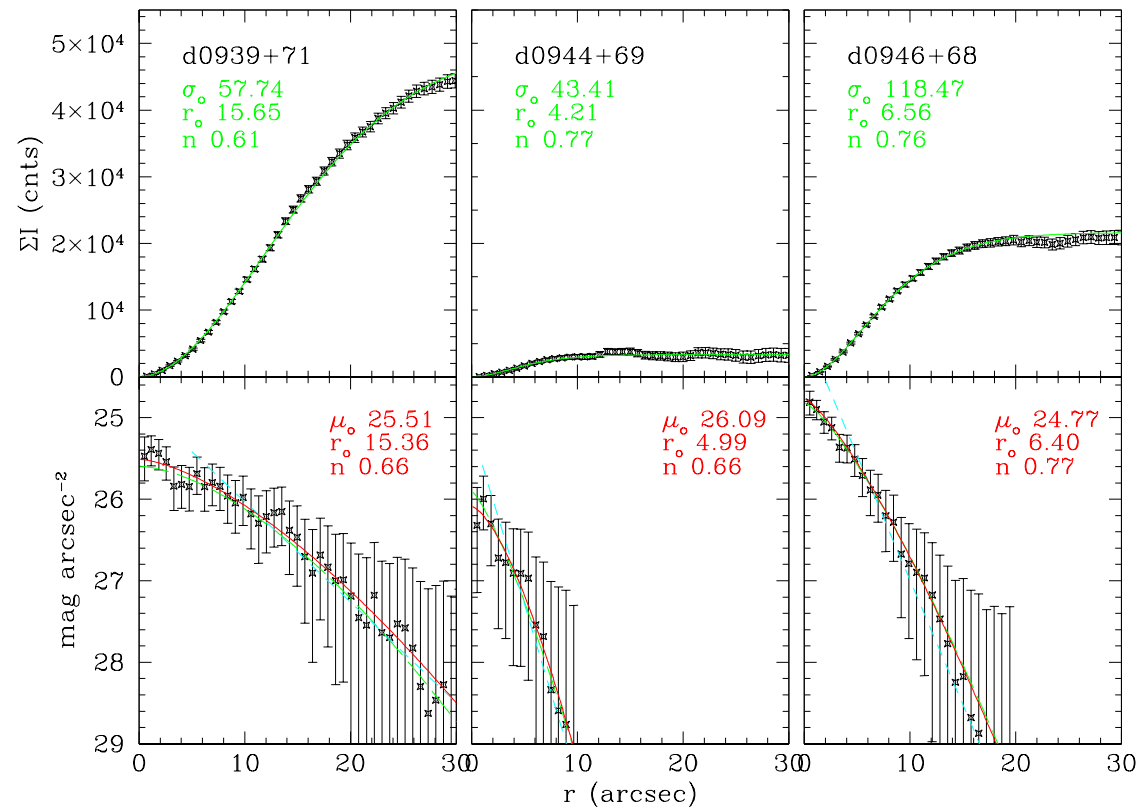

Fig. 23.- Fits for 3 potential background galaxies, as in Figure 20. 


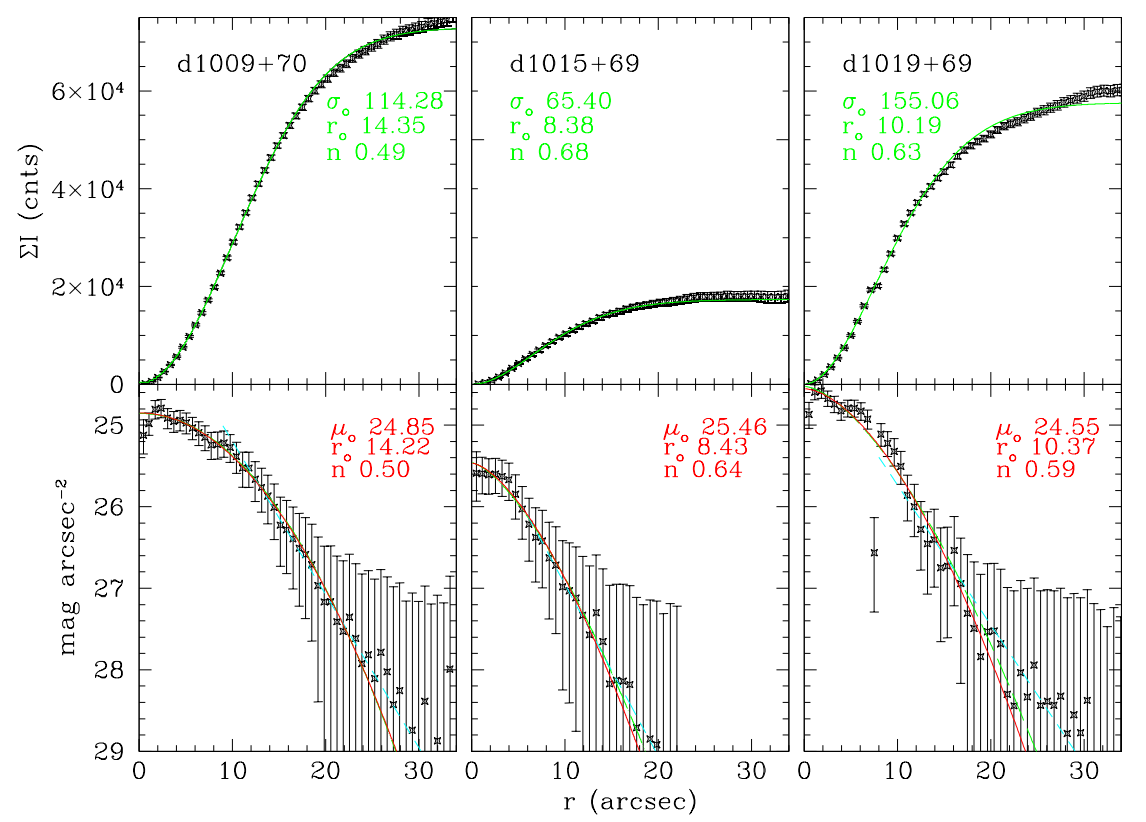

Fig. 24.- Fits for a further 3 likely background objects, as in Figure 20.

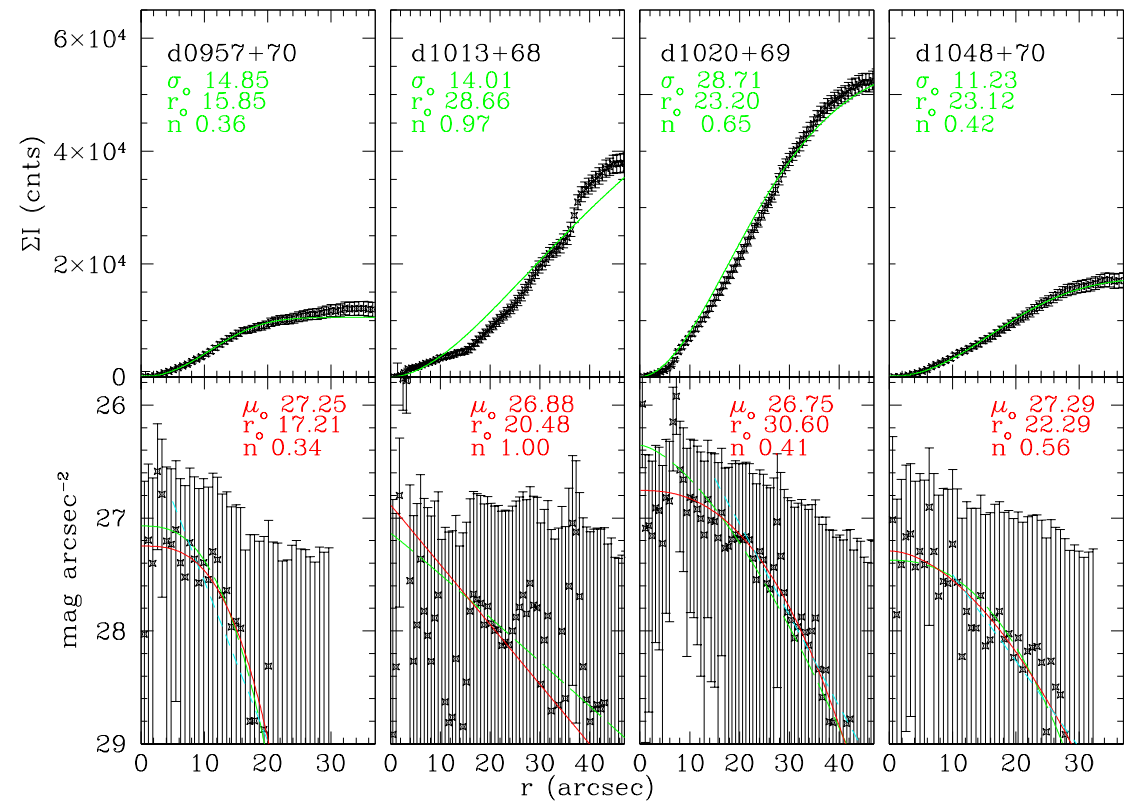

Fig. 25. - Fits for 4 of our poorer candidates, as in Figure 20. 


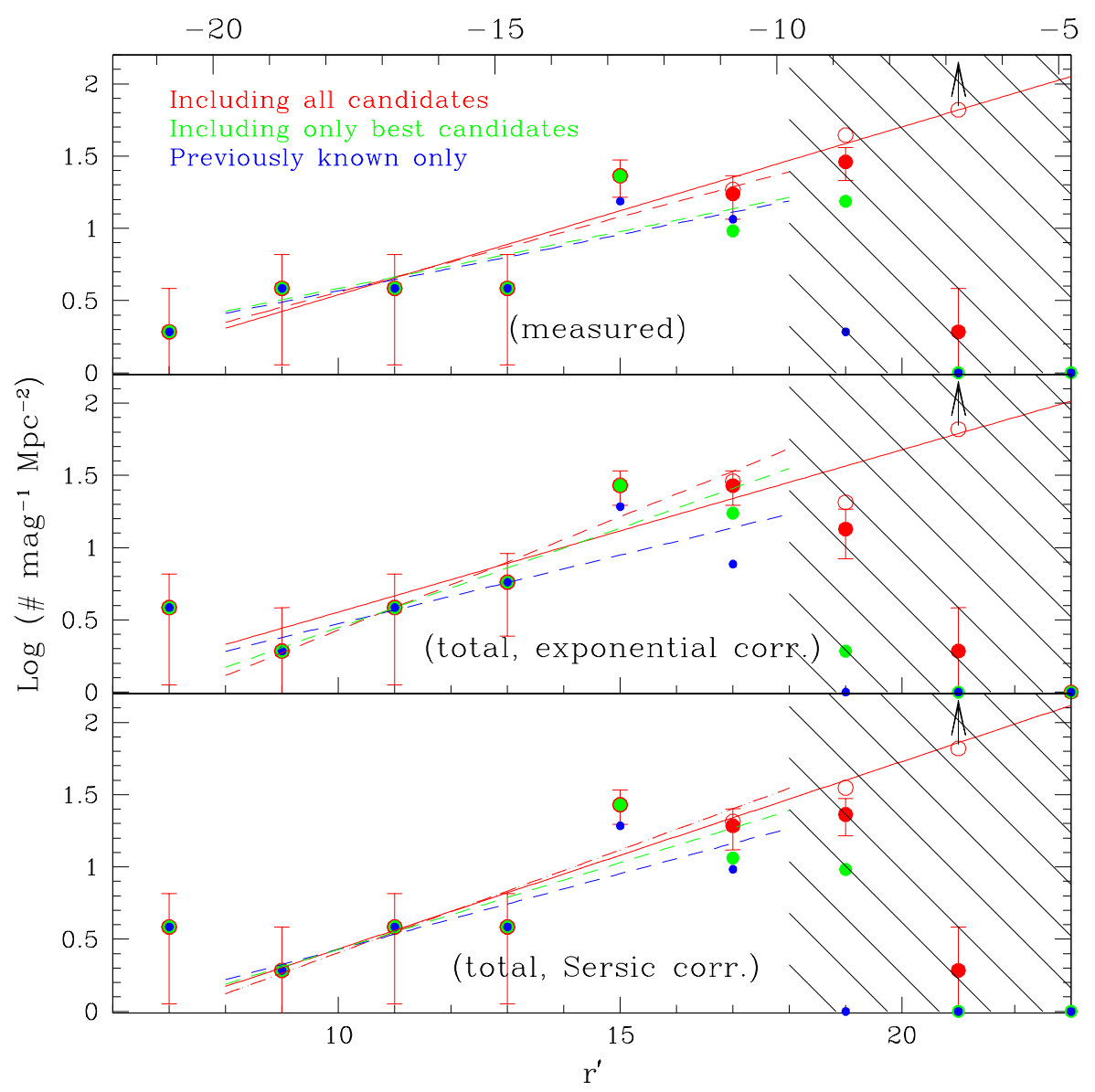

Fig. 26. - Differential luminosity function for the M81 group, using measured magnitudes (top), total magnitudes corrected assuming exponential profiles (middle), and total magnitudes assuming Sersic profiles (bottom). Counts are normalized to the area of the survey coverage. Solid red points include previously known group members and all candidates from this survey. Open circles are corrected for incompleteness. Green points include only the best candidates from this work. Blue points represent only previously known group members. The hatched region denotes where this survey is incomplete; brightward of this we expect nearly $100 \%$ completeness in the detection of member dwarfs. The best power law fits to the data brightward of our completeness limit are shown by the dashed lines, while the solid red line is the best fit for corrected counts. The arrow in the final datapoint signifies that a minimum completeness correction based on good seeing/low extinction simulations was applied. 


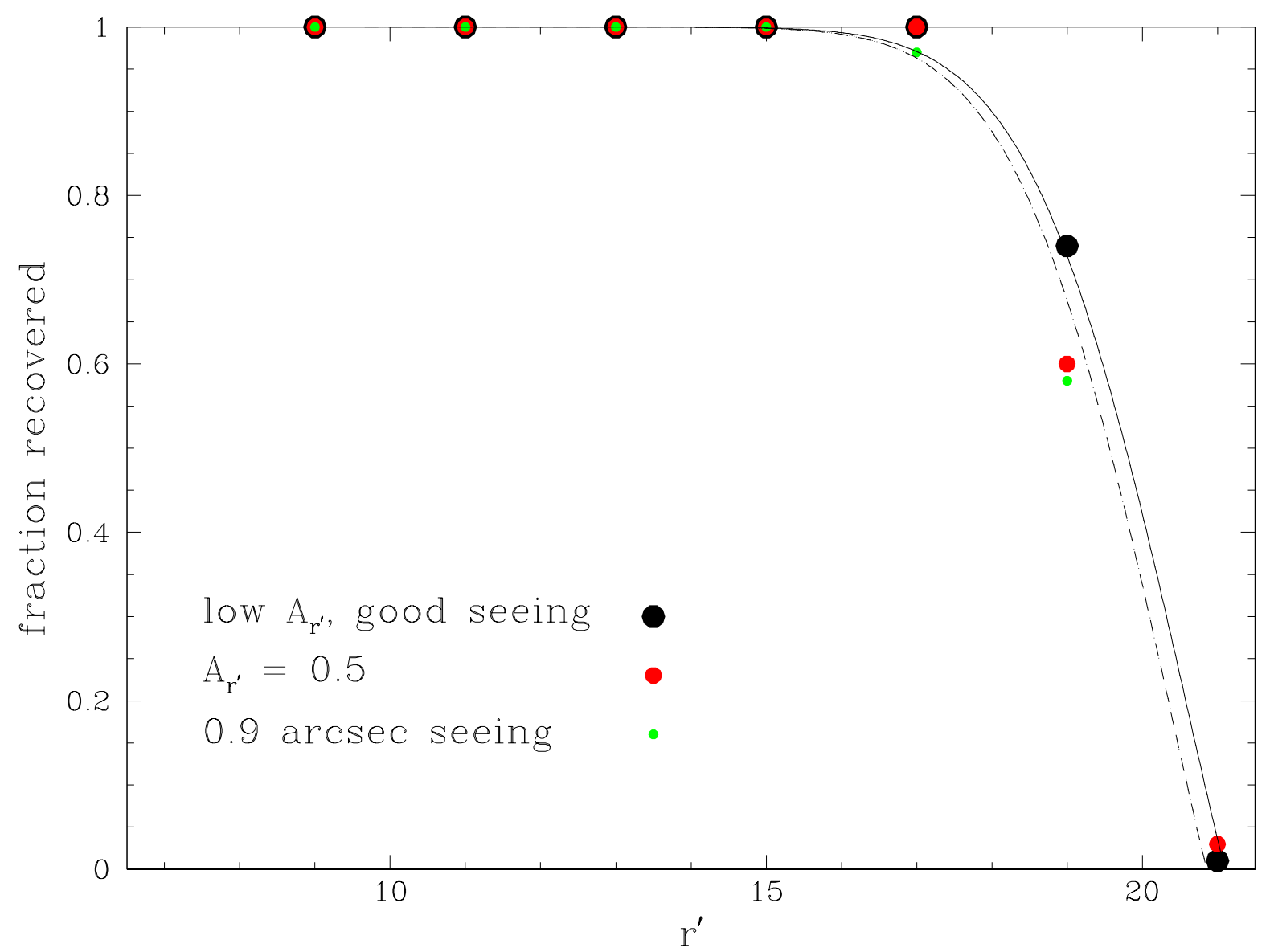

Fig. 27. - We plot detection efficiency as a function of magnitude. The solid line is the best fit to the good seeing, low extinction case, while the dashed line incorporates the survey average effects of seeing, extinction, single exposure regions, and loss of survey area due to coverag by brighter objects. 


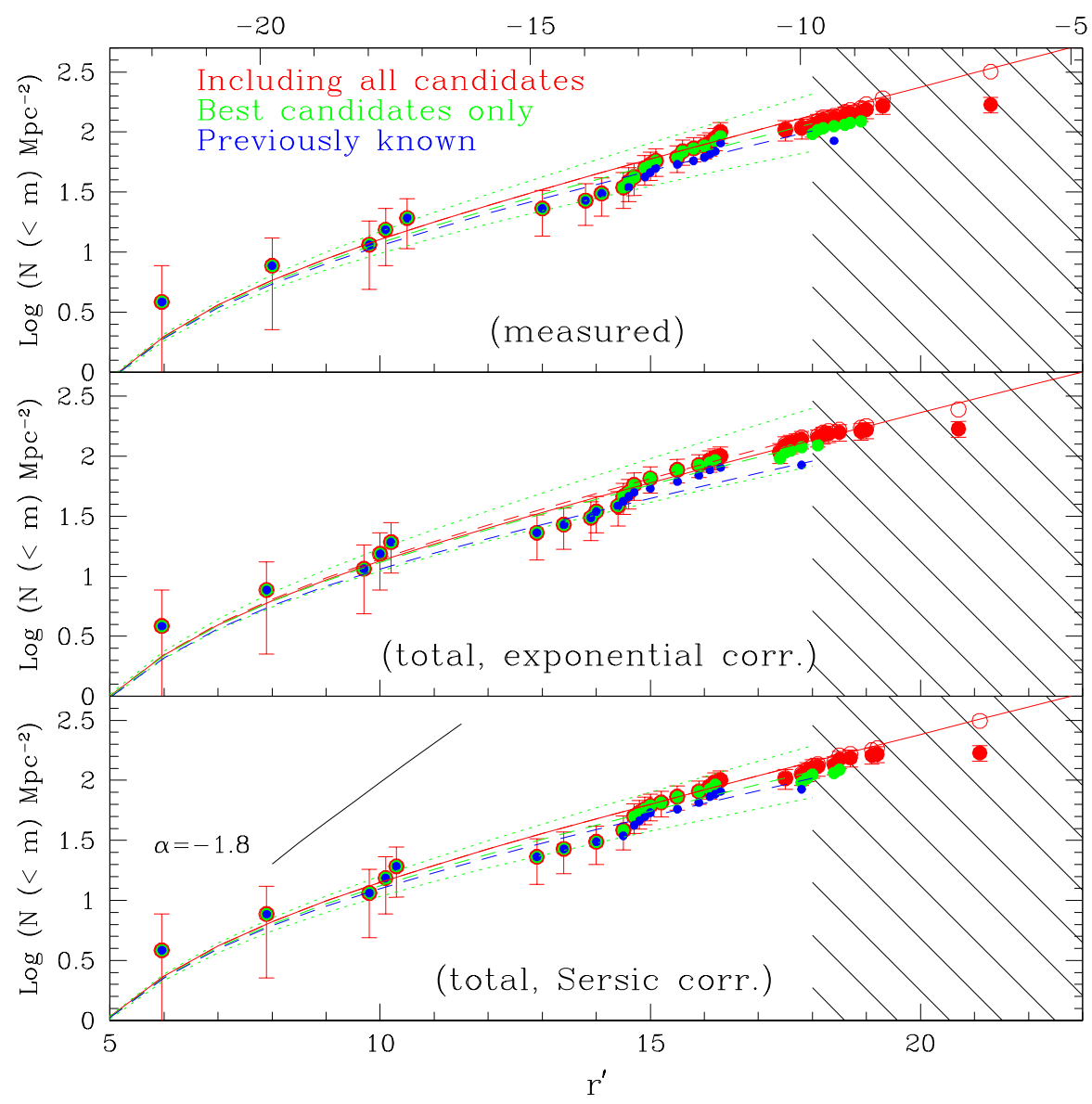

Fig. 28. - Cumulative luminosity function for the M81 group, using measured magnitudes (top), magnitudes corrected assuming exponential profiles (middle), and corrected magnitudes assuming Sersic profiles (bottom). Counts are normalized to the area of the survey coverage. Solid red points include previously known group members and all candidates from this survey. Open circles are corrected for incompleteness. The hatched region denotes where this survey is incomplete; brightward of this we expect nearly $100 \%$ completeness in the detection of member dwarfs. The best cumulative Schechter function fits to the data brightward of our completeness limit are shown by the dashed lines. The solid red line is the best fit to completeness corrected counts. Dotted lines are $\pm 1 \sigma$ fits for the sample of 'best' candidates. 


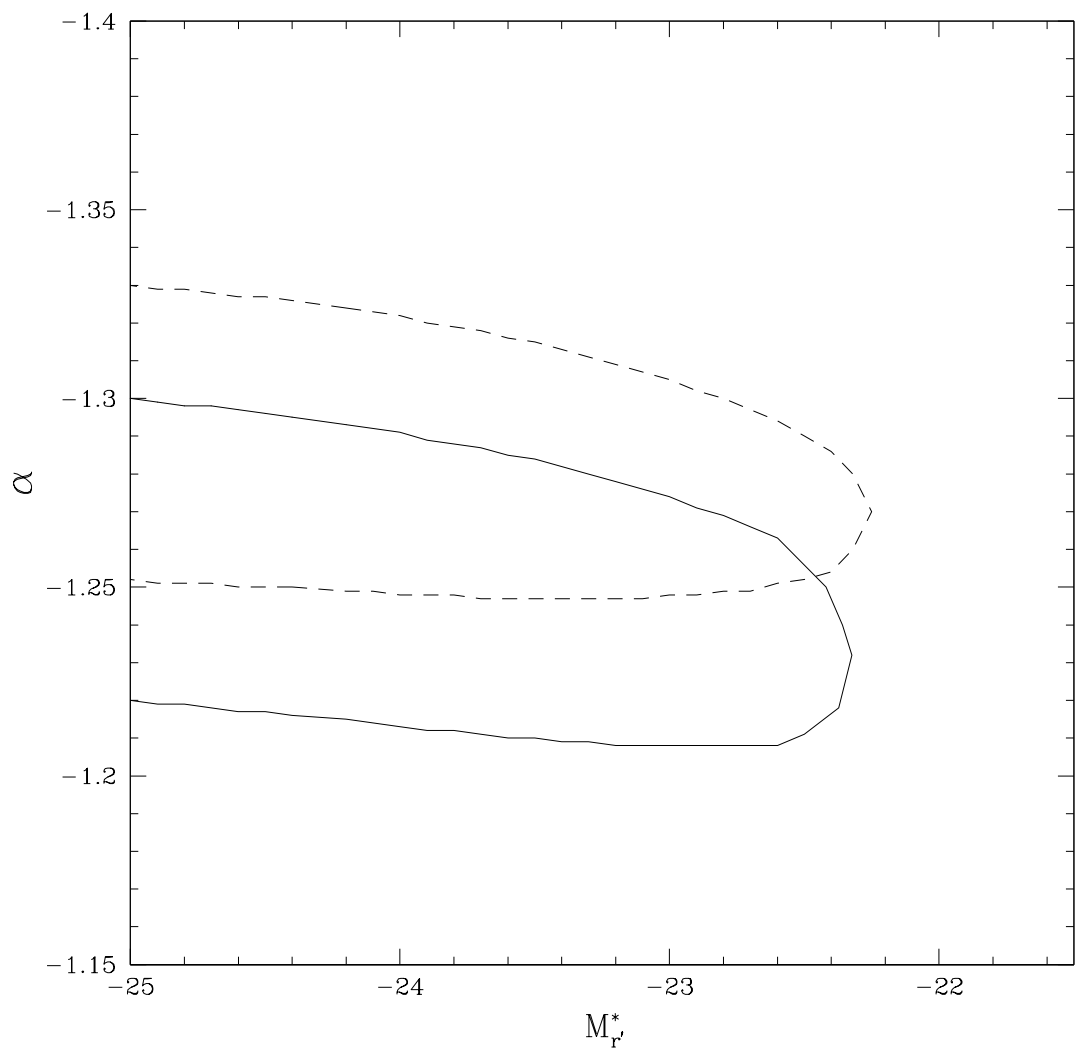

Fig. 29.- We display $1 \sigma$ error ellipses for the luminosity function parameters $\alpha$ and $M_{*}$ for our best (solid) and all (dashed) candidate samples using Sersic corrected magnitudes. The values for $M_{*}$ are unconstrained at the bright end. 


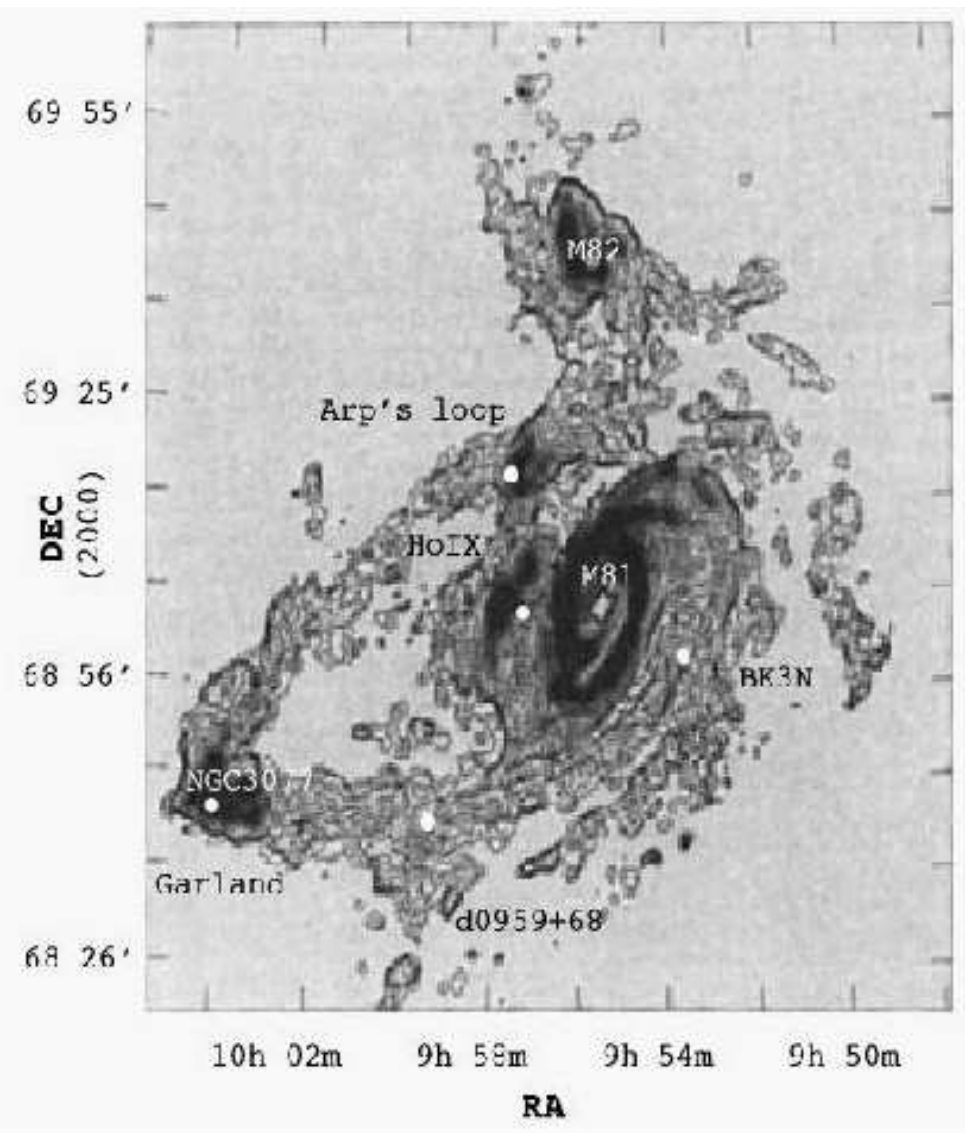

Fig. 30.- We display the M81 HI map of Yun et al. (1994). Overlaid are the positions of the five suspected tidal dwarfs. d0959+68 is found to lie in the tidal bridge between M81 and NGC3077. Adapted by permission of Macmillan Publishers Ltd: Nature, copyright 1994. 


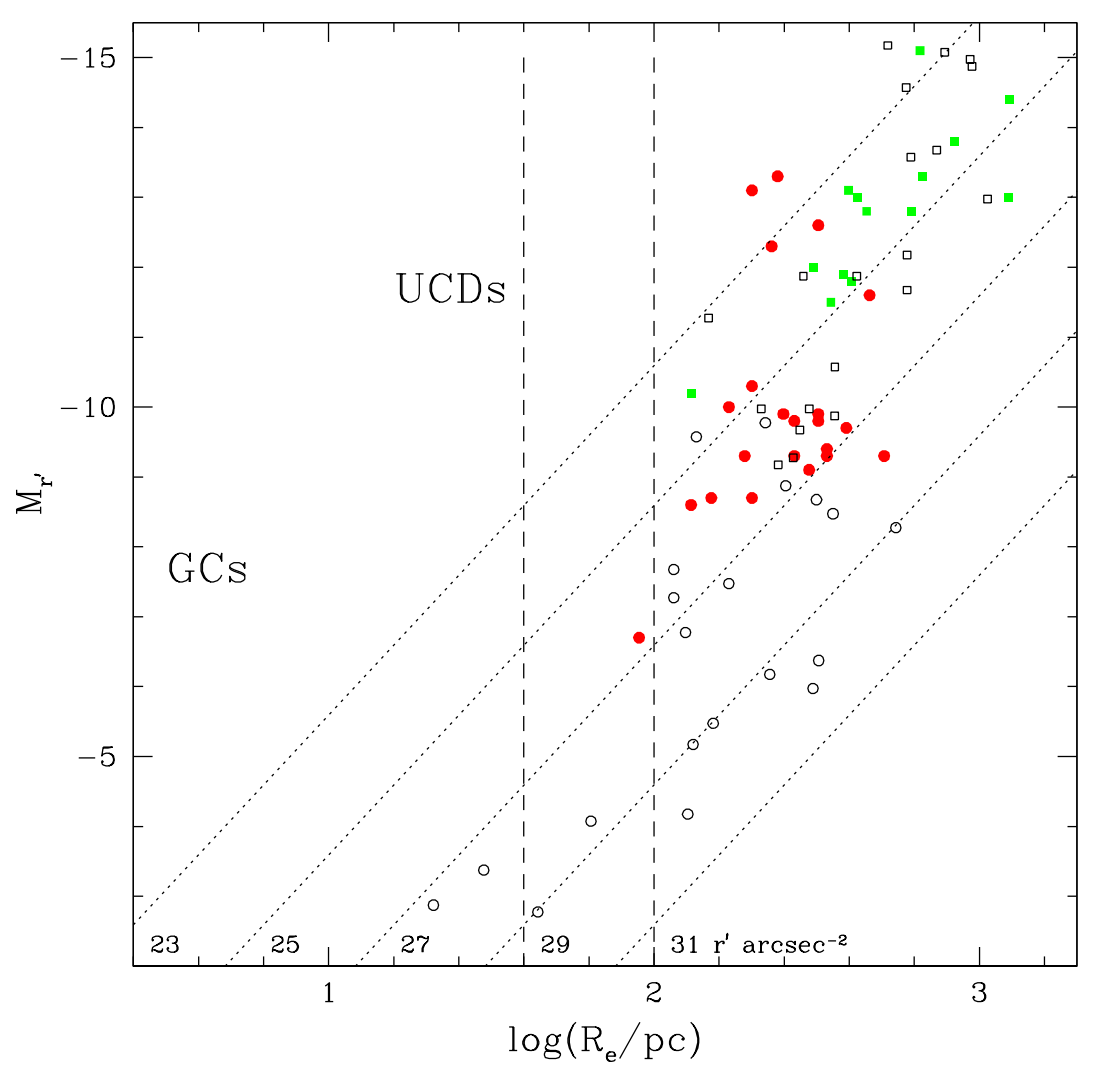

Fig. 31. - Locations of our M81 candidates on a total magitude vs half-light radius plane (filled circles), along with previously known M81 galaxies (filled squares), Local Group previously known galaxies (open squares) (Irwin \& Hatzidimitriou 1995; Mateo 1998; McConnachie \& Irwin 2006) and recent discoveries (open circles) (Simon \& Geha 2007; Belokurov et al. 2007a; Willman et al. 2005; Walsh et al. 2007; Zucker et al. 2006; Martin et al. 2006; Ibata et al. 2007; Irwin et al. 2008). To transform $V$ magnitudes to $r^{\prime}$, we assume $r^{\prime}=V-0.84(V-R)+0.13$ (Fukugita et al. 1996b). The rough locations of globular clusters and Fornax UCDs on this plane are shown. Globular Clusters have sizes that range up to $\sim 40 \mathrm{pc}$, while Local Group galaxies have sizes generally larger than $\sim 100$ pc. The size gap region falls between the two dashed lines. Lines of constant effective surface brightness are also shown. 


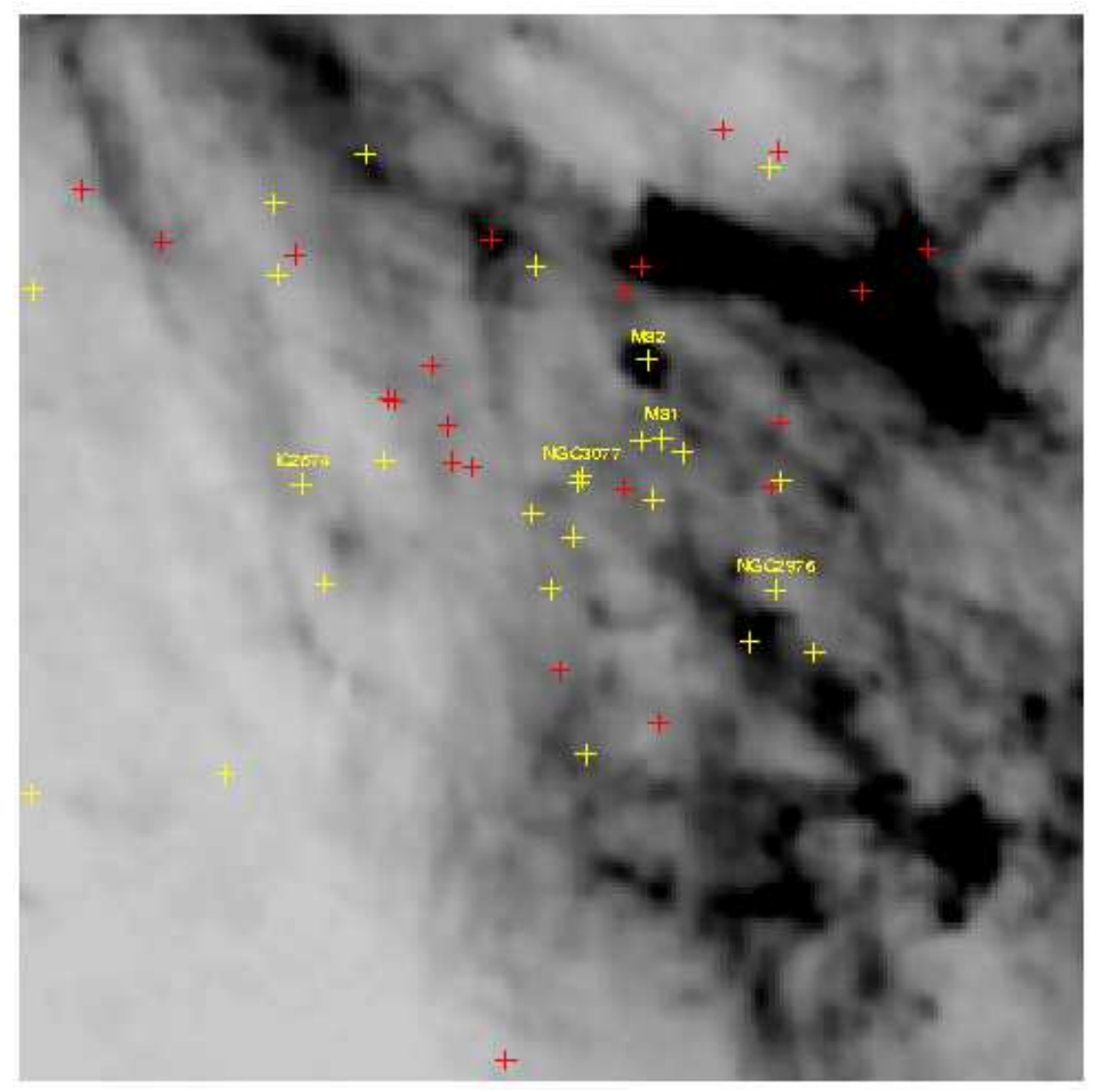

Fig. 32.- We display the M81 galaxy detections superimposed on the Schlegel et al. (1998) dust map. Red crosses represent newly discovered candidate group members while yellow crosses correspond to previously known group members. 


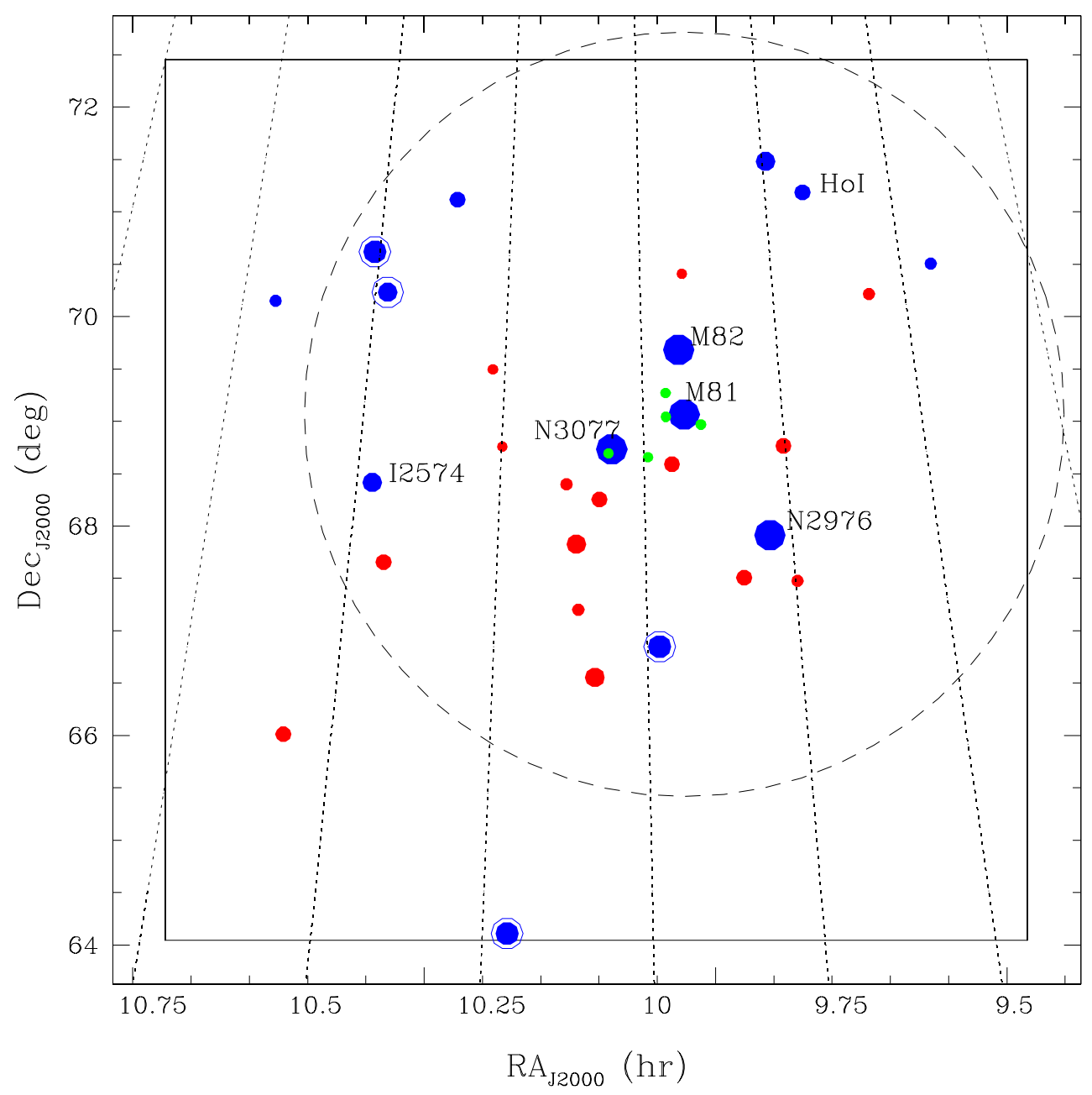

Fig. 33. - Projected distribution of early type (red) and late type (blue) galaxies. Green points indicate candidate tidal dwarfs. The location of BCD candidates, including DDO 82 , are marked by blue rings. We include in this plot only previously known and best candidates. Size indicates surface brightness with larger points denoting brighter effective surface brightness. 


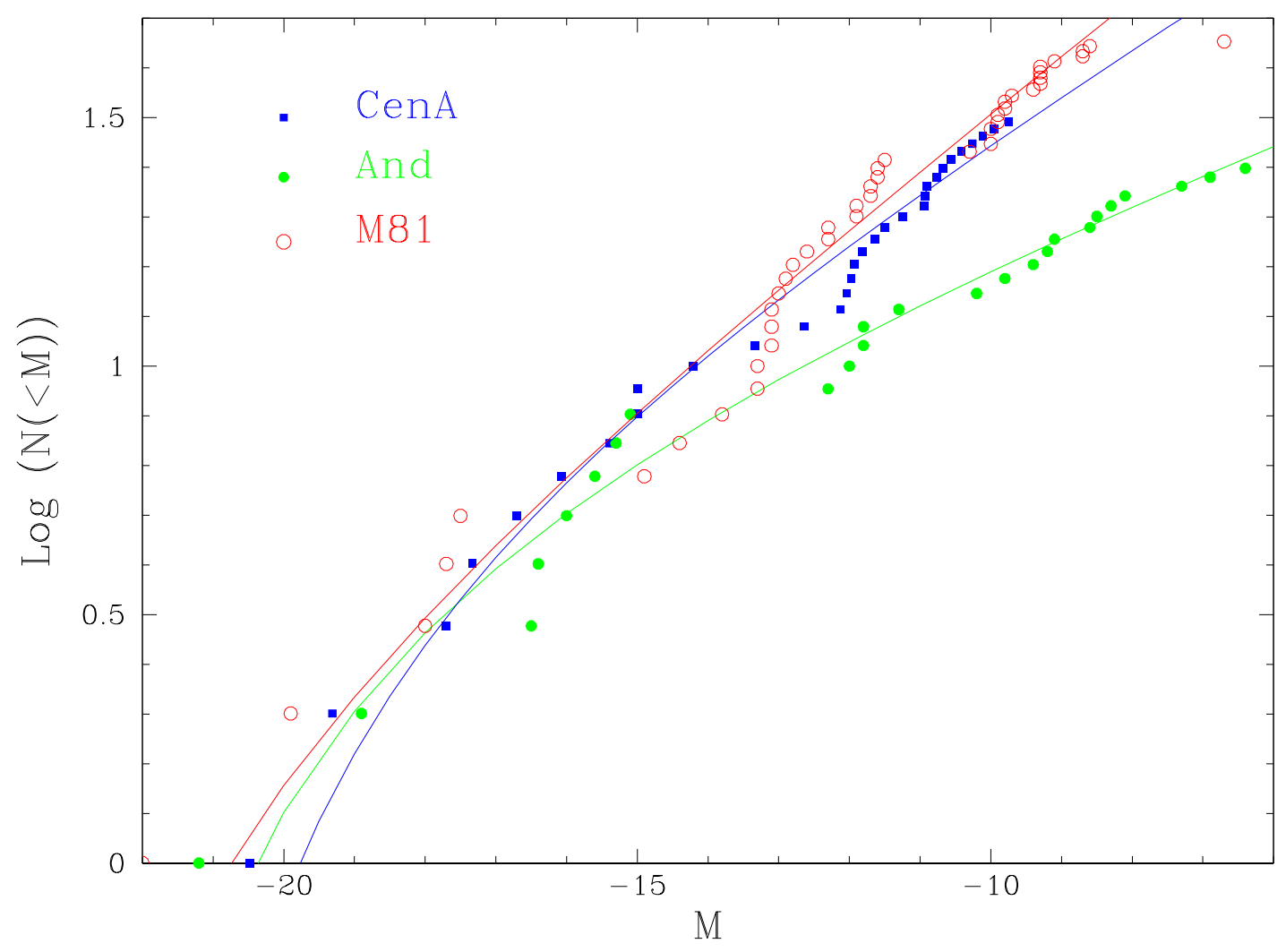

Fig. 34.- Cumulative LFs for Cen A ( $B$ band, squares), Andromeda ( $V$ band, solid circles), and M81 ( $r^{\prime}$ band, open circles) satellites. For clarity, we do not include error bars. Applying a cumulative Schechter function to these data, we find faint-end slopes of $-1.12_{-0.06}^{+0.06}$ for Andromeda systems, $-1.22 \pm_{-0.10}^{+0.04}$ for Cen A, and $-1.28_{-0.06}^{+0.06}$ for M81. 